\title{
UN MODELO DISCRETO PARA LA MECÁNICA CUÁNTICA RELATIVISTA
}

\author{
TESIS QUE PRESENTA \\ RAFAEL GONZÁLEZ CAMPOS \\ PARA LA OBTENCIÓN DEL GRADO DE \\ DOCTOR EN CIENCIAS
}

\begin{abstract}
MAYO DE 2000
UNIVERSIDAD AUTÓNOMA METROPOLITANA-IZTAPALAPA DIVISIÓN DE CIENCIAS BÁSICAS E INGENIERÍA
\end{abstract}




\section{AGRADECIMIENTO}

A mi amigo, el Dr. Luis Octavio Pimentel Rico

director de esta tesis,

por su guía y confianza en este proyecto. 


\section{Índice General}

1 Introducción $\quad 5$

2 Discretización de las reglas cuánticas $\quad 9$

2.1 Discretización en una variable . . . . . . . . . . . . . . . . . 9

2.2 Discretización en varias variables . . . . . . . . . . . . . 15

3 El impulso angular en un espacio discreto 22

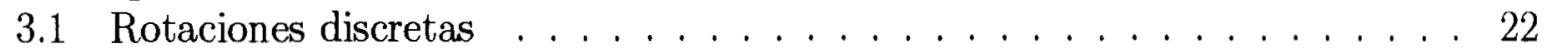

3.1 .1 Caso impar . . . . . . . . . . . . . . . . . . 24

3.1 .2 Caso par . . . . . . . . . . . . . . . . . . 26

3.2 Una matriz finita para $L^{2} \ldots \ldots \ldots \ldots \ldots \ldots \ldots$

3.3 Otra matriz para $L^{2} \ldots \ldots \ldots \ldots \ldots \ldots \ldots$

3.4 Las relaciones de conmutación . . . . . . . . . . . . . . . . . 31

4 La ecuación de Schrödinger discreta $\quad 35$

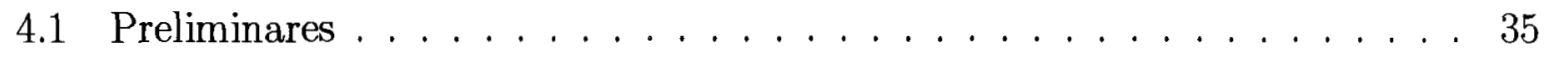

4.2 El átomo de Hidrógeno . . . . . . . . . . . . . . . . . . . . . . . . 36

4.3 Impulso angular total . . . . . . . . . . . . . . . . . . . . . 40

4.4 Estructura fina del Hidrógeno . . . . . . . . . . . . . . . . . . . 41

4.5 El átomo de Hidrógeno en un campo magnético . . . . . . . . . . . . 43

5 Ecuaciones relativistas discretas $\quad 49$

5.1 Preliminares . . . . . . . . . . . . . . . . . . . . . . . . . . . . . 49

$5.2 \quad$ La ecuación de Klein-Gordon . . . . . . . . . . . . . . . . . . . . . . . . . 49

5.2 .1 Ecuación de continuidad . . . . . . . . . . . . . . 55

5.2 .2 Una raíz de la ecuación de Klein-Gordon discreta . . . . . . . . . 57

5.3 La ecuación de Dirac . . . . . . . . . . . . . . . . . 58

6 Conclusiones $\quad 66$

$\begin{array}{ll}\text { A Producto de Kronecker } & 68\end{array}$

B Derivadas parciales discretas $\quad 72$ 
C Convergencia del esquema de proyección 


\section{Capítulo 1}

\section{Introducción}

Usualmente se supone en la Física que el espacio-tiempo es de naturaleza continua. Si bien la Mecánica Cuántica ha liberado a la Física de la restricción de continuidad sobre algunas de sus observables, la idea de continuidad en el espacio y en el tiempo se mantiene por consenso. Sin embargo, esto no ha impedido que desde los inicios de la Mecánica Cuántica se investigue en direcciones diferentes con la esperanza de eliminar ciertas divergencias (y sus renormalizaciones) que ocurren en la teoría cuántica de campos. A grandes rasgos, es posible clasificar en dos grandes grupos el trabajo publicado sobre este problema: uno, que trata de obtener una discretización del espacio-tiempo a partir de primeros principios, es decir, mediante la definición de operadores concernientes con las variables correspondientes y otro, que parte en principio de una malla (lattice) del espacio-tiempo y desarrollan una teoría discreta en estos términos (dentro de este grupo podemos situar al presente trabajo). Snyder [48], Pauli [44], Wigner [53], Yukawa [55], Bergmann y Brunning [3], Pirani y Schild [45], Dirac [21] y de Witt y De Witt [54], se cuentan entre los pioneros que estudiaron directa o indirectamente el problema de la cuantización del espacio-tiempo y abrieron nuevos problemas relacionados al tema. Si bien es frecuente encontrar en la literatura que las referencias a los primeros trabajos sobre cuantización del espacio-tiempo comienzan en [48], en realidad hay trabajos anteriores al de Snyder: en [51] se encuentra una referencia a M. Markov, Journ. of Phys. USSR 2 (1940) 453, en [47] se encuentran citados otros artículos anteriores y en [29] se encuentra una extensa bibliografía (más de 300 referencias) sobre el problema general de la estructura del espacio-tiempo a pequeña escala (que engloba a la discretización) y las diferentes maneras de manejarlo, así como las conexiones que hay entre las distintas técnicas de acercamiento al problema.

Snyder da al tiempo (y a la posición) el carácter de operador y supone que los espectros de estos operadores (no necesariamente continuos) son invariantes de Lorentz y obtiene que para la posición, éstos consisten en un conjunto de valores discretos múltiplos de un valor fundamental (cuanto de longitud) mientras que para el tiempo obtiene un espectro continuo al igual que para el impulso. Pauli establece una equivalencia entre las variables de este problema y las coordenadas en un espacio de De Sitter. Un hecho importante que se destaca en el trabajo de Snyder, es que los conmutadores de variables conjugadas 
en el espacio-tiempo no conmutan canónicamente, hecho que no se contrapone con las ecuaciones de movimiento, como Wigner muestra en [53].

A partir de entonces la literatura científica sobre este problema se ha visto engrosada por muchas otras contribuciones (ver [29]) de las que sólo referimos algunas más [51], [26], [33], [39], [6], [27], [7], [28], y [52]. Entre éstas, llama la atención que se ha tratado de determinar una cota para el posible cuanto de longitud en base a resultados experimentales [6].

A pesar de la gran cantidad de trabajo en esta dirección, aún no ha sido posible desarrollar una teoría discreta adecuada. Estos modelos presentan siempre algún aspecto que hace difícil que la teoría subyacente se acepte en general, por ejemplo, el trabajo de Snyder y otros ([48], [7]) predice un espacio discreto, un cuanto de longitud, pero un impulso y tiempo continuos y esto rompe la reciprocidad que se espera en las variables. Además, como ya lo dijimos, predice para la posición eigenvalores múltiplos de una unidad fundamental que si bien se encuentra dentro de la posibilidad de que sea así (ver [28]), da lugar a que la estructura del espacio sea regular (lattice), lo cual es un caso particular de otro tipo de estructuras más complejas, posibles y útiles; sobre todo considerando la posibilidad de un modelo para la gravedad cuántica. Otros acercamientos al problema ([39], [27]) consideran puntos de espacio-tiempo no necesariamente equiespaciados, pero además de partir de las leyes establecidas y tomar a priori como aproximación los resultados en su modelo discreto (obtenidos por simple colocación y aproximación de integrales por sumas) recurren a un promedio (ver también [26]) sobre las distancias debido a la complejidad del análisis para hacer cálculos, sin que la manera de seleccionar los puntos discretos quede clara. Más recientemente han aparecido otros acercamientos al problema, ver por ejemplo [33] y [52] produciendo discretizaciones del espacio y de los impulsos libres de los problemas inherentes al modelo de Snyder en mallas irregulares, pero que dejan a un lado el problema de discretización temporal. En [36] y [35] se usa una malla regular en el plano $x--t$ para discretizar la ecuación de Dirac y obtener que la solución de la ecuación discreta puede reescirbirse como el propagador que se obtiene en en modelo de ajedrez de Feynman [25]. Sobre otros modelos discretos de espacio-tiempo y otros acercamientos al problema puede consultarse la revisión referida previamente [29] que da un panorama general del problema y de los intentos para resolverlo.

Así, la falta de un consenso sobre la discretización del espacio-tiempo permite proponer otros caminos y hace deseable disponer de un modelo de discretización tanto en la posición como el tiempo, libre de las dificultades ya mencionadas.

En trabajos desarrollados desde hace una decena de años ([13], [14] y [15]), se ha intentado establecer las bases de una formulación de la Mecánica Cuántica ordinaria en espacios lineales de dimensión finita, proyectando ortogonalmente los operadores correspondientes a un conjunto completo de observables compatibles sobre alguna base (truncada) de funciones del espacio de estados. Así, al proyectar dichos operadores sobre estos subespacios de dimensión finita y reconstruír los restantes siguiendo las relaciones entre éstos y los del conjunto completo compatible, se obtiene un espectro discreto para cada uno. Ya que la proyección no toma en cuenta a todas las componentes del espacio de estados, es de esperarse que esta técnica reproduzca en forma aproximada las soluciones de problemas 
cuánticos continuos y en este sentido puede tomarse como un método de aproximación. La aproximación lograda puede ser numérica o analítica, según se resuelva el problema finito mediante métodos computacionales o con métodos de la teoría de la aproximación. En este punto podría parecer que esta técnica de discretización fuera incapaz de aproximar soluciones cuyo espectro sea continuo, ya que genera matrices de dimensión finita como representaciones de las observables cúanticas, sin embargo, a lo largo de este trabajo tendremos ocasión de mostrar que esto no es así, es decir, que el espectro de estas matrices pueden aproximar al espectro continuo que tienen algunas observables, y que esta técnica es más que un método numérico ya que las relaciones de conmutación entre la posición y el impulso y las del impulso angular adoptan en el espacio de proyección las formas canónicas bien conocidas siempre que se restrinja en cierta forma el dominio de aplicación de los operadores discretos, afianzando así la construcción de una formulación de la Mecánica Cuántica en espacios lineales de dimensión finita. Sin embargo, no es tanto en la Mecánica Cuántica ordinaria donde se deben investigar las consecuencias de esta formulación como en la Teoría Cuántica Relativista, por ser ésta una teoría más completa, sobre todo si parte del interés a futuro es tener un acercamiento a Gravedad Cuántica, ya que como se menciona en [29], es sólo cuando se trata de incluír gravedad en Teoría Cuántica de Campos que se encuentra una razón sólida para creer en un espacio-tiempo discreto. Sin una motivación física, esta formulación no pasa de ser una curiosidad matemática.

Como ejemplo de que este método no sólo obtiene resultados numéricos, sino que puede dar explicaciones alternativas de ciertos fenómenos en algunos problemas cuánticos podemos citar la referencia [15], en donde la aparente doble solución para las eigenfunciones del hamiltoniano del oscilador para-Bose obtenidas por Ohnuki y Kamefuchi ([43]) se explica como la dependencia de la única solución en la paridad (par o impar) del número que define la dimensión del espacio de proyección, sin recurrir a las reglas de superselección dadas en ([43]) o a la separación del dominio del operador de impulso dada en [42].

El propósito del presente trabajo ya lo hemos dejado entrever en los últimos párrafos: presentar una técnica de proyección para la mecánica cuántica ordinaria y para la relativista, que produce representaciones matriciales de dimensión finita para las observables cuánticas, valores discretos para las coordenadas del espacio-tiempo y ecuaciones matriciales a resolver para las ecuaciones de onda, y que mantiene (restringiendo el dominio de aplicación) las formas canónicas de conmutación entre las componentes de la posición y del impulso lineal, así como las correspondientes a las componentes del impulso angular. Dividiremos en tres partes este trabajo. En la primera (Capítulos 2 y 3) daremos un resumen de la técnica de discretización para problemas unidimensionales lineales o angulares. Discutiremos la aplicación de una transformada de Fourier discreta y extenderemos el método a problemas multidimensionales, particularmente en coordenadas cartesianas y esféricas. Mostraremos que las representaciones de las componentes del impulso lineal y angular en el espacio discreto conservan las mismas relaciones a las derivadas parciales discretas que tienen en el caso continuo. Así mismo mostraremos que los conmutadores de estas componentes también mantienen las formas usuales en ciertos subespacios del espacio de proyección. En el Capítulo 4 trataremos el importante caso del átomo de 
Hidrógeno dentro de este contexto de discretización con dos subcasos. En uno trataremos el problema de la estructura fina del Hidrógeno y aplicaremos el método de discretización como un método numérico de solución. El otro caso será el problema de un campo magnético uniforme de intensidad arbitraria. Finalmente, en el Capítulo 5 estudiaremos las ecuaciones de Klein-Gordon y de Dirac dentro de este contexto discreto y obtendremos sus propagadores. La solución discreta de la ecuación de Dirac podremos reescribirla en la forma dada por el "modelo de ajedrez" de Feynman descrito en su trabajo sobre integrales de trayectoria [25]. 


\section{Capítulo 2}

\section{Discretización de las reglas cuánticas}

\subsection{Discretización en una variable}

En esta sección haremos un resumen del método en que nos basaremos para discretizar las ecuaciones de onda y, de manera indirecta, al espacio y al tiempo. Este método puede verse como una proyección ortogonal del conjunto de funciones a que pertenece la solución de una ecuación diferencial con ciertas condiciones a la frontera dadas, sobre subespacios de funciones más simples (método de Galerkin). Las bases de esta técnica son fundamentalmente dos (ver por ejemplo [8]):

1. La suposición de que la función de onda $\Psi(x)$ puede escribirse o aproximarse en la forma $\Psi(x)=\gamma(x) f(x)$, donde $\gamma(x)$ es una función que lleva las condiciones a la frontera así como la parte no lisa de la solución, y $f(x)$ es un polinomio. Como ejemplos, tenemos al oscilador armónico y al átomo de hidrógeno entre otros.

2. El uso de una representación matricial finita de la derivada $d / d x$ obtenida (primeramente por $\mathrm{F}$. Calogero y M. Bruschi [9]) a través de interpolación lagrangiana y que produce resultados exactos para funciones polinomiales.

Este método ha sido aplicado a problemas de valores a la frontera singulares y no lineales en una dimensión [19], produciendo soluciones convergentes con un número reducido de nodos y con una fórmula aproximada para el error.

Consideremos la ecuación de Schrödinger

$$
-\Psi^{\prime \prime}(x)+2 V(x) \Psi(x)=2 E \Psi(x), \quad a<x<b,
$$

escrita en algún sistema de unidades adecuado y con las condiciones usuales $\Psi(a)=$ $\Psi(b)=0$. La substitución

$$
\Psi(x)=\gamma(x) f(x)
$$

en (2.1) produce la ecuación diferencial

$$
-f^{\prime \prime}(x)-r(x) f^{\prime}(x)+q(x) f(x)=2 E f(x)
$$


donde $r(x)=2 \gamma^{\prime}(x) / \gamma(x)$ y $q(x)=2 V(x)-\gamma^{\prime \prime}(x) / \gamma(x)$. Obsérvese que el potencial $V(x)$ determina el tipo de singularidad existente en alguno (o ambos) de los extremos del intervalo $(a, b)$ para el problema diferencial $(2.3)$ y esto a su vez determina el tipo de función $\gamma(x)$ a usar (piénsese por ejemplo en una singularidad regular y las potencias características). Es decir, el potencial determina en parte a la función $\gamma(x)$. En la medida en que $f(x)$ pueda aproximarse por un polinomio, los vectores $N$-dimensionales $f^{(k)}$, $f^{(0)} \equiv f$, formados con las $k$-ésimas derivadas de $f(x)$ evaluadas en los nodos, i.e, de elementos $f^{(k)}\left(x_{j}\right)$ (con $\left.k<N\right)$, pueden escribirse como

$$
f^{(k)}=\left(\tilde{D}_{x}\right)^{k} f+e_{f}^{k},
$$

donde $\tilde{D_{x}}$ es la matriz $N \times N$

$$
\tilde{D}_{x}=Q D_{x} Q^{-1}, \quad D_{x}=D_{x}^{0}+d
$$

con

$$
\left(D_{x}^{0}\right)_{i j}=\left\{\begin{array}{ll}
0, & i=j, \\
\frac{1}{\left(x_{i}-x_{j}\right)}, & i \neq j,
\end{array} \quad d_{i j}=\delta_{i j} \sum_{l=1}^{N} \frac{1}{\left(x_{i}-x_{l}\right)}, \quad i, j=1,2, \cdots, N,\right.
$$

y $e_{f}^{k}$ es el error asociado a $f^{(k)}(x)$. Aquí, $\Sigma^{\prime}$ siginifica la suma sobre $l \neq i$ y $Q$ es una matriz diagonal que lleva a lo largo de su diagonal principal las derivadas $p^{\prime}\left(x_{j}\right)$ del polinomio

$$
p(x)=\prod_{k=1}^{N}\left(x-x_{k}\right) .
$$

Si $f(x)$ es un elemento de $\pi_{N-1}(x)$, el espacio de polinomios de grado $N-1$ a lo más, la fórmula (2.4) tiene error cero, es decir, produce resultados exactos, ya que la forma funcional de cualquier polinomio perteneciente a $\pi_{N-1}(x)$ puede recobrarse a partir de una $N$-ada de sus valores a través de una interpolación lagrangiana (ver [10]). Esto significa que cualquier problema diferencial cerrado en $\pi_{N-1}(x)$, puede resolverse también como un problema matricial de dimensión finita produciendo la misma solución.

Si $f(x)$ no es un polinomio, pero es suficientemente diferenciable (para escribirse en una serie de Taylor, por ejemplo), la substitución de $\tilde{D}_{x}$ por $d / d x$ en (2.3), producirá resultados convergentes en la medida que los coeficientes $c_{n}$ de la serie de Taylor que defina a $f(x)$ se aproximen a cero para $n$ grande (para que $f(x)$ pueda aproximarse por un polinomio). En esto radica el carácter de proyección de este método y en el apéndice $\mathrm{C}$ se da una demostración de la convergencia de este esquema de derivación a $N$ puntos.

Para obtener la forma discreta de (2.3), se substituye $d / d x$ por $\tilde{D_{x}}$ y la variable $x$ por $X$, la matriz diagonal que lleva los puntos $x_{j}, j=1,2, \cdots, N$, a lo largo de su diagonal principal, para obtener

$$
\left[-\left(\tilde{D}_{x}\right)^{2}-R \tilde{D}_{x}+Q\right] f^{*}=2 E^{*} f^{*}
$$

donde $E^{*}$ y $f^{*}$ son aproximaciones al eigenvalor exacto $E$ y al vector cuyos elementos son los valores exactos de $f(x)$ en los nodos. $R$ y $Q$ son matrices diagonales cuyas diagonales 
principales llevan los valores $r\left(x_{j}\right)$ y $q\left(x_{j}\right), j=1,2, \cdots, N$, respectivamente. Para que este método de discretización pueda tener confiabilidad, debe proyectar un problema diferencial hermitiano a un problema matricial también hermitiano (o con eigenvalores reales). No es difícil mostrar que si se satisface la igualdad entre la diagonal de $\tilde{D}_{x}$ y la matriz $R$

$$
d=-\frac{1}{2} R
$$

la matriz de $(2.8),-\left(\tilde{D_{x}}\right)^{2}-R \tilde{D}_{x}+Q$ resulta ser simétrica (salvo por una transformación de similitud) y por lo tanto tiene eigenvalores reales. La ecuación (2.9) puede reescribirse en términos de $\gamma(x)$

$$
\sum_{l=1}^{N} \frac{1}{\left(x_{j}-x_{l}\right)}=-\frac{1}{2} r\left(x_{j}\right)=-\frac{\gamma^{\prime}\left(x_{j}\right)}{\gamma\left(x_{j}\right)}, \quad j=1,2, \cdots, N .
$$

Esta condición es en realidad un conjunto de $N$ ecuaciones no lineales cuya solución existe en $(a, b)$, y es única si $\gamma(a)=\gamma(b)=0$ y $\log \gamma(x)$ es una función cóncava (ver [46]). El intervalo que se esté considerando puede ser finito o infinito. Puesto que $\gamma(x)$ se anula en los extremos, $\Psi(x)$ satisface las condiciones requeridas por la Mecánica Cuántica. La solución de (2.10) consiste en un conjunto de nodos $x_{j}$, con $a<x_{1}<x_{2}<\cdots<x_{N}<b$ y cuya disposición en el eje real depende obviamente de la forma funcional de $\gamma(x)$. Ya que esta función incluye información del potencial a través de la ecuación diferencial, es el potencial el que determina la posición de los nodos en la recta. De manera análoga a la discretización de la energía, en donde interviene también el potencial, podemos interpretar a las diferencias de puntos adyacentes $\varepsilon_{j}=x_{j+1}-x_{j}$ como cuantos de longitud. En este sentido, el método presentado, aunque aparentemente numérico, puede interpretarse como un método de discretización espacial.

Es posible modificar esta técnica y hacerla un poco más simple. Consideremos que en (2.2) $f(x)$ es un polinomio de grado menor que $N$. Entonces

$$
\Psi^{\prime}\left(x_{j}\right)=\gamma\left(x_{j}\right)\left[f^{\prime}\left(x_{j}\right)+\left(\gamma^{\prime}\left(x_{j}\right) / \gamma\left(x_{j}\right)\right) f\left(x_{j}\right)\right] .
$$

Si denotamos por $\Psi^{\prime}, f^{\prime}$ y $f$ los vectores formados con los valores de las funciones correspondientes evaluadas en los nodos y tomamos en cuenta (2.5) esta expresión toma la forma

$$
\Psi^{\prime}=G\left(f^{\prime}-d f\right)=G Q D_{x}^{0} Q^{-1} f
$$

donde $d$ está dada por (2.6) y $G$ es una matriz diagonal con entradas $\gamma\left(x_{j}\right)$ a lo largo de su diagonal principal. Por lo tanto,

$$
\Psi^{\prime}=\tilde{D_{x}^{0}} \Psi
$$

donde

$$
\tilde{D}_{x}^{0}=S_{x} D_{x}^{0} S_{x}^{-1}
$$

y

$$
S_{x}=G Q
$$


Así vemos que la matriz antisimétrica $D_{x}^{0}$, construída con los nodos obtenidos a partir de (2.10) [salvo la transformación de similitud dada en (2.14)], es una representación matricial finita de la derivada $d / d x$ en el espacio de funciones de la forma (2.2), con $f(x)$ polinomio de grado menor que $N$. Esto conduce a proponer la simple substitución de $D_{x}^{0}$ directamente en (2.1), sin pasar por las substituciones (2.2) y (2.5), para obtener la ecuación matricial

$$
\left[-\left(D_{x}^{0}\right)^{2}+2 V(X)\right] \phi^{*}=2 E^{*} \phi^{*}
$$

donde la matriz diagonal $X$ es la representación finita de la variable $x$ que lleva en su diagonal principal los valores $x_{j}, j=1,2, \cdots, N$, y $V(X)$ es la matriz diagonal con elementos no nulos dados por $V\left(x_{j}\right) . E^{*}$ es en principio un aproximante a los eigenvalores de la energía $E$ y $\phi *$ es el eigenvector dado en términos del vector $\psi^{*}$ [cuyos elementos aproximan a $\Psi\left(x_{j}\right)$ ] por $\phi^{*}=S^{-1} \psi^{*}$, donde $S$ es la matriz de similitud dada en (2.14). Este método ha sido usado para resolver numéricamente el oscilador anarmónico y el oscilador para-Bose (ver [14] y [15]).

La convergencia de este nuevo esquema, depende del tipo de función $\gamma(x)$ que se seleccione, pues a pesar de que $D_{x}^{0}$ sea una representación finita de $d / d x$ en el espacio de funciones de la forma $(2.2)$, sus potencias $\left(D_{x}^{0}\right)^{k}$ no siempre son representaciones de las derivadas superiores. Un caso muy importante donde este hecho no representa mayor problema es cuando

$$
\gamma(x)=e^{-x^{2} / 2} .
$$

Con esta función, la solución de (2.10) consiste en el conjunto de los ceros del $N$-ésimo polinomio de Hermite $H_{N}(x)$ (ver por ejemplo [49]). Esta selección es útil en los problemas en donde la variable espacial puede tomar valores en $(-\infty, \infty)$ ya que el sistema ortogonal de funciones

$$
u_{n}(x)=e^{-x^{2} / 2} H_{n}(x)=\gamma(x) H_{n}(x), \quad n=0,1,2, \cdots,
$$

es una base para el espacio de funciones de cuadrado integrable, en la que una función de onda se puede expander como

$$
\Psi(x)=\sum_{n=0}^{\infty} c_{n} u_{n}(x)
$$

y las condiciones de frontera usuales $\Psi(-\infty)=\Psi(\infty)=0$ pueden satisfacerse.

La substitución de $d / d x$ por $D_{x}^{0}$ (construída con los ceros de Hermite) en (2.1) produce una ecuación matricial $N \times N$ cuya solución converge a la función de onda exacta evaluada en los nodos en la medida que los coeficientes $c_{n}$ de (2.19) convergen a cero para $n$ grande. En este sentido, la matriz $D_{x}^{0}$ (salvo la transformación de similitud dada en (2.14) resulta ser una proyección del operador $d / d x$ en el subespacio de funciones generado por $u_{n}(x)$, $n<N$ al que denotaremos por $U_{N-1}$, es decir

$$
U_{N-1}=\left\{\sum_{l<N} c_{l} u_{l}(x), c_{l} \in R\right\}
$$

Ya que $D_{x}^{0}$ es antisimétrica (como $d / d x$ ) y en general más simple que $\tilde{D}_{x}^{0}$, es conveniente usar esta representación cada vez que tengamos un problema definido en $(-\infty, \infty)$. 
A pesar de que las potencias de $D_{x}^{0}$ no siempre representan derivadas superiores, esta derivada discreta puede usarse en la ecuación de Schrödinger (2.1) sin mayor problema, pues solamente tendremos que aumentar el número de nodos en una unidad para obtener el mismo orden de convergencia, como se puede ver del siguiente razonamiento. Considérese una función de la forma $u(x)=\exp \left(-x^{2} / 2\right) f(x)$. Entonces $u^{\prime}(x)=e^{-x^{2} / 2}\left(f^{\prime}(x)-x f(x)\right)$, de manera si $f(x)$ es un polinomio de grado $m$, entonces la derivada de $u(x)$ es una función de la misma forma, pero ahora el polinomio que multiplica a la exponencial es de grado $m+1$; la segunda derivada de $u(x)$ será de la misma forma, pero con un polinomio de grado $m+2$; en general, la $k$-ésima derivada será de la misma forma pero con un polinomio de grado $m+k$. Por lo tanto, para que las potencias de la matriz $D_{x}^{0}$ [salvo la transformación de similitud dada en (2.14)], representen en forma exacta a las correspondientes derivadas evaluadas en los nodos, deberemos considerar subespacios de funciones más pequeños ( $\mathrm{y}$ perder rapidez en la convergencia) en la medida en que aumentemos el orden de derivación. En caso de tener una ecuación en primeras derivadas como la de Dirac, el uso de $D_{x}^{0}$ (o mejor dicho, de su generalización a más dimensiones obtenida más adelante) está libre de esta restricción.

La matriz $D_{x}^{0}$ tiene otras propiedades que la hacen adecuada para discretizar la derivada en las teorías cuánticas. Sus eigenvectores forman una matriz unitaria y simétrica $F_{x}$ cuyas entradas están dadas por

$$
\left(F_{x}\right)_{j k}=\sum_{l=0}^{N-1}(i)^{l} \varphi_{l}\left(p_{j}\right) \varphi_{l}\left(x_{k}\right)
$$

donde

$$
\varphi_{l}(x)=\sqrt{\frac{(N-1) ! 2^{N-1-l}}{N l !}} \frac{H_{l}(x)}{H_{N-1}(x)} .
$$

Aquí, $p_{j}$ es también una raíz de $H_{N}(x)$ pero representa un eigenvalor del impulso discreto [14]. Esta matriz resulta ser también una transformada discreta de Fourier, que puede ser generalizada para considerar transformadas discretas de tipo Fourier-Bessel (ver [17] y [18]). Sus propiedades básicas son

$$
\begin{aligned}
F_{x}^{-1} & =F_{x}^{\dagger} \\
-i D_{x}^{0} F_{x} & =F_{x} P^{x} \\
\lim _{N \rightarrow \infty}\left(F_{x}\right)_{j k} & =(-1)^{j+k} C_{N} e^{i p_{j} x_{k}},
\end{aligned}
$$

donde $P^{x}$ es una matriz diagonal con entradas $p_{j}$ a lo largo de la diagonal principal y

$$
C_{N}=2^{N-3 / 2}(\Gamma[(N+1) / 2])^{2} / N !
$$

Nótese que (2.23) es una discretización de la ecuación de eigenvalores del momemtum

$$
-i \frac{d}{d x} \phi_{p}(x)=p \phi_{p}(x)
$$


y por lo tanto corresponde a la discretización de la bien conocida regla cuántica

$$
p^{x} \rightarrow-i \frac{d}{d x},
$$

dando la representación finita del impulso

$$
P^{x}=-i D_{x}^{0}
$$

y asegurando el hecho de que $D_{x}^{0}$ es una representación finita de la derivada para la mecánica cuántica. Para ser rigurosos, debemos tomar a $\tilde{D}_{x}^{0}=S_{x} D_{x}^{0} S_{x}^{-1}$ [cf. (2.14)] como la discretización de la derivada, lo cual nos lleva a la representación del impulso

$$
P^{x}=-i S_{x} D_{x}^{0} S_{x}^{-1}
$$

y a que en las formas asintóticas de nuestros resultados (cuando $N \rightarrow \infty$ ), no aparezca un factor $(-1)^{j+k}$ que proviene de la matriz $F_{x}$ [ver por ejemplo (2.24)], pero este factor puede ser ignorado como si los resultados fueran obtenidos usando la matriz transformada $\tilde{D}_{x}^{0}$.

Es importante notar que la substitución $d / d x \rightarrow \tilde{D}_{x}^{0}$ es válida para funciones de onda definidas en todo $R$ (y que decaigan al infinito), lo que, como ya hemos visto, conduce a escoger los ceros de polinomios de Hermite como nodos para construir la representación matricial de la derivada. Por lo tanto, esta substitución no puede usarse si la ecuación de onda a discretizar tiene derivadas angulares (por ejemplo la ecuación de Schrödinger escrita en coordenadas esféricas para potenciales radiales), lo cual restringe excesivamente el uso de este método. El problema está en que los nodos de Hermite se expanden en la recta en la medida en que $N \rightarrow \infty$ [cubriendo los valores de la función de onda en $(-\infty, \infty)]$, y aunque en principio podríamos hacer una transformación lineal del intervalo que los contiene a $(-\pi, \pi)$, es mejor buscar una representación de la derivada angular $d / d \theta$ para funciones periódicas que preserve algunas relaciones cuánticas (como lo hace $D_{x}^{0}$ ) y que se construya con nodos totalmente contenidos en intervalos finitos como $(-\pi, \pi)$. Esto lo haremos en el siguiente capítulo y para terminar esta sección examinaremos la forma que adopta la relación de conmutación $\left[x, p^{x}\right]$ en nuestro espacio de proyección. Como ya lo hemos indicado, la proyección del operador $p^{x}$ resulta ser la matriz $-i \tilde{D}_{x}^{0}$ dada en (2.29), mientras que la proyección de $x$ es la matriz diagonal $X$ cuya diagonal principal contiene los puntos $x_{j}$. No es difícil ver que el conmutador de estas matrices es

$$
\left[X, P^{x}\right]=i\left(I_{N}-S_{x} O S_{x}^{-1}\right)
$$

donde $I_{N}$ es la matriz identidad de dimensión $N$ y $O$ es la matriz de proyección cuyas componentes son todas iguales a 1 (esta matriz proyecta todo vector $N$-dimensional a un vector con todas las componentes iguales). Para conocer la proyección de $U=S_{x} O S_{x}^{-1}$, recordemos la definición de $S_{x}$ [dada en (2.15)] y apliquemos $U$ al vector $u$ formado por una función de la forma $e^{-x^{2} / 2} f(x)$ [cf. (2.18)] donde $f(x)$ es un polinomio de grado a lo 
más $N-2$ evaluada en los nodos $x_{j}$. Ya que el coeficiente de $x^{N-1}$ de la representación lagrangiana de $f(x)$ debe anularse, cualquier $f(x)$ satisface

$$
\sum_{l=1}^{N} \frac{f\left(x_{l}\right)}{p^{\prime}\left(x_{l}\right)}=0
$$

donde $p(x)=\prod_{k=1}^{N}\left(x-x_{k}\right)[$ cf. $(2.7)]$, lo que puede escribirse como

$$
\left(O Q^{-1} f\right)_{j}=0, \quad j=1,2, \cdots, N .
$$

Por lo tanto, la aplicación de $U$ a $u$ da

$$
U f=\left(G Q O G^{-1} Q^{-1}\right) G f=(G Q) O Q^{-1} f=0,
$$

mostrando que $U$ proyecta los vectores de $U_{N-1}$ sobre la componente de grado más alto, es decir, sobre la componente correspondiente a $e^{-x^{2} / 2} x^{N-1}$. Esto muestra que la relación de conmutación (2.30) reproduce el resultado canónico siempre que la apliquemos en el subespacio $U_{N-2}$, lo que implica que, cuando $N \rightarrow \infty$, recobramos la relación continua

$$
\left[x, p^{x}\right]=i I
$$

donde $I$ es el operador identidad (en nuestro caso la matriz identidad). Este hecho también puede verse si calculamos el conmutador de $X$ y $P^{x}$ en otra base (ver [13]). Allí se obtiene que

$$
[X, P] \rightarrow i\left(\begin{array}{ccccc}
1 & 0 & 0 & \cdots & 0 \\
0 & 1 & 0 & \cdots & 0 \\
0 & 0 & 1 & \cdots & 0 \\
\vdots & \vdots & \vdots & \ddots & \vdots \\
0 & 0 & 0 & \cdots & 1-N
\end{array}\right)
$$

lo que muestra claramente la convergencia de nuestro resultado a la relación de conmutación canónica, ya que al crecer $N$, crece también el tamaño de la matriz, dejando el término que hace diferir a esta matriz de la identidad en la última componente.

\subsection{Discretización en varias variables}

Ya que el interés de este trabajo está en el estudio de un modelo discreto para el espaciotiempo en la Mecánica Cuántica relativista, deberemos extender los resultados de la sección anterior al caso de las $3+1$ variables del espacio-tiempo de Minkowski. En este caso, el espacio básico que sirve para construír estados cuánticos, denotado por $U\left(R^{4}\right)$, está dado por el conjunto de todas las funciones derivables en $R^{4}$ que caen más rápidamente que cualquier potencia de $r^{-1 / 2}=\left(x^{2}+y^{2}+z^{2}+t^{2}\right)^{-1 / 2}$ para $r \rightarrow \infty(c$, la velocidad de la luz está tomándose igual a 1) (ver por ejemplo [4], [5] y [32]). Una base de este espacio es

$$
\left\{e^{-\left(x^{2}+y^{2}+z^{2}+t^{2}\right) / 2} H_{n}(x) H_{m}(y) H_{l}(z) H_{k}(t),-\infty<x, y, z, t<\infty\right\}
$$


donde las $H$ 's son polinomios de Hermite de grados $n, m, l, k=0,1,2, \cdots$. Nótese que esta base está dada en términos de los productos tensoriales de las bases de los correspondientes espacios básicos univariados. Este hecho permite encontrar las representaciones finitas de las derivadas parciales como productos tensoriales matriciales de las proyecciones individuales en cada una de las coordenadas, generando así matrices de dimensión finita para las discretizaciones de cada una de las derivadas parciales.

Por el momento, para obtener una generalización del modelo presentado en la primera sección e ilustrar la técnica de trabajo, sólo consideraremos dos variables no angulares, que en forma genérica denotaremos $x$ y $y$.

La proyección en $x$ se hará en sobre $U_{N-1}\left[\right.$ cf. (2.20)], y la de $y$ sobre $U_{M-1}$, con $N$ y $M$ enteros, por lo que $U\left(R^{2}\right)$ estará proyectado sobre $U_{N-1} \otimes U_{M-1}=U_{(N-1)(M-1)}$. Las nuevas representaciones podrán obtenerse como productos tensoriales de las representaciones individuales en cada subespacio (en el Apéndice A se pueden ver algunas propiedades que usaremos de los productos tensoriales matriciales). Para conocer las formas que adoptan ahora los operadores $x$ y $y$, debemos recordar que la forma de los elementos de toda representación se obtienen con la aplicación del operador sobre los elementos de la base en que se esté representando. Sea $\left|e_{j}\right\rangle$ un elemento de la nueva base formada con productos de las bases ortonormales $\mid a_{l}>$ y $\mid b_{m}>$ de acuerdo a

$$
\left|e_{j}\right\rangle=\left|a_{l} b_{m}\right\rangle=\left|a_{l}\right\rangle\left|b_{m}\right\rangle,
$$

donde el índice $j$ está dado en función de $l$ y $m$ en forma apropiada y sea $\mathbf{A}$ un operador aplicando sobre uno de los subespacios, digamos el de base $\left|a_{l}\right\rangle$. El elemento $i j$ de la representación es

$$
\left.\left.\left.<b_{n} a_{k}|\mathbf{A}| a_{l} b_{m}\right\rangle=<b_{n}\left(<a_{k}|\mathbf{A}| a_{l}\right\rangle\right)\left|b_{m}\right\rangle=<b_{n} \mid b_{m}>\left(<a_{k}|\mathbf{A}| a_{l}\right\rangle\right) .
$$

Teniendo en cuenta la ortonormalidad de $\mid b_{m}>$ y dependiendo de la forma en que se haya ordenado la base (2.37) (es decir, de la relación existente entre los índices $j, l$ y $m$ y de cuál índice sea el que corra más rápidamente), la representación de $\mathbf{A}$ en el nuevo espacio tendrá alguna de las formas tensoriales siguientes

$$
I \otimes A \quad 0 \quad A \otimes I,
$$

donde $I$ representa la matriz identidad y $A$ es la representación de $\mathbf{A}$ en la base $\left|a_{l}\right\rangle$. En nuestro modelo, seleccionaremos a la base de $x$ como la que corra más rápidamente (en el caso de $3+1$ variables tomaremos el orden siguiente: primero $x$, después $y$ y $z, \mathrm{y}$ finalmente $t$ ), así, de acuerdo a lo anterior, el operador $x$ tendrá la nueva representación $\mathbf{X}$ dada por

$$
\mathbf{X}=I_{M} \otimes X_{N}
$$

mientras que $y$ adoptará la forma

$$
\mathbf{Y}=Y_{M} \otimes I_{N}
$$

donde las matrices $I_{M}$ y $I_{N}$ son las matrices identidad de dimensión $M$ y $N$ respectivamente dando lugar a que $\mathbf{X}$ y $\mathbf{Y}$ sean matrices $N M \times N M$. Nótese que $\mathbf{X}$ y $\mathrm{Y}$ conmutan, 
al igual que los operadores $x$ y $y$, por lo que pueden ser diagonalizados simultáneamente. Las entradas diagonales de $X_{N}$ y $Y_{M}$ son sus eigenvalores $\left\{x_{1}, x_{2}, \cdots, x_{N}\right\}$ con degeneración $M$ cada uno de ellos y $\left\{y_{1}, y_{2}, \cdots, y_{M}\right\}$ con degeneración $N$ cada uno de estos últimos. Los puntos $x_{j}$ y $y_{j}$ corresponden a ceros de $H_{N}(x)$ y $H_{M}(y)$ respectivamente. Los subíndices de las matrices en (2.40) y (2.41) se refieren explícitamente a la dimensión de cada una de ellas, pero con el fin de no complicar la notación, no los escribiremos sino cuando se requiera distinguir entre las dimensiones.

De la misma forma, las componentes del impulso $p^{x}$ y $p^{y}$ tendrán las representaciones

$$
\mathbf{p}^{x}=I_{M} \otimes P_{N}^{x} \quad \mathbf{P}^{y}=P_{M}^{y} \otimes I_{N}
$$

[cf. (2.28)] que también conmutan y por lo tanto son diagonalizables simultáneamente. En este caso sus eigenvalores son (sin considerar orden alguno) $\left\{p_{1}^{x}, p_{2}^{x}, \cdots, p_{N}^{x}\right\}$, con degeneración $M$, y $\left\{p_{1}^{y}, p_{2}^{y}, \cdots, p_{M}^{y}\right\}$, con degeneración $N$, respectivamente. Como se vió en la primera sección, cada uno de estos valores propios son también ceros de polinomios de Hermite (salvo una constante dimensional de impulso para los valores $p_{j}^{x}, p_{j}^{y}$, y de posición para los eigenvalores de $\mathbf{X}$ y $\mathbf{Y}$ ).

Así, no es difícil ver que las matrices que representan a las derivadas $\partial / \partial x, \partial / \partial y$, son

$$
\mathbf{D}_{x}=I_{M} \otimes D_{x}^{0} \quad \mathbf{D}_{y}=D_{y}^{0} \otimes I_{N}
$$

respectivamente, salvo una transformación de similitud [cf. (2.14)] dada por

$$
\mathbf{S}=S_{y} \otimes S_{x}
$$

donde $S_{y}=G_{y} Q_{y}$ y $S_{x}=G_{x} Q_{x}$ (los subíndices hacen referencia a la variable en que deben ser calculadas las matrices $G$ y $Q$ ). Por simplicidad podemos no tomar en cuenta esta transformación en nuestras ecuaciones discretas a cambio de que en las expresiones asintóticas que obtengamos ignoremos el factor $(-1)^{j+k}$ proveniente de cada una de las matrices $S_{x}$ y $S_{y}$. Las matrices $D_{x}$ y $D_{y}$ de (2.43) son de dimensión $N$ y $M$ respectivamente y ambas tienen la estructura de (2.6) sólo que la primera se construye con el conjunto de nodos $\left\{x_{i}, x_{2}, \cdots, x_{N}\right\}$ y la segunda con $\left\{y_{i}, y_{2}, \cdots, y_{M}\right\}$. Por propiedades del producto de Kronecker, las matrices $\mathbf{D}_{x}$ y $\mathbf{D}_{y}$ conmutan, lo cual era de esperarse (ver el Apéndice A).

Para asegurar el hecho de que $\mathbf{D}_{x}$ y $\mathbf{D}_{y}$ son representaciones de las derivadas parciales, y que pueden usarse junto con $\mathbf{X}$ y $\mathbf{Y}$, para resolver problemas diferenciales parciales en forma numérica, puede consultase el Apéndice $\mathrm{B}$ donde se da una derivación más rigurosa de este esquema multidimensional.

Procedamos ahora a obtener la representación discreta de la transformación unitaria entre la base continua del vector de posición $\vec{r}=(x, y)$ y la del impulso $\vec{p}=\left(p^{x}, p^{y}\right)$, es decir, la matriz que representa en $U\left(R^{2}\right)$ la transformada de Fourier que lleva cualquier función de las variables $x$ y $y$ a la correspondiente función de $p^{x}$ y $p^{y}$. Ya que el núcleo de la transformada está compuesto por el producto de los núcleos unidimensionales respectivos

$$
e^{-i \vec{r} \cdot \vec{p}}=e^{i x p^{x}} e^{i y p^{y}}
$$


por lo que, de acuerdo a la discusión del principio de esta sección, su matriz representativa será el producto tensorial

$$
\mathbf{F}=F^{y} \otimes F^{x}
$$

donde las matrices $F^{x}$ y $F^{y}$ son las representativas de las transformadas de Fourier en cada subsespacio y ambas tienen la estructura (2.21). Usando propiedades del producto de Kroenecker (ver Apéndice A), puede verse que esta matriz es unitaria. Además, no es difícil ver que todas las relaciones del álgebra para una variable, se heredan a este caso. Considérese por ejemplo el producto $-i \mathbf{D}_{x} \mathbf{F}$ que es la representación discreta (asintóticamente) de $-i \partial\left(e^{i \vec{r} \cdot \vec{p}}\right) / \partial x$. Así, tendremos que

$$
-i \mathbf{D}_{x} \mathbf{F}=\mathbf{F P} \mathbf{P}^{x} .
$$

La ecuación anterior corresponde una generalización de (2.23). De aquí podemos concluir nuevamente que la matriz diagonal $\mathrm{P}^{x}$ es unitariamente similar a $-i \mathbf{D}_{x}$. En la misma forma se considera la coordenada $y$ para producir resultados análogos. Ahora consideraremos dos propiedades de la matriz $\mathbf{F}$ importantes para el desarrollo de este trabajo. La primera concierne con la forma asintótica de sus elementos cuando $N, M \rightarrow \infty$. Como puede verse en el Apéndice $\mathrm{A}$, el elemento $q s$ del producto directo de la matriz $F^{y}$ y la matriz $F^{x}$ puede escribirse en términos de los elementos individuales de estas matrices por medio de

$$
\left(F^{y} \otimes F^{x}\right)_{q s}=F_{n k}^{y} F_{l j}^{x}
$$

donde los índices $q, s, n, k, l$ y $j$ están ordenados de acuerdo a

$$
q=l+(n-1) N, \quad s=j+(k-1) N,
$$

con $l, j=1,2, \cdots, N$, y $n, k=1,2, \cdots, M$.

Por lo tanto, esta ecuación, (2.46) y (2.24) producen la forma asintótica puntual

$$
\lim _{N, M \rightarrow \infty} \mathbf{F}_{q s}=C_{N} C_{M} e^{i p_{n}^{y} y_{k}} e^{i p_{l}^{x} x_{j}}
$$

en donde hemos suprimido el factor alternante en signo que aparece en $(2.24 \mathrm{y}$ que se debe a la falta de la matriz de similitud (2.44) en esa ecuación.

La segunda propiedad de $\mathbf{F}$ tiene que ver con su aplicación como una transformada discreta de Fourier en dos variables, que es una generalización de la transformada discreta en una variable y para la cual se ha estimado el término de error (ver [17] y [18]).

Una vez teniendo a la mano el esquema de discretización en dos variables, la extensión a un número mayor es directa aunque engorrosa, ya que el uso de los subíndices dificulta el manejo de esta técnica. Por tal motivo trataremos de usar más la forma matricial de las ecuaciones que las formas en que se exhibe explícitamente la dependencia entre elementos de matrices.

Nuestras observables serán ahora $x^{1}, x^{2}, \cdots, x^{q}$, y los respectivos impulsos $p^{1}, p^{2}, \cdots, p^{q}$. La proyección de $x^{1}$ se hará sobre $U_{N_{1}-1}$ y la de $x^{2}$ sobre $U_{N_{2}-1}$, y así sucesivamente. El orden para construír el álgebra, será el dado por los índices de las variables: primero $x^{1}$, 
después $x^{2}$, etc. De esta manera si $X^{1}, X^{2}, \cdots, X^{q}$ son las representaciones discretas de las variables en cada uno de sus subespacios individuales, tendremos que la representación de $x^{1}$ en $U_{\tilde{N}}$, donde $\tilde{N}=\left(N_{1}-1\right)\left(N_{2}-1\right) \cdots\left(N_{q}-1\right)$, está dada por

$$
\mathbf{X}^{1}=I_{N_{q}} \otimes \cdots \otimes I_{N_{2}} \otimes X^{1}
$$

ecuación análoga a (2.40). Nuevamente los subíndices de las matrices indican sus dimensiones. Las demás representaciones están dadas en forma similar:

$$
\begin{gathered}
\mathbf{X}^{2}=I_{N_{q}} \otimes \cdots \otimes X^{2} \otimes I_{N_{1}}, \\
\vdots \\
\mathbf{X}^{q}=X^{q} \otimes \cdots \otimes I_{N_{2}} \otimes I_{N_{1}} .
\end{gathered}
$$

Esta misma estructura tendrán las representaciones de los impulsos

$$
\mathbf{P}^{k}=I_{N_{q}} \otimes \cdots I_{N_{k+1}} \otimes P^{k} \otimes I_{N_{k-1}} \otimes \cdots I_{N_{1}}
$$

donde $P^{k}$ es la matriz diagonal $N_{k} \times N_{k}$, proyección individual de la observable $p^{k}$, con eigenvalores $p_{1}^{k}, p_{2}^{k}, \cdots, p_{N_{k}}^{k}$; el índice $k$ corre sobre el número de variables. Por supuesto, las derivadas parciales también tendrán una representación similar:

$$
\mathbf{D}_{k}=I_{N_{q}} \otimes \cdots I_{N_{k+1}} \otimes D_{k}^{0} \otimes I_{N_{k-1}} \otimes \cdots I_{N_{1}}
$$

De la misma forma, es ahora una transformada de Fourier multidimensional la que lleva la base de la posición a la de impulsos. Su núcleo está dado por

$$
e^{i \vec{r} \cdot \vec{p}}=\prod_{k=1}^{q} e^{i x^{k} p^{k}}
$$

por lo que, su matriz representativa será el producto

$$
\mathrm{F}=F^{q} \otimes \cdots \otimes F^{2} \otimes F^{1}
$$

en donde, de forma análoga al caso bidimensional, la matriz genérica $F^{k}$ es una matriz de $N_{k} \times N_{k}$ y tiene la estructura (2.21), construyéndose con las raíces de Hermite $\left\{x_{1}^{k}, x_{2}^{k}, \cdots, x_{N_{k}}^{k}\right\}$, eigenvalores de la observable discreta $X^{k}$. Esta matriz es unitaria y también satisface una relación asintótica similar a (2.50). Las matrices $\mathbf{D}_{k}$, que representan derivadas parciales también cumplen relaciones como (2.47):

$$
-i \mathbf{D}_{k} \mathbf{F}=\mathbf{F P}^{k}, \quad k=1,2 \cdots, q
$$

Esto significa que dichas matrices son diagonalizadas simultánemente por la matriz $\mathbf{F}$, y son realizaciones discretas de las ecuaciones de eigenvalores de los impulsos [cf. (2.28) y (2.47)]. Igual procedimiento se sigue para interpretar a $\mathbf{F}$ como una transformada discreta de Fourier multidimensional. 
Desde el punto de vista matemático, estos resultados nos permiten, primero, discretizar (o proyectar) ecuaciones diferenciales parciales (cuyas soluciones puedan escribirse como series de Fourier multivariadas) mediante las substituciones

$$
x^{k} \rightarrow \mathbf{X}^{k} \quad \frac{\partial}{\partial x^{k}} \rightarrow \mathbf{D}_{k}
$$

y segundo, al igual que en el caso continuo, resolverlas mediante una TDF multivariada. Desde el punto de vista de la Física discreta, tenemos un método que mantiene la representación en coordenadas del operador impulso en las variables discretas y que (como se verá más adelante) produce convergencia hacia los resultados continuos en problemas cuánticos clásicos o relativistas.

Es importante notar que las variables $x^{k}$ no necesariamente son de tipo cartesiano. Podemos suponer que $x^{k}$ representa una variable angular, siempre que el espacio de proyección y los nodos con que se construya la matriz representativa de la derivada se escojan de acuerdo a los resultados de la sección 3.1.

También $x^{k}$ puede representar al tiempo $t$. Acordemos denotar las coordenadas del espacio-tiempo de Minkowski en la forma: $x$ por $x^{1}, y$ por $x^{2}, z$ por $x^{3}$ y $t$ por $x^{4}$. Ya que (2.36) es una base para las funciones de onda en este espacio (donde la variable $t$ corre de $-\infty$ a $\infty$ y no se distingue en principio de una coordenada espacial), los nodos temporales también resultan ser ceros de Hermite, lo cual significa que aceptamos la existencia de un operador asociado al tiempo cuya representación en nuestro espacio de proyección tiene eigenvalores discretos que corresponden a estos puntos de la misma forma que las representaciones discretas de $x, y$ y $z$ también tienen como eigenvalores a los ceros de polinomios de Hermite. Sobre otras maneras de asignar eigenvalores temporales, véanse las referencias [27], [29], [39]. Sin embargo, para que nuestro esquema reproduzca el signo positivo de la representación de la energía como derivada temporal y el negativo en la de los impulsos como derivadas espaciales de acuerdo a las reglas cuánticas

$$
p^{k}=-i \frac{\partial}{\partial x^{k}} \quad E=i \frac{\partial}{\partial t},
$$

$k=1,2,3$, debemos redefinir la matriz $\mathbf{F}$ en la forma

$$
\mathbf{F}=\left(F^{4}\right)^{*} \otimes F^{3} \otimes F^{2} \otimes F^{1},
$$

en donde $*$ significa complejo conjugado, ya que de este modo tendremos

$$
-i \mathbf{D}_{k} \mathbf{F}=\mathbf{F P}^{k}, \quad i \mathbf{D}_{4} \mathbf{F}=\mathbf{F E},
$$

donde $k=1,2,3$, y $\mathbf{E}=\mathbf{P}^{4}$ es la matriz diagonal que lleva los eigenvalores de la energía en su diagonal principal y que corresponden a raíces de Hermite para el caso de una partícula libre. Anticipándonos un poco, debemos decir que en este esquema discreto las relaciones relativistas entre energía, impulso y masa también se mantienen en sus contrapartes discretas, como se puede ver en el Capítulo 5. 
Teniendo en cuenta que $\mathbf{F}$ es una matriz simétrica, mientras que $\mathbf{D}_{j}, j=1,2,3,4$, es antisimétrica, al transponer (2.61) se obtienen las ecuaciones

$$
\mathbf{F D}_{k}=-i \mathbf{P}^{k} \mathbf{F}, \quad \mathrm{FD}_{4}=i \mathbf{E F},
$$

que reflejan el hecho de que $\mathbf{F}$ es una transformada de Fourier discreta, pues actúa sobre las derivadas en la misma forma que la transformada de Fourier continua. 


\section{Capítulo 3}

\section{El impulso angular en un espacio discreto}

\subsection{Rotaciones discretas}

Para que nuestro método de discretización pueda ser aplicado a problemas físicos relevantes, es necesario contar con una manera de discretizar variables angulares. En esta sección encontraremos una representacón matricial finita para la derivada angular (la derivada de funciones periódicas univariadas) y mostraremos que esa matriz es una representación discreta de la componente $z$ del impulso angular y está relacionada con un generador de rotaciones discretas asociado con un subgrupo del grupo de rotaciones.

Consideremos un conjunto completo de estados cuánticos en un espacio de dimensión finita, $\left|\varphi_{j}\right\rangle$, por determinarse más tarde. Así, el operador $\Delta$ que produce una permutación cíclica (salvo por un factor de fase) del conjunto $\left|\varphi_{1}\right\rangle, \cdots,\left|\varphi_{N}\right\rangle$,

$$
\Delta\left|\varphi_{j}\right\rangle= \begin{cases}e^{i \gamma_{j+1}}\left|\varphi_{j+1}\right\rangle, & j \neq N, \\ e^{i \gamma_{1}}\left|\varphi_{1}\right\rangle, & j=N,\end{cases}
$$

es la matriz de elementos $\left\langle\varphi_{j}|\Delta| \varphi_{k}\right\rangle=\Delta_{j k}$ dados por

$$
\left(\Delta_{j k}\right)=\left(\begin{array}{cccccc}
0 & 0 & 0 & \cdots & 0 & e^{i \gamma_{1}} \\
e^{i \gamma_{2}} & 0 & 0 & \cdots & 0 & 0 \\
0 & e^{i \gamma_{3}} & 0 & \cdots & 0 & 0 \\
\vdots & \vdots & \vdots & \ddots & \vdots & \vdots \\
0 & 0 & 0 & \cdots & 0 & 0 \\
0 & 0 & 0 & \cdots & e^{i \gamma_{N}} & 0
\end{array}\right) .
$$

Por lo tanto,

$$
\Delta^{N}=e^{i \Gamma} I_{N}
$$

donde $\Gamma=\sum_{j=1}^{N} \gamma_{j}$, y $I_{N}$ es la matriz identidad de dimensión $N$. Ya que el determinante de $\Delta$ es

$$
\operatorname{det} \Delta=(-1)^{N+1} e^{i \Gamma},
$$


para que $\Delta$ represente una rotación propia y genere un subgrupo finito del grupo de rotaciones, deberemos tener que

$$
\Gamma= \begin{cases}2 l \pi, & N \text { impar } \\ (2 l+1) \pi, & N \text { par }\end{cases}
$$

con $l$ entero. Así, (3.3) toma la forma

$$
\Delta^{N}= \begin{cases}1, & N \text { impar } \\ -1, & N \text { par }\end{cases}
$$

mostrando que el conjunto de potencias de $\Delta$ es una representación del grupo cíclico de orden $N$ o $2 N$ para $N$ impar o par, respectivamente (en el caso par es una representación bivaluada del grupo finito de rotaciones). Por lo tanto, cada eigenvalor de cualquier potencia de $\Delta$ es una raíz racional de la unidad. Si fijamos las fases $\gamma_{j}$ adecuadamente, podemos escribir (3.2) en una forma más manejable, pero debido a (3.6) es necesario considerar el caso par e impar por separado. Por lo tanto podemos hacer

$$
\Delta_{j k}=\left\{\begin{array}{lll}
\delta_{j N}, & \text { para } & j=1, \\
\delta_{j, k+1}, & \text { para } & j \geq 2,
\end{array}\right.
$$

para $N$ impar, y

$$
\Delta_{j k}= \begin{cases}-\delta_{j N}, & \text { for } j=1, \\ \delta_{j, k+1}, & \text { for } j \geq 2 .\end{cases}
$$

para $N$ par. No es difícil ver que el polinomio característico de $\Delta$ en la variable $\lambda$ es $(-\lambda)^{N}+1=0$ para toda $N$, y que el vector de componentes $e^{-i k a_{j}} / \sqrt{N}, k=1,2, \cdots, N$, es el $j$-́simo eigenvector normalizado de $\Delta$ con eigenvalor $\lambda_{j}=e^{i a_{j}}$, donde

$$
a_{j}=\frac{2 \pi j}{N}, \quad j \in Z_{N}
$$

$\mathrm{y}$

$$
Z_{N}= \begin{cases}0, \pm 1, \pm 2, \cdots, \pm n, & N=2 n+1 \\ \pm 1 / 2, \pm 3 / 2, \cdots, \pm(2 n-1) / 2, & N=2 n\end{cases}
$$

Ya que $\Delta$ es unitaria, define una matriz hermitiana $A$ a través de

$$
\Delta=e^{i A}
$$

cuyos eigenvalores están dados por (3.9) (nótese que la traza de $A$ se anula) y sus eigenvectores, denotados por $\left|a_{j}\right\rangle$, son los mismos que aquellos de $\Delta$. Por lo tanto, la matriz que diagonaliza simultáneamente $\Delta$ y $A$ es

$$
\left\langle\varphi_{k} \mid a_{j}\right\rangle=\frac{1}{\sqrt{N}} e^{-i k a_{j}}, \quad k, j=1,2, \cdots, N
$$


En el caso continuo $A$ es proporcional al ángulo de rotación por la componente del impulso angular $L_{z}$. Para encontrar el caso análogo discreto, requerimos calcular los elementos de $A$ en la base de $\varphi$. Esto puede hacerse usando el hecho que

$$
A_{j k}=\left\langle\varphi_{j}|A| \varphi_{k}\right\rangle=\sum_{l=1}^{N}\left\langle\varphi_{j} \mid a_{l}\right\rangle a_{l}\left\langle a_{l} \mid \varphi_{k}\right\rangle=\frac{2 \pi}{N^{2}} \sum_{l \in I_{N}} l e^{-i(j-k) a_{l}} .
$$

Nótese que los elementos diagonales se anulan. Para $N=2 n+1$ tenemos

$$
A_{j k}=-\frac{4 \pi}{N^{2}} i \sum_{l=1}^{n} l \sin \frac{2 \pi l(j-k)}{N},
$$

mientras que para $N=2 n$,

$$
A_{j k}=-\frac{4 \pi}{N^{2}} i \sum_{l=1}^{n}(l-1 / 2) \sin \frac{2 \pi(l-1 / 2)(j-k)}{N} .
$$

Estas sumas pueden calcularse fácilmente $[30]$ y, para $N$ par o impar, son iguales a

$$
A_{j k}=\left\langle\varphi_{j}|A| \varphi_{k}\right\rangle= \begin{cases}0, & j=k, \\ \frac{i(-1)^{j+k} \pi / N}{\sin \frac{\pi(j-k)}{N}}, & j \neq k\end{cases}
$$

Ahora podemos establecer una relación entre $A$ y la representación matricial $N$-dimensional de la derivada de polinomios trigonométricos buscada. Escojamos primero el caso impar $N=2 n+1$.

\subsubsection{Caso impar}

Sea $\tau_{n-1}(x)$ el espacio de polinomios trigonométricos de grado menor que $n$ en la variable angular $x$ (por simplicidad cambiemos la notación de la variable angular en lo que resta de esta sección). Es bien conocido (ver por ejemplo [50]) que cualquier polinomio trigonométrico $f \in \tau_{n-1}(x)$ puede ser determinado de manera única por sus valores en $2 n+1$ puntos arbitrarios $-\pi<x_{1}<x_{2}<\cdots<x_{2 n+1} \leq \pi$, mediante la fórmula

$$
f(x)=\sum_{k=1}^{2 n+1} f\left(x_{k}\right) \frac{t_{k}(x)}{t_{k}\left(x_{k}\right)}
$$

donde los polinomios $t_{k} \in \tau_{n-1}(x)$ están dados por

$$
t_{k}(x)=\prod_{l \neq k}^{2 n+1} \sin \frac{\left(x-x_{l}\right)}{2} .
$$


Esta expresión puede escribirse de otra forma si conocemos los valores de $d f(x) / d x$ en los nodos. La diferenciación de (3.18) y manipulación algebraica del resultado produce

$$
t_{k}^{\prime}\left(x_{j}\right)=\left\{\begin{array}{ll}
t^{\prime}\left(x_{k}\right) \sum_{l=1}^{2 n+1} \cot \frac{\left(x_{k}-x_{l}\right)}{2}, & j=k, \\
t^{\prime}\left(x_{j}\right) \csc \frac{\left(x_{j}-x_{k}\right)}{2}, & j \neq k,
\end{array},\right.
$$

donde

$$
t(x)=t_{k}(x) \sin \frac{\left(x-x_{k}\right)}{2}=\prod_{l=1}^{2 n+1} \sin \frac{\left(x-x_{l}\right)}{2} .
$$

Por lo tanto, $f^{\prime}\left(x_{j}\right)$ toma la forma

$$
f^{\prime}\left(x_{j}\right)=\frac{1}{2} t^{\prime}\left(x_{j}\right) \sum_{k=1}^{2 n+1} \frac{f\left(x_{k}\right)}{\sin \frac{\left(x_{j}-x_{k}\right)}{2} t^{\prime}\left(x_{k}\right)}+\frac{1}{2} f\left(x_{j}\right) \sum_{k=1}^{2 n+1} \cot \frac{\left(x_{j}-x_{k}\right)}{2} .
$$

Ya que $f^{\prime}(x)$ es nuevamente un elemento de $\tau_{n-1}(x)$, esta ecuación se escribe matricialmente en la forma (2.13)

$$
f^{\prime}=\tilde{D}_{\varphi} f \quad \tilde{D}_{\varphi}=T D_{\varphi} T^{-1},
$$

donde ahora

$$
\left(D_{\varphi}\right)_{i j}=\left\{\begin{array}{ll}
\sum_{l=1}^{N} \frac{1}{2} \cot \frac{\left(x_{i}-x_{l}\right)}{2}, & i=j, \\
\frac{1}{2} \csc \frac{\left(x_{i}-x_{j}\right)}{2}, & i \neq j,
\end{array} \quad T_{i j}=t^{\prime}\left(x_{i}\right) \delta_{i j}, \quad i, j=1, N .\right.
$$

Si escogemos los nodos de tal forma que

$$
x_{j}=-\pi+\frac{2 \pi j}{N}, \quad j=1,2, \cdots, N
$$

puede mostrarse que, independientemente de la paridad de $N$, el producto (3.18) y las sumas $\left(D_{\varphi}\right)_{j j}$, evaluadas en los nodos (3.24), satisfacen las ecuaciones

$$
\frac{t_{j}\left(x_{j}\right)}{t_{k}\left(x_{k}\right)}=\frac{t^{\prime}\left(x_{j}\right)}{t^{\prime}\left(x_{k}\right)}=(-1)^{j+k}
$$

$\mathrm{y}$

$$
\left(D_{\varphi}\right)_{j j}=\sum_{k=1}^{N} \cot \frac{(j-k) \pi}{N}=0, \quad j=1,2, \cdots, N .
$$

Por lo tanto, substituyendo estas expresiones en (3.21) y tomando en cuenta (3.16), obtenemos que con esta selección de nodos y $N=2 n+1$,

$$
A=i \frac{2 \pi}{N} \tilde{D}_{\varphi}^{0}=-\frac{2 \pi}{N} L_{z}
$$


donde hemos definido a $\tilde{D}_{\varphi}^{0}$ como la matriz $\tilde{D}_{\varphi}$ evaluada en los nodos $(3.24)$ y a $L_{z}$ como

$$
L_{z}=-i \tilde{D_{\varphi}^{0}}
$$

Por lo tanto, $\Delta$, el operador de rotación discreto toma la forma

$$
\Delta=e^{-\frac{2 \pi}{N} D_{\varphi}^{0}}=e^{-i \epsilon L_{z}}
$$

con $\epsilon=2 \pi / N$. De esta manera vemos que al igual que en el caso continuo, el argumento de la exponencial resulta ser proporcional al ángulo de rotación (en este caso $\epsilon$ ) por la derivada angular. Debido a (3.27), (3.9) y (3.12), $L_{z}$ tiene los $2 n+1$ eigenvalores

$$
\{-n,-n+1, \cdots,-1,0,1, \cdots, n-1, n\},
$$

y los correspondientes eigenvectores normalizados $|m\rangle, m=-n, \cdots, n$ cuyas componentes (en la base $\varphi$ ) son

$$
\left\langle\varphi_{k} \mid m\right\rangle=\frac{1}{\sqrt{N}} e^{-i m \varphi_{k}}
$$

donde $\varphi_{k}=k \epsilon, k=1,2, \cdots, N$. Así, el hecho de que el operador impulso pueda definirse en este espacio de dimensión finita a través de (3.28) y (3.29) es una consecuencia de que $\tilde{D}_{\varphi}^{0}$ es una proyección de $d / d \varphi$ sobre dicho espacio.

\subsubsection{Caso par}

Esencialmente, este caso difiere del anterior unicamente en el espacio de proyección usado. Principiemos considerando una función de la forma

$$
g(x)=\sin (x / 2) f(x),
$$

donde $f \in \tau_{n-1}(x)$. Sean $-\pi<x_{1}^{*}<x_{2}^{*}<\cdots<x_{2 n-1}^{*} \leq \pi N-1$ puntos arbitrarios, differentes de cero. Interpolando $f$ en estos puntos, se obtiene

$$
g(x)=\sum_{k=1}^{N-1} f\left(x_{k}^{*}\right) \frac{\sin (x / 2) t_{k}^{*}(x)}{t_{k}^{*}\left(x_{k}^{*}\right)}=\sum_{k=1}^{N-1}\left[\sin \left(x_{k}^{*} / 2\right) f\left(x_{k}^{*}\right)\right] \frac{\sin (x / 2) t_{k}^{*}(x)}{\sin \left(x_{k}^{*} / 2\right) t_{k}^{*}\left(x_{k}^{*}\right)}
$$

es decir,

$$
g(x)=\sum_{k=1}^{N-1} g\left(x_{k}^{*}\right) \frac{\sin (x / 2) t_{k}^{*}(x)}{\sin \left(x_{k}^{*} / 2\right) t_{k}^{*}\left(x_{k}^{*}\right)} .
$$

Las funciones $t_{k}^{*}(x)$ son polinomios Gaussianos dados por un producto como (3.18) con $N-1$ nodos $x_{j}^{*}$. Ahora consideremos el conjunto de puntos formado por la unión todos los $x_{j}^{*}$ y cero y denotémoslos (ordenadamente) por $x_{j}, j=1,2, \cdots, 2 n$ y por $t_{k}(x)$ a los polinomios interpolatorios correspondientes. Entonces, tomando en cuenta que $g(0)=0$, (3.34) toma la forma

$$
g(x)=\sum_{k=1}^{2 n} g\left(x_{k}\right) \frac{t_{k}(x)}{t_{k}\left(x_{k}\right)}
$$


i.e., tenemos ahora una fórmula interpolatoria para funciones del tipo (3.32) en los nodos $x_{j}$, así que los razonamientos del caso impar se pueden aplicar a (3.35) para dar una expresión similar a (3.21) para $g(x)$. Por lo tanto, para los $2 n$ nodos formados con cualesquiera $2 n-1$ puntos differentes de $(-\pi, \pi]$ y cero, la matriz

$$
\tilde{D}_{\varphi}=T D_{\varphi} T^{-1}
$$

con las definiciones de (3.23) es una representación finita de la derivada para funciones de la forma (3.32). Más aún, tomando en cuenta que $\cos x / 2=\sin [(x+\pi) / 2]$, que $\pi$ puede ser tomado como uno de los nodos y que esta función se anula en $x=\pi$, podemos manipular la función $h(x)=\cos (x / 2) f(x)$ en la misma forma que (3.32) para concluir que la $k$-ésima potencia de (3.36) construída con $2 n$ puntos distintos del intervalo semiabierto $(-\pi, \pi]$ y cero, con $\pi$ un elemento de tal conjunto, es una proyección de la $k$-ésima derivada sobre el subespacio de funciones del tipo

$$
e^{i x / 2} f(x)
$$

donde $f \in \tau_{n-1}(x)$. Ahora, si pedimos que los nodos tengan la forma (3.24), 0 y $\pi$ forman parte de ellos y los razonamientos anteriores continúan siendo válidos y producen las mismas fórmulas (3.27)-(3.29) para las relaciones entre $A, \tilde{D_{\varphi}^{0}}, L_{z}$ y $\Delta$, sólo que ahora con $N=2 n$. Por lo tanto, ahora tenemos que $L_{z}$ tiene los $2 n$ eigenvalores

$$
\{-(2 n-1) / 2,-(2 n-3) / 2, \cdots,-1 / 2,1 / 2, \cdots,(2 n-3) / 2,(2 n-1) / 2\},
$$

y sus eigenvectores $|m\rangle, m=-(2 n-1) / 2, \cdots,(2 n-1) / 2$ están dados por

$$
\left\langle\varphi_{k} \mid m\right\rangle=\frac{1}{\sqrt{N}} e^{-i m \varphi_{k}}
$$

donde $\varphi_{k}=k \epsilon$.

Resumiendo los resultados de esta sección, hemos encontrado que un subgrupo finito del grupo de rotaciones tiene asociada una representación matricial finita de la derivada de funciones periódicas que a su vez está asociada, de acuerdo a los postulados cuánticos, a una componente del impulso angular proyectado en el subespacio generado por dichas funciones. Esto completa nuestra descripción discreta de las reglas cuánticas.

\subsection{Una matriz finita para $L^{2}$}

Con los resultados de las secciones 2.2 y 3.1, podemos encontrar una proyección del cuadrado del impulso angular $L^{2}$ de un sistema descrito por tres grados de libertad clásicos sobre un espacio lineal de dimensión finita y establecer su correspondiente problema de eigenvalores. También es posible por supuesto, encontrar las proyecciones de las componentes individuales del impulso angular y de sus relaciones de conmutación, pero este cálculo lo dejaremos para la siguiente sección. 
Ya que las eigenfunciones de $L^{2}$ (los armónicos esféricos $Y_{n}^{m}(\theta, \varphi)$ ) son polinomios trigonométricos en las variables $\theta$ y $\varphi$, y nuestro método de proyección produce resultados exactos para este tipo de polinomios, es de esperarse que el problema de eigenvalores en el espacio de proyección reproduzca los eigenvalores y eigenfunciones del caso continuo. De acuerdo a los resultados de 2.2 y 3.1 , requerimos de las matrices $\tilde{D}_{\theta}$ de dimensión $N$, y $\tilde{D}_{\varphi}$ de dimensión $M$ para proyectar a $d / d \theta$ y $d / d \varphi$ sobre $\tau_{N-1}(\theta)$ y $\tau_{M-1}(\varphi)$ respectivamente. Estas matrices deben tener la forma genérica dada en (3.23), con nodos en principio arbitrarios en cada una de las variables, manteniendo sus rangos correspondientes: $0<\theta \leq \pi$ $-\pi<\varphi \leq \pi$. Sin embargo, fisicamente se requiere que $-i d / d \varphi$ represente la componente $L_{z}$ del impulso angular, por lo que los nodos sobre $\varphi$ deben ser seleccionados en la forma (3.24) para que se mantenga la relación (3.28), que es la proyección de dicha regla cuántica. Los nodos en la variable $\theta$ quedan libres. Más aún, la misma representación finita de la derivada $d / d \theta$ puede ser seleccionada de otra forma (ver por ejemplo [11]), pero esto será discutido más adelante.

Así, tomemos $N$ puntos distintos $\theta_{j}$ de $(0, \pi)$ para construír la matriz $\tilde{D}_{\theta}$ de acuerdo a (3.23) y la matriz derivada en la variable $\varphi$ queda como $\tilde{D}_{\theta}^{0}$. El hecho de que $Y_{n}^{m}(\theta, \varphi)$ están definidos para índices enteros, excluye el uso de representaciones bivaluadas del impulso angular y nos lleva a pedir que $N$ y $M$ sean números impares.

En la sección 2.2 hemos visto cómo hacer una proyección en el caso de varias variables, por lo que el problema de eigenvalores de $L^{2}$,

$$
\frac{\partial^{2} f}{\partial^{2} \theta}+\cot \theta \frac{\partial f}{\partial \theta}+\frac{1}{\sin ^{2} \theta} \frac{\partial^{2} f}{\partial^{2} \varphi}=-\lambda f=-n(n+1) f
$$

con $f(\theta, \varphi)=Y_{n}^{m}(\theta, \varphi)$, toma la forma matricial

$$
\mathbf{T L}^{2} \mathbf{T}^{-1} \mathbf{f}_{q}^{*}=\lambda_{q}^{*} \mathbf{f}_{q}^{*}
$$

donde $q=1,2 \cdots, N M, \mathrm{~L}^{2}$ es la matriz $N M \times N M$ dada por

$$
\mathbf{L}^{2}=-\left[\mathbf{D}_{\boldsymbol{\theta}}^{2}+\cot \Theta \mathbf{D}_{\boldsymbol{\theta}}+\sin ^{-2} \Theta \mathbf{D}_{\varphi}^{2}\right] .
$$

Aquí, $\mathbf{f}_{q}^{*}$ y $\lambda_{q}^{*}$ son las proyecciones de los eigenvectores y eigenvalores exactos de $L^{2}$, respectivamente y $\mathbf{T}$ es una matriz de similitud dada por $\mathbf{T}=T_{\varphi} \otimes T_{\theta}$. Nótese que (3.41) puede reescribirse como

$$
\mathbf{L}^{2} \mathbf{g}_{q}^{*}=\lambda_{q}^{*} \mathbf{g}_{q}^{*}
$$

con $\mathbf{g}_{q}^{*}=\mathbf{T}^{-1} \mathbf{f}_{q}^{*}$, y ya que el efecto de $\mathbf{T}$ solamente es cancelar un factor de la forma $(-1)^{j+k}$ en los resultados asintóticos [ver por ejemplo la sección 2.2 y las ecuaciones (3.22) y (3.25)], puede ser ignorada y tomar a (3.43) como proyección de (3.40). Las matrices del lado derecho de (3.42) están dadas por

$$
\Theta=I_{M} \otimes \Theta, \quad \mathbf{D}_{\boldsymbol{\theta}}=I_{M} \otimes D_{\theta}, \quad \mathbf{D}_{\varphi}=D_{\varphi}^{0} \otimes I_{N},
$$

donde $\Theta$ es una matriz diagonal con entradas $\Theta_{j k}=\theta_{j} \delta_{j k}, I_{M}\left(I_{N}\right)$ es la matriz identidad de dimensión $M(N)$. Así (3.42) puede reescribirse como

$$
-\mathbf{L}^{2}=I_{M} \otimes\left[D_{\theta}^{2}+\cot \Theta D_{\theta}\right]+\left(D_{\varphi}^{0}\right)^{2} \otimes \sin ^{-2} \Theta .
$$


Ya que $Y_{n}^{m}(\theta, \varphi)$ es el producto (tensorial) de las funciones asociadas de Legendre $P_{n}^{m}(\theta)$ $\operatorname{con} e^{i m \theta}, \mathrm{y}$ tomando en cuenta que el grado máximo del polinomio que puede diferenciarse según (3.23) produciendo valores exactos en los $N$ nodos de $\theta$ (en los $M$ nodos de $\varphi$ ) es $(N-1) / 2[o(M-1) / 2]$, la fórmula (3.41) [o (3.43)] reproduce exactamente los primeros

$$
\sum_{l=0}^{(N-1) / 2}(2 l+1)=\left(\frac{N+1}{2}\right)^{2}
$$

eigenvalores y a sus correspondientes eigenfunciones (no normalizadas) $Y_{n}^{m}(\theta, \varphi)$ evaluadas en los nodos $\left(\theta_{j}, \varphi_{k}\right)$, siempre que $M \geq N$. En este caso $\mathbf{L}^{2}$ tendrá necesariamente $(N+1)^{2} / 4$ eigenvalores reales dados por

$$
\lambda_{q}^{*}=n(n+1),
$$

ordenados de acuerdo a

$$
q=n^{2}+n+m+1, \quad n=0,1, \cdots,(N-1) / 2, \quad m=-n,-n+1, \cdots, n-1, n .
$$

El eigenvector $\mathrm{f}_{q}^{*}$, que corresponde a $\lambda_{q}^{*}$, tiene componentes $f_{s q}^{*}$ dados por

$$
f_{s q}^{*}=c_{n m} P_{n}^{m}\left(\theta_{j}\right) e^{i m k \epsilon},
$$

donde $\epsilon=2 \pi / M, c_{n m}$ es un factor de normalización, $\theta_{j} \in(0, \pi)$, $q$ sigue el mismo orden que en $\lambda_{q}^{*}$, y la relación entre $s, j$ y $k$, está dada por $s=j+(k-1) N$. Es posible escoger los nodos en $\theta$ de tal forma que $\mathbf{L}^{2}$ sea una matriz positiva semidefinida. Para ver esto, nótese que $-\left(D_{\varphi}^{0}\right)^{2}$ es positiva semidefinida ( $D_{\varphi}^{0}$ es antisimétrica) mientras que $\sin ^{-2}(\Theta)$ es positiva definida ya que $\theta_{j} \in(0, \pi), j=1,2, \cdots, N$. Por lo tanto, de acuerdo a (3.45), sólo tenemos que encontrar las condiciones bajo las cuales

$$
L_{\theta}^{2}=-D_{\theta}^{2}-\cot \Theta D_{\theta}
$$

sea positiva semidefinida. Para esto, separemos la diagonal principal de $D_{\theta}$ escribiendo

$$
D_{\theta}=D_{\theta}^{0}+d,
$$

donde la matriz $d$ tiene la diagonal principal de $D_{\theta}$. Así

$$
L_{\theta}^{2}=-\left[\left(D_{\theta}^{0}\right)^{2}+D_{\theta}^{0} d+d D_{\theta}^{0}+d^{2}+\cot \Theta D_{\theta}^{0}+\cot \Theta d\right] .
$$

Por lo tanto, si podemos encontrar puntos $\theta_{j}$ tales que $d=-\cot (\Theta) / 2$, i.e.,

$$
\sum_{l=1}^{N} \cot \frac{\left(\theta_{j}-\theta_{l}\right)}{2}=-\cot \theta_{j}
$$

[cf. (2.10)], la ecuación (3.52) toma la forma

$$
L_{\theta}^{2}=-\left(D_{\theta}^{0}\right)^{2}+d D_{\theta}^{0}-D_{\theta}^{0} d+d^{2}=\left(-D_{\theta}^{0}+d\right)\left(D_{\theta}^{0}+d\right)=D_{\theta}^{t} D_{\theta},
$$


donde el índice $t$ significa transpuesta. Por lo tanto, si los nodos $\theta_{j}$ satisfacen (3.53), $\mathrm{L}^{2}$ es positiva semidefinida. Ahora tenemos que mostrar que (3.53) tiene solución. Para esto, nótese que cot $x$ es la derivada logarítmica de $\sin x$ y que la ecuación (3.53) es la condición de punto crítico para la función de $N$ variables

$$
U(z)=U\left(z_{1}, z_{2}, \cdots, z_{N}\right)=\prod_{k=1}^{N} \sin \left(z_{k}\right) \prod_{i>j}^{N} \sin \frac{\left(z_{i}-z_{j}\right)}{2},
$$

donde $0 \leq z_{j} \leq \pi, j=1,2, \cdots, N$. La existencia (y unicidad) de la solución puede probarse siguiendo las ideas dadas en [46]. Por lo tanto, con esta selección de nodos en la variable $\theta$, la matriz $\mathbf{L}^{2}$ es positiva semidefinida, condición necesaria para cualquier esquema de proyección desde el punto de vista de la Física.

\subsection{Otra matriz para $L^{2}$}

En [11] se obtiene una representación dimensionalmente finita de la derivada para polinomios trigonométricos con paridad definida que puede usarse en substitución de $D_{\boldsymbol{\theta}}$ para construír una matriz para $L^{2}$ con un grado de aproximación más alto como se muestra enseguida. El costo de esto es que no puede hablarse de $u n a$ matriz para $d / d \theta$ que pueda ser substituída directamente en (3.50), a menos que se acepte una pérdida en la precisión de los resultados (ver [11]). La representación de $d / d \theta$ (salvo por una transformación de similitud) es en este caso una matriz que será denotada por $D_{\theta}^{*}$, construída con $N$ puntos arbitrarios $\theta_{j} \in(0, \pi)$ por medio de una fórmula similar a (3.23)

$$
\left(D_{\theta}^{*}\right)_{i j}= \begin{cases}\sum_{l=1}^{N} \cot \left(\theta_{i}-\theta_{l}\right), & i=j, \\ \cot \left(\theta_{i}-\theta_{j}\right), & i \neq j .\end{cases}
$$

La forma que $L_{\theta}^{2}$ adopta en este case es

$$
L_{\theta}^{2}=-\left(D_{\theta}^{*}\right)^{2}-\cot \Theta D_{\theta}^{*}+N O,
$$

donde $\Theta$ es nuevamente una matriz diagonal con entradas $\Theta_{j k}=\theta_{j} \delta_{j k}$ y $O$ es una matriz de proyección con 1's en cada entrada, i.e., $O_{j k}=1$. El grado de aproximación de (3.57) es más alto que el de (3.50). Mientras que la primera matriz produce resultados exactos en $N$ nodos ( $N$ par o impar) para polinomios trigonométricos (de paridad definida) hasta de grado $N$, la segunda produce resultados exactos para polinomios de grado hasta $(N-1) / 2$ ( $N$ impar). Por lo tanto, el uso de (3.57) en (3.45) da la matriz

$$
-\mathbf{L}^{2}=I_{M} \otimes\left[\left(D_{\theta}^{*}\right)^{2}+\cot \Theta D_{\theta}^{*}-N O\right]+\left(D_{\varphi}^{0}\right)^{2} \otimes \sin ^{-2} \Theta .
$$


con un grado más alto de aproximación: si $N$ es impar y $M=2 N+1$, entonces (3.58) reproduce los primeros

$$
\sum_{l=0}^{N}(2 l+1)=(N+1)^{2}
$$

eigenvalores y eigenvectores exactos, mientras que (3.45) sólo da los primeros $(N+1)^{2} / 4$ para los mismos valores de $N$ y $M$. Sin embargo, a pesar de que esta representación de $\mathbf{L}^{2}$ tiene un grado de aproximación más alto que (3.45), no puede asociarse al subgrupo de rotaciones discretas de la sección 3.1, por lo que sólo tiene aplicación para calcular numéricamente soluciones de problemas cuánticos, como se verá más adelante.

\subsection{Las relaciones de conmutación}

Hasta ahora hemos encontrado las proyecciones de $L^{2}$ y de $L_{z}$ sobre un espacio lineal de dimensión finita, pero no hemos dicho nada sobre cómo se proyectan las otras componentes del impulso angular $\vec{L}$, ni el álgebra asociada a todas ellas. En esta sección encontraremos las proyecciones de las componentes restantes y de las relaciones de conmutación de estos operadores.

Primeramente calcularemos la forma discreta de las componentes $L_{x}, L_{y}$ y $L_{z}$ de $\vec{L}$ en términos de las variables esféricas $\theta, \varphi$ y de sus derivadas usando las fórmulas

$$
\begin{aligned}
& L_{x}=i\left(\sin \phi \frac{\partial}{\partial \theta}+\cos \phi \cot \theta \frac{\partial}{\partial \varphi}\right) \\
& L_{y}=-i\left(\cos \phi \frac{\partial}{\partial \theta}+\sin \phi \cot \theta \frac{\partial}{\partial \varphi}\right) \\
& L_{z}=-i \frac{\partial}{\partial \varphi}
\end{aligned}
$$

y mostraremos que el método de proyección usado en este trabajo produce resultados consistentes al calcular el cuadrado del impulso angular por medio de (3.42), o bien, usando la expresión

$$
L^{2}=L_{x}^{2}+L_{y}^{2}+L_{z}^{2}
$$

Este hecho no resulta ser obvio, ya que las representaciones matriciales obtenidas a partir de (3.60) no necesariamente deben satisfacer una relación obtenida diferencialmente.

De acuerdo al método de discretización descrito en la sección 3.2, seleccionaremos $K$ nodos $^{1}$ en la variable $\theta$ y $M$ en $\varphi$ para construír las matrices diagonales $\Theta$ y $\Phi$ respectivamente, así como las matices $D_{\theta}$ y $D_{\varphi}$, representaciones de las derivadas $d / d \theta$ y $d / d \varphi$. Así tenemos que las proyecciones de $L_{x}, L_{y}$ y $L_{z}$ son las matrices

$$
\mathrm{L}_{x}=i\left(\sin \Phi \otimes D_{\theta}+\cos \Phi D_{\varphi} \otimes \cot \Theta\right),
$$

\footnotetext{
${ }^{1}$ Por conveniencia, de aquí en adelante, usaremos el índice $K$ para denotar el número de nodos en la coordenada $\theta$.
} 


$$
\begin{aligned}
& \mathbf{L}_{y}=-i\left(\cos \Phi \otimes D_{\theta}-\sin \Phi D_{\varphi} \otimes \cot \Theta\right) \\
& \mathbf{L}_{z}=-i D_{\varphi} \otimes I_{K} .
\end{aligned}
$$

Comencemos por calcular los conmutadores $\left[D_{\varphi}, \sin \Phi\right]$ y $\left[D_{\varphi}, \cos \Phi\right]$. Usando algunas relaciones trigonométricas, obtenemos

$$
D_{\varphi} \sin \Phi=\sin \Phi D_{\varphi}+\cos \Phi-U_{c}
$$

y

$$
D_{\varphi} \cos \Phi=\cos \Phi D_{\varphi}-\sin \Phi+U_{s},
$$

donde $U_{c}$ y $U_{s}$ son matrices de proyección definidas por

$$
\left(U_{c}\right)_{j k}=(-1)^{j+k} \cos \left(\frac{\varphi_{j}+\varphi_{k}}{2}\right), \quad\left(U_{s}\right)_{j k}=(-1)^{j+k} \sin \left(\frac{\varphi_{j}+\varphi_{k}}{2}\right) .
$$

Estas matrices pueden sumarse para formar la matriz compleja $U=U_{c}+i U_{s}$ que proyecta el polinomio trigonométrico

$$
f_{m}(\varphi)=\sum_{l=-m}^{m} c_{l} e^{i l \varphi}, \quad m \leq m^{*}=(M-1) / 2,
$$

en las componentes de más alto grado $\left|m^{*}\right|$. Para aclarar este punto, tomemos los polinomios $\cos \varphi f_{m}(\varphi), \sin \varphi f_{m}(\varphi)$, y calculemos sus derivadas usando $D_{\varphi}$. Ya que el uso de $D_{\varphi}$ produce resultados exactos para polinomios de grado $m^{*}$ a lo más, evaluados en los nodos, las fórmulas

$$
D_{\varphi} \sin \Phi f_{m}=\sin \Phi D_{\varphi} f_{m}+\cos \Phi f_{m} \quad D_{\varphi} \cos \Phi f_{m}=\cos \Phi D_{\varphi} f_{m}-\sin \Phi f_{m}
$$

son válidas siempre que $m \leq m^{*}-1$. Aquí, $f_{m}$ es el vector formado con los valores de $f_{m}\left(\varphi_{j}\right)$. Por lo tanto, comparando estas expresiones con (3.63) y (3.64), nuestra afirmación acerca de $U$ sobre su carácter de matriz de proyección, se sigue inmediatamente. Calculemos ahora la suma $\mathbf{L}_{x}^{2}+\mathbf{L}_{y}^{2}$. De (3.62) se obtiene que

$$
\begin{aligned}
-\left(\mathbf{L}_{x}^{2}+\mathbf{L}_{y}^{2}\right) & =\mathbf{D}_{\theta}^{2}+\left(\cos \Phi D_{\varphi} \sin \Phi-\sin \Phi D_{\varphi} \cos \Phi\right) \otimes \cot \Theta D_{\theta} \\
& +\left(\cos \Phi D_{\varphi} \cos \Phi+\sin \Phi D_{\varphi} \sin \Phi\right) D_{\varphi} \otimes \cot ^{2} \Theta .
\end{aligned}
$$

Definamos la matriz $\mathbf{L}^{\prime 2}$ como la matriz obtenida mediante la suma de los cuadrados de las componentes de $\overrightarrow{\mathbf{L}}$, es decir, $\mathbf{L}^{\prime 2}=\mathbf{L}_{x}^{2}+\mathbf{L}_{y}^{2}+\mathbf{L}_{z}^{2}$. La substitución de (3.63) y (3.64) en (3.68) produce

$$
-\mathbf{L}^{\prime 2}=-\mathbf{L}^{2}-U_{c}^{\prime} \otimes \cot \Theta D_{\theta}+U_{s}^{\prime} D_{\varphi} \otimes \cot ^{2} \Theta
$$

donde $\mathbf{L}^{2}$ está dada por (3.42) y

$$
U_{c}^{\prime}=\cos \Phi U_{c}+\sin \Phi U_{s}, \quad U_{s}^{\prime}=\cos \Phi U_{s}-\sin \Phi U_{c} .
$$


Puesto que las componentes de estas dos matrices están dadas por

$$
\left(U_{c}^{\prime}\right)_{j k}=(-1)^{j+k} \cos \left(\frac{\varphi_{j}-\varphi_{k}}{2}\right), \quad\left(U_{s}^{\prime}\right)_{j k}=(-1)^{j+k+1} \sin \left(\frac{\varphi_{j}-\varphi_{k}}{2}\right),
$$

y los nodos $\varphi_{j}$ están simetricamente dispuestos en $[-\pi, \pi], U_{c}^{\prime}$ y $U_{s}^{\prime}$ resultan ser el producto de una matriz de permutación por $U_{c}$ y $U_{s}$, respectivamente. Por lo tanto, $\mathbf{L}^{2}$ y $\left(\mathbf{L}^{\prime}\right)^{2}$ difieren sólo por matrices que proyectan un vector sobre las componentes polinomiales de grado más alto, y producen los mismos resultados cuando se aplican a vectores formados por productos de polinomios en $\theta$ y $\varphi$ de grados $(K-1) / 2$ y $(M-3) / 2$ a lo más, respectivamente, evaluados en los nodos. En otras palabras, la dimensión del espacio lineal en donde el uso de $\left(\mathbf{L}^{\prime}\right)^{2}$ produce resultados exactos, es más pequeña que la dimensión del espacio correspondiente a $\mathbf{L}^{2}$, sin embargo, esta diferencia puede compensarse si se incrementa $M$. El hecho de que $\left(\mathbf{L}^{\prime}\right)^{2}$ y $\mathbf{L}^{2}$ no sean iguales, se debe fundamentalmente a que la derivada discreta de las funciones circulares cos y sin coinciden exactamente con sus contrapartes continuas sólo cuando nos restringimos a un subespacio de menor dimensión [cf. (3.63) y (3.64)].

Ahora encontremos la forma de los conmutadores de las componentes $L_{x}, L_{y}$ y $L_{z}$ en el espacio de proyección. Para esto usaremos las expresiones de estas componentes en coordenadas cartesianas, sobre las cuales tomaremos $N$ ceros de Hermite sobre el eje $x$, $M$ sobre $y$ y $K$ sobre $z$ para formar el conjunto de nodos cartesianos $\left(x_{n}, y_{m}, z_{k}\right)$, y las matrices diagonales que representan a las variables $x, y$ y $z$,

$$
\begin{aligned}
& \mathbf{X}=\left(I_{K} \otimes I_{M} \otimes X\right), \\
& \mathbf{Y}=\left(I_{K} \otimes Y \otimes I_{N}\right), \\
& \mathbf{Z}=\left(Z \otimes I_{M} \otimes I_{N}\right) .
\end{aligned}
$$

Las diagonales principales de $X, Y$ y $Z$, contienen los conjuntos de nodos $x_{n}, y_{m}$ y $z_{k}$, respectivamente. Tomando en cuenta (2.14), las representaciones de las derivadas parciales $\partial / \partial x, \partial / \partial y$ y $\partial / \partial z$, son las matrices $N M K \times N M K$

$$
\begin{aligned}
& \mathbf{D}_{x}=\left(I_{K} \otimes I_{M} \otimes \tilde{D_{x}^{0}}\right), \\
& \mathbf{D}_{y}=\left(I_{K} \otimes \tilde{D}_{y}^{0} \otimes I_{N}\right), \\
& \mathbf{D}_{z}=\left(\tilde{D_{z}^{0}} \otimes I_{M} \otimes I_{N}\right) .
\end{aligned}
$$

Por lo tanto, las componentes matriciales del impulso angular en coordenadas cartesianas (con $\hbar=1$ ) toman la forma

$$
\begin{aligned}
& \mathbf{L}_{x}=-i\left(\mathbf{Y D}_{z}-\mathbf{Z D}_{y}\right), \\
& \mathbf{L}_{y}=-i\left(\mathbf{Z D}_{x}-\mathbf{X D}_{z}\right), \\
& \mathbf{L}_{z}=-i\left(\mathbf{X D}_{y}-\mathbf{Y D}_{x}\right),
\end{aligned}
$$

y usando (2.14), (2.15) y (2.30) puede obtenerse facilmente que los conmutadores están dados por

$$
\left[\mathbf{L}_{x}, \mathbf{L}_{y}\right]=i \mathbf{L}_{z}-U_{z} \otimes\left(D_{y} \otimes X-Y \otimes D_{x}\right)
$$




$$
\begin{aligned}
& {\left[\mathbf{L}_{y}, \mathbf{L}_{z}\right]=i \mathbf{L}_{x}-\left(D_{z} \otimes Y-Z \otimes D_{y}\right) \otimes U_{x},} \\
& {\left[\mathbf{L}_{z}, \mathbf{L}_{x}\right]=i \mathbf{L}_{y}-\left(Z \otimes U_{y} \otimes D_{x}-D_{z} \otimes U_{y} \otimes X\right),}
\end{aligned}
$$

donde $U_{j}=S_{j} O_{j} S_{j}^{-1}$ (el índice $j$ indica $x, y$ o $z$ ), $O_{j}$ es la matriz de proyección cuyas componentes son todas iguales a 1 [cf. (2.30)]. En la discusión que sigue a la ecuación (2.30) se mostró que la matriz $U_{j}$ proyecta los vectores del subespacio de funciones del tipo dado por (2.20), sobre la componente polinomial (en la variable correspondiente) de grado más alto, haciendo que (3.75) tome la forma esperada

$$
\begin{aligned}
& {\left[\mathbf{L}_{x}, \mathbf{L}_{y}\right]=i \mathbf{L}_{z},} \\
& {\left[\mathbf{L}_{y}, \mathbf{L}_{z}\right]=i \mathbf{L}_{x},} \\
& {\left[\mathbf{L}_{z}, \mathbf{L}_{x}\right]=i \mathbf{L}_{y},}
\end{aligned}
$$

siempre que estas matrices se apliquen sobre cualquier vector del subespacio de productos tensoriales de funciones de la forma (2.20) de grado a lo más $N-2$ en $x, M-2$ en y y $K-2$ en $z$. 


\section{Capítulo 4}

\section{La ecuación de Schrödinger discreta}

\subsection{Preliminares}

Hemos mencionado ya que el método de discretización establecido en los capítulos anteriores, además de preservar las forma de las reglas cuánticas entre las distintas componentes del impulso y las de la posición, así como las de conmutación, genera matrices finitas para las ecuaciones de onda, produciendo resultados convergentes a los continuos, siempre que los nodos con que se construyen dichas matrices se obtengan a partir de ciertas ecuaciones en las que el potencial juega un papel principal, haciendo que la configuración de los nodos dependa del potencial que intervenga en la ecuación de onda. En este capítulo tendremos ocasión de corroborar estas afirmaciones y de dar credibilidad a esta técnica de discretización como tal y como método numérico, al discretizar la ecuación de Schrödinger independiente del tiempo para el átomo de Hidrógeno y encontrar sus soluciones discretas. Consideraremos primeramente el potencial de Coulomb y después las correcciones de estructura fina. Finalmente trataremos el caso importante del átomo de Hidrógeno en un campo magnético uniforme de intensidad arbitraria.

Hemos seleccionado estos problemas porque cumplen con los requisitos necesarios para mostrar las ventajas del método propuesto: son problemas de calificación para todo procedimiento de discretización (un método que no pueda resolver numericamente al menos los dos primeros problemas, no puede ser considerado seriamente como método numérico, mucho menos como método de discretización para la Mecánica Cuántica). El último caso se distingue entre los otros, primero porque a pesar de ser tan viejo, no había tenido una solución general para todo el rango del parámetro que define al campo magnético y resulta un problema importante en Astrofísica, Estado Sólido y Espectroscopía Atómica. Sólo recientemente [37] se ha dado un método de solución para valores arbitrarios del campo magnético. Por otra parte, es un problema de valores a la frontera no separable en las coordenadas y altamente singular por lo que nuestro método requerirá una modificación que se presentará en este mismo capítulo, que, dicho sea de paso, se encuentra más orientado hacia aspectos de cálculo numérico. 


\subsection{El átomo de Hidrógeno}

En ésta y en las siguientes secciones, tomaremos $\hbar=1 \mathrm{y}$ como unidades de longitud y energía al radio de Bohr $a_{0}=\hbar^{2} / m_{e} e^{2}=5.3 \times 10^{-9} \mathrm{~cm}$ y al doble de un Rydberg $m_{e} e^{4} / \hbar^{2}=27.2 \mathrm{eV}$, respectivamente.

De acuerdo a las ideas de la sección 2.1, para obtener una forma matricial apropiada de la ecuación de Schrödinger para el electrón en un campo de Coulomb

$$
\left[-\frac{1}{2 r^{2}} \frac{\partial}{\partial r}\left(r^{2} \frac{\partial}{\partial r}\right)+\frac{1}{2 r^{2}} L^{2}-\frac{1}{r}\right] \psi(r, \theta, \varphi)=E \psi(r, \theta, \varphi),
$$

es necesario extraer de $\psi(r, \theta, \varphi)$ el comportamiento singular en los puntos extremos de los intervalos (ver también [8], [16] y [19]). Esto puede hacerse en parte por medio del cambio de variable

$$
\psi(r, \theta, \varphi)=\psi_{\infty}(r, \theta, \varphi) f(r, \theta, \varphi)
$$

donde $\psi_{\infty}(r, \theta, \varphi)$ es la solución de (4.1) para estados ligados cuando $r \rightarrow \infty$, dada por

$$
\psi_{\infty}(r, \theta, \varphi)=e^{-\sqrt{2 \varepsilon} r}
$$

La energía se escribe aquí como $-\varepsilon$ pues será usada en los cálculos numéricos como un parámetro de prueba.

La substitución de (4.2) y (4.3) en (4.1) produce

$$
\left[-\frac{1}{2} \frac{\partial^{2}}{\partial r^{2}}-\left(\frac{1}{r}-\sqrt{2 \varepsilon}\right) \frac{\partial}{\partial r}+\frac{1}{2 r^{2}} L^{2}-\left(\frac{1-\sqrt{2 \varepsilon}}{r}\right)-\varepsilon\right] f(r, \theta, \varphi)=E f(r, \theta, \varphi) .
$$

Ya que $f(r, \theta, \varphi)$ puede escribirse como un producto de funciones univariadas de $r, \theta$ $\mathrm{y} \varphi$, podemos proyectar cada una de tales funciones sobre subespacios de polinomios para generar representaciones matriciales de dimensión finita de las derivadas parciales (ver secciones 2.2, 3.2 y el Apéndice B). Así, a la proyección en las variables angulares (descrita en la sección 3.2) sobre el subespacio $\tau_{M-1}(\varphi) \otimes \tau_{K-1}(\theta)$, tenemos que añadir la proyección de la función radial sobre el subespacio de polinomios $\pi_{N-1}(r)$. Esto produce el espacio tensorial de proyección $U_{\tilde{N}}(r, \theta, \varphi)=\tau_{M-1}(\varphi) \otimes \tau_{K-1}(\theta) \otimes \pi_{N-1}(r)$ de dimensión $N^{*}=M K N$ en el cual la forma matricial de (4.4) está dada por

$$
\mathrm{HF}_{q}^{*}=E_{q}^{*} \mathbf{F}_{q}^{*}, \quad s=1,2, \cdots, N^{*},
$$

donde el Hamiltoniano discreto es

$$
\mathbf{H}=-\frac{1}{2} \mathbf{D}_{r}^{2}-\left(\mathbf{r}^{-1}-\sqrt{2 \varepsilon} \mathbf{1}\right) \mathbf{D}_{r}+\frac{1}{2} \mathbf{r}^{-2} \mathbf{L}^{2}-(1-\sqrt{2 \varepsilon}) \mathbf{r}^{-1}-\varepsilon \mathbf{1}
$$

Aquí, $\mathrm{D}_{r}$ es el producto tensorial

$$
\mathrm{D}_{r}=1_{M} \otimes 1_{K} \otimes \tilde{D}_{r}
$$


donde $\tilde{D}_{r}$ es la representación de la derivada radial que tiene la estructura (2.5) y la matriz diagonal $\mathbf{r}$ está definida por

$$
\mathbf{r}=1_{M} \otimes 1_{K} \otimes R .
$$

La matriz diagonal $R$ tiene los nodos radiales $r_{j}, j=1,2, \cdots, N$, sobre su diagonal principal. $\mathrm{L}^{2}$ está dado por (3.42) y 1 es la matriz identidad de dimensión $N^{*}$. Ya que la parte de $f(r, \theta, \varphi)$ que depende de $r$ son polinomios (polinomios de Laguerre multiplicados por una potencia de $r$ ) y la dimensión de $\pi_{N-1}(r)$ es $N$, se podría esperar un conjunto de $N(K+1)^{2} / 4$ eigenvectores $\mathbf{F}_{q}^{*}$ que coincidan exactamente en los nodos con las primeras eigenfunciones del átomo de Hidrógeno evaluadas en esos puntos (siempre que $M \geq K$ ). Sin embargo, debido al hecho de que el argumento de la función radial depende del número cuántico principal $n$, el cual está relacionado al índice que define el grado $n_{L}$ de la propia función radial (el polinomio de Laguerrre) y al número cuántico azimutal $l$ a través de $n=n_{L}+l+1$, la proyección de la parte radial de (4.6) para los estados $s$ sobre el espacio de proyección $\pi_{N-1}(r)$, es decir, la ecuación de eigenvalores

$$
H_{r} f_{j}^{*}=E_{j}^{*} f_{j}^{*}, \quad j=1,2, \cdots, N
$$

donde

$$
H_{r}=-\frac{1}{2} \tilde{D}_{r}{ }^{2}-\left(R^{-1}-\sqrt{2 \varepsilon} 1_{N}\right) \tilde{D}_{r}-(1-\sqrt{2 \varepsilon}) R^{-1}-\varepsilon 1_{N}
$$

reproducirá sólo un eigenvalor exacto: el que corresponde al caso en que $\varepsilon=-E=1 /\left(2 n^{2}\right)$ para algún $n \leq N$. Como consecuencia, el número de energías exactas dadas por $(4.5)$ está dado por la bien conocida fórmula

$$
\sum_{l=0}^{n-1}(2 l+1)=n^{2}
$$

siempre que $n \leq n_{\max }$ para algún $n_{\max }$ que enseguida determinamos. Ya que $\tilde{D}_{r}$ da derivadas exactas de polinomios de grado $N-1$ a lo más y la parte radial de $f(r, \theta, \varphi)$ es un polinomio de grado $n-1=n_{L}+l$, se tiene que $n_{\max }=N$, y por lo tanto, el método produce el número correcto de eigenvalores exactos correspondientes al multiplete definido por $n$ siempre que $n \leq N$ y $M \geq K \geq 2 N-1$. Sin embargo, es necesario escoger los nodos radiales en tal forma que

$$
\sum_{l=1}^{N} \frac{1}{\left(r_{j}-r_{l}\right)}=\sqrt{2 \varepsilon}-\frac{1}{r_{j}}, \quad j=1,2, \cdots, N
$$

para que la matriz $H_{r}$ de (4.10) sea hermitiana [cf. (2.10) y la discusión que le sigue]. La solución de (4.12) puede encontrarse fácilmente en términos de los ceros de Laguerre (ver [49]). Así, estos puntos coinciden con los $N$ ceros del polinomio de Laguerre $L_{N}^{(1)}(2 \sqrt{2 \varepsilon} r)$ y producen una matrix positiva definida $\mathbf{r}^{-1}$ en (4.6) y una matriz hermitiana para $H_{r}$ (salvo por una transformación de similitud). Esto último puede probarse si separamos la 
diagonal principal de $\tilde{D}_{r}$ escribiendo $\tilde{D}_{r}=Q D_{r}^{0} Q^{-1}+d[$ ver (2.5)] y usando (4.12) para obtener

$$
H_{r}=Q\left[\frac{1}{2}\left(-\left(D_{r}^{0}\right)^{2}+d D_{r}^{0}-D_{r}^{0} d+d^{2}\right)+V\right] Q^{-1}=Q\left(\frac{1}{2} D_{r}^{t} D_{r}+V\right) Q^{-1},
$$

donde $V$ es la matriz diagonal

$$
V=-(1-\sqrt{2 \varepsilon}) R^{-1}-\varepsilon 1_{N} .
$$

Por lo tanto, la parte radial de $\mathbf{H}_{r}$ de (4.6) también tiene eigenvalores reales ya que

$$
\mathbf{H}_{r}=1_{M} \otimes 1_{K} \otimes H_{r} .
$$

Sin embargo, debido a la arbitrariedad de los nodos en $\theta$, pueden encontrarse algunos eigenvalores complejos en el espectro de (4.5). Para excluirlos, podemos fijar estos puntos por medio de la condición (3.53) para dar una matriz semipositiva definida $\mathrm{L}^{2}$ (salvo también por una transformación de similitud). Es importante repetir que tales transformaciones no son esenciales para un problema de eigenvalores como (4.5) puesto que las matrices de similitud pueden agruparse en una sola matriz diagonal de $N^{*} \times N^{*} \mathbf{S}$ y escribir (4.5) en la forma

$$
\mathbf{H}^{H} \mathbf{g}_{q}=E_{q}^{*} \mathbf{g}_{q}, \quad q=1,2, \cdots, N^{*},
$$

donde $\mathbf{H}^{H}$ es hermitiana y $\mathbf{g}_{q}=\mathbf{S}^{-1} \mathbf{F}_{s}^{*}$.

Resumiendo, si los nodos $\left(r_{i}, \theta_{j}, \varphi_{k}\right)$ se escogen de tal forma que $r_{i}, i=1, \cdots, N$, satisfacen (4.12), $\theta_{j}, j=1, \cdots, K$, satisfacen (3.53) y $\varphi_{k}=-\pi+2 \pi k / M, k=1, \cdots, M$, la proyección de la ecuación de Schrödinger en $U_{\tilde{N}}(r, \theta, \varphi)$ es la ecuación (4.5), donde $\mathbf{H}$ es una matriz con eigenvalores reales dada por (4.6). El espectro de esta matrix contiene necesariamente las energías de amarre exactas del $n$-ésimo multiplete del átomo de Hidrógeno, siempre que $n \leq N(N \geq 2), M \geq K \geq 2 N-1$ y $\varepsilon=1 /\left(2 n^{2}\right)$. Las eigenfunciones del multiplete divididas por $e^{-\sqrt{2 \varepsilon r}}$ [cf. (4.2)-(4.3)] se determinan también exactamente en los nodos (salvo por un factor de normalización), ya que las matrices que representan a las derivadas parciales construídas con estos nodos, producen resultados exactos para polinomios de la forma

$$
\rho(r) P_{l}^{m}(\theta) e^{i m \varphi},
$$

donde $\rho(r)$ es un polinomio de grado $N-1$ a lo más, $l=0,1, \cdots,(K-1) / 2$ y $m=-l, \cdots, l$. Además de estos eigenvalores exactos, el número correcto de valores que aproximan a la energía de algún otro multiplete definido por otro índice $n^{\prime}$ menor o igual que $N$, también aparecen en el espectro de (4.5). Como era de esperarse, estos valores aproximados convergen numericamente a los valores exactos de los primeros $(K-1) / 2$ multipletes $(M \geq K \geq 2 N-1)$ cuando $N$ crece.

Debe decirse que en el espectro de (4.5) aparece el cero y valores positivos. De hecho, el número de valores no negativos es mayor que el número de valores negativos, y en principio pueden interpretarse como energías correspondientes a estados libres, pues numericamente se observa que a medida que $N$ crece, crece el número de eigenvalores no negativos y 
se distribuyen a lo largo de $(0, \infty)$ aunque sin convergencia a valores específicos.

Mostraremos ahora algunos resultados numéricos. En la Tabla I mostramos las energías de amarre de los primeros cuatro multipletes obtenidas diagonalizando (4.5) con $N=4$ y $M=K=7$.

TABLA I. Algunos eigenvalores negativos de Eq. (4.5) con $\mathbf{H}$ dado por (4.6) para $N=4$, $M=K=7$ y $\varepsilon_{n}=1 /\left(2 n^{2}\right)$ con $n=1,2,3,4$. Para distinguir facilmente la energía exacta del $n$-ésimo multiplete, se muestra el valor $-1 / \sqrt{2 E_{s}^{*}}$.

\begin{tabular}{|c|c|c|c|}
\hline 1.00000000000000 & 1.00215707302795 & 1.03168585750231 & 1.08896461090157 \\
\hline 2.04388882694316 & 1.99999999999993 & 2.00129478110128 & 2.00555350797036 \\
\hline 2.04388882694346 & 2.00000000000000 & 2.00129478110130 & 2.00555350797038 \\
\hline 2.04388882694354 & 2.00000000000000 & 2.00129478110132 & 2.00555350797038 \\
\hline \multirow[t]{26}{*}{2.09804764737169} & 2.00000000000000 & 2.00543455491769 & 2.06096113941247 \\
\hline & 3.03156106874861 & 2.99999999999982 & 3.00051376536271 \\
\hline & 3.03156106874867 & 2.99999999999990 & 3.00051376536275 \\
\hline & 3.03156106874879 & 2.99999999999995 & 3.00051376536277 \\
\hline & 3.03156106874883 & 2.99999999999999 & 3.00051376536278 \\
\hline & 3.03156106874884 & 2.99999999999999 & 3.00051376536279 \\
\hline & 3.09880783441467 & 2.99999999999999 & 3.01040973704290 \\
\hline & 3.09880783441493 & 3.00000000000001 & 3.01040973704298 \\
\hline & 3.09880783441499 & 3.00000000000002 & 3.01040973704302 \\
\hline & 3.16392960609153 & 3.00000000000014 & 3.03184205479519 \\
\hline & 5.34059520750606 & 4.06329322772771 & 3.99999999999962 \\
\hline & 5.34059520750857 & 4.06329322772843 & 3.99999999999991 \\
\hline & 5.34059520750915 & 4.06329322772855 & 3.99999999999995 \\
\hline & 5.34059520750938 & 4.06329322772857 & 3.99999999999997 \\
\hline & 5.34059520750947 & 4.06329322772861 & 3.99999999999997 \\
\hline & 5.34059520750989 & 4.06329322772862 & 3.99999999999998 \\
\hline & 5.34059520751064 & 4.06329322772872 & 4.00000000000000 \\
\hline & & 4.25955351771777 & 4.00000000000000 \\
\hline & & 4.25955351771817 & 4.00000000000001 \\
\hline & & 4.25955351771843 & 4.00000000000001 \\
\hline & & 4.25955351771845 & 4.00000000000001 \\
\hline & & 4.25955351771845 & 4.00000000000002 \\
\hline & & 4.70250143548207 & 4.00000000000004 \\
\hline & & 4.70250143548227 & 4.00000000000005 \\
\hline & & 4.70250143548250 & 4.00000000000033 \\
\hline & & & 4.00000000000039 \\
\hline
\end{tabular}


En la Tabla II se muestra la convergencia numérica de los eigenvalores de (4.5) a las energías exactas cuando $N$ crece. La seleción $M=K=7$ produce convergencia sólo para los primeros tres multipletes.

TABLA II. Algunos eigenvalores negativos de (4.5) con $\mathbf{H}$ dado por (4.6) con $M=K=7$ y $\varepsilon=1 / 2$ convergiendo a las energías exactas de los primeros tres multipletes del átomo de Hidrógeno (mostrada aquí como $1 / \sqrt{2 E_{n}}=n, n=1,2,3$ ) cuando $N$ crece. El primer eigenvalor es exacto en todos los casos.

\begin{tabular}{ccccc}
\hline$n$ & $N=5$ & $N=10$ & $N=15$ & $N=20$ \\
\hline 1 & 0.99999999999996 & 1.00000000000005 & 1.00000000000003 & 1.00000000000003 \\
2 & 2.01049276733108 & 2.00000276409036 & 2.00000000023245 & 1.99999999999987 \\
& 2.01049276733135 & 2.00000276409060 & 2.00000000023589 & 2.00000000000002 \\
& 2.01049276733172 & 2.00000276409188 & 2.00000000023606 & 2.00000000000011 \\
& 2.02305632906585 & 2.00000703530320 & 2.00000000063539 & 2.00000000000116 \\
3 & & & & \\
& & 3.01884262920929 & 3.00022234719467 & 3.00000127729828 \\
& & 3.01884262920934 & 3.00022234720154 & 3.00000127729917 \\
& & 3.01884262920941 & 3.00022234720269 & 3.00000127729919 \\
& & 3.01884262921074 & 3.00022234720273 & 3.00000127730065 \\
& & 3.01884262921528 & 3.00022234720280 & 3.00000127730920 \\
& & 3.05122930639900 & 3.00072902585704 & 3.00000472833179 \\
& & 3.05122930639920 & 3.00072902585744 & 3.00000472833209 \\
& & 3.05122930640030 & 3.00072902585783 & 3.00000472833728 \\
& & 3.07657778934264 & 3.00117867702690 & 3.00000812988290 \\
\hline
\end{tabular}

\subsection{Impulso angular total}

De acuerdo al método de discretización presentado en este trabajo, la representación finito-dimensional del impulso angular total del electrón $\vec{J}=\vec{L}+\vec{S}$ es la $2 N^{*} \times 2 N^{*}$ matriz vectorial

$$
\overrightarrow{\mathbf{J}}=I_{2} \otimes \overrightarrow{\mathbf{L}}+\vec{S} \otimes I_{N^{*}},
$$

donde $\overrightarrow{\mathbf{L}}$ es la $N^{*} \times N^{*}$ matriz vectorial que representa al impulso angular cuyas componentes están dadas por (3.74) y $\vec{S}=\vec{\sigma} / 2$ es el impulso angular intrínseco del electrón, dado en términos de las matrices de Pauli $\sigma_{x}, \sigma_{y}$ y $\sigma_{z}$. Entonces, las componentes de $\overrightarrow{\mathbf{L}}$ se pueden escribir en la forma

$$
\begin{aligned}
& \mathbf{L}_{x}=i\left(\sin \Phi \otimes D_{\theta}+\cos \Phi D_{\varphi} \otimes \cot \Theta\right), \\
& \mathbf{L}_{y}=-i\left(\cos \Phi \otimes D_{\theta}-\sin \Phi D_{\varphi} \otimes \cot \Theta\right), \\
& \mathbf{L}_{z}=-i D_{\varphi} \otimes I_{K} .
\end{aligned}
$$


Por lo tanto $\mathbf{J}^{2}$ toma la forma

$$
\mathbf{J}^{2}=\mathbf{J}_{x}^{2}+\mathbf{J}_{y}^{2}+\mathbf{J}_{z}^{2}
$$

donde

$$
\mathbf{J}_{x}=I_{2} \otimes \mathbf{L}_{x}+S_{x} \otimes I_{\tilde{N}}, \quad \mathbf{J}_{y}=I_{2} \otimes \mathbf{L}_{y}+S_{y} \otimes I_{\tilde{N}}, \quad \mathbf{J}_{z}=I_{2} \otimes \mathbf{L}_{z}+S_{z} \otimes I_{\tilde{N}}
$$

El espectro de (4.20) contiene eigenvalores que concuerdan hasta quince dígitos con los valores exactos $j(j+1), j=1 / 2,3 / 2, \cdots l_{\max }-1 / 2, l_{\max }=(K-1) / 2, M \geq K(M$ y $K$ son números impares), como se muestra en la Tabla III para valores pequeños de $K=M$. Nótese que el número total de estados se da en forma correcta. Los eigenvalores que no siguen la sucesión correcta desaparecen cuando $K=M$ se incrementa.

TABLA III. Eigenvalores de (4.20) que siguen la sucesión $j(j+1), 1 / 2 \leq j \leq(K-1) / 2$, con $K=M=3,5,7$. Para distinguirlos facilmente, se muestra el índice $j^{*}=(-1+\sqrt{1+4 \lambda}) / 2$. El dígito entre paréntesis indica el número de eigenvalores iguales repetidos.

\begin{tabular}{cll}
\hline$K=3$ & \multicolumn{1}{c}{$K=5$} & \multicolumn{1}{c}{$K=7$} \\
\hline $0.50000000000000(4)$ & $0.50000000000000(4)$ & $0.50000000000000(4)$ \\
& $1.50000000000000(6)$ & $1.50000000000000(8)$ \\
1.50000000000002 & \\
1.50000000000011 & 2.49999999999997 \\
& & $2.49999999999999(2)$ \\
& & $2.50000000000000(8)$ \\
& & 2.50000000000001 \\
\hline
\end{tabular}

Así, nuestro esquema de proyección produce una representación finita de los operadores de impulso angular que reproduce las soluciones continuas en problemas de eigenvalores espinoriales donde la solución puede escribirse en términos de productos de polinomios trigonométricos por potencias semienteras de ciertas funciones (éste es el caso de las eigenfunciones espinoriales de $J_{z}$ y $J^{2}$ ). Para otros casos, el método requiere tomar en cuenta las singularidades de la ecuación diferencial o una modificación adicional para problemas no separables.

\subsection{Estructura fina del Hidrógeno}

En esta sección consideramos el problema de un electrón clásico en un campo de Coulomb incluyendo los efectos del espín. Esto mostrará algunas propiedades adicionales de la 
proyección del del impulso angular en un espacio de dimensión finita.

Para calcular la estructura fina de los niveles de energía del átomo de Hidrógeno, tomemos la reducción no relativista del Hamiltoniano

$$
H=H^{(0)}+\alpha^{2}\left[\frac{1}{2 r^{3}} \vec{L} \cdot \vec{S}-\frac{1}{2}\left(H^{(0)}+1 / r\right)^{2}+h_{D}\right]
$$

donde $\alpha=e^{2} /(\hbar c)$ es la constante de estructura fina. El Hamiltoniano no perturbado $H^{(0)}$ es el dado en (4.1) y $h_{D}$ es la interacción de Darwin.

El procedimiento estándar calcula las correcciones a los niveles de energía encontrando elementos matriciales del Hamiltoniano perturbado en la base de los operadores $L^{2}, S^{2}, J^{2}$ y $J_{z}$ que conmutan simultaneamente. Nosotros lo haremos por medio de una proyección de (4.22) en un espacio polinomial y de la diagonalización del Hamiltoniano finito obtenido mediante esta manera.

El primer punto a tratar es la forma explícita de la interacción de Darwin que debemos proyectar. Usualmente, el término no hermitiano y singular

$$
h_{D}=-\frac{1}{4 r^{2}} \frac{\partial}{\partial r}
$$

se convierte a una forma hermitiana (una delta de Dirac) a través de una integración por partes. Para estados con $l \neq 0$ este término puede removerse de (4.22), pero lo mantendremos para dar una descripción completa del Hamiltoniano perturbado. Sin embargo, debido a la dificultad numérica de tratar a una delta de Dirac y teniendo en cuenta la evidencia numérica de que la representación finita de (4.23) es una matriz con eigenvalores reales (cuando los nodos satisfacen (4.12), mantendremos la forma (4.23) en (4.22) en lugar de la forma usual $-(1 / 8) \nabla^{2}(1 / r)$.

Por otra parte, es necesario notar que la adición de la interacción spín óbita introduce una singularidad irregular en $r=0$, haciendo necesario buscar un cambio de variable que incorpore este comportamiento en la solución además de (4.3). Sin embargo, debido a la pequeñez de $\alpha^{2}$, podemos mantener (4.3), y por lo tanto los ceros de $L_{N}^{(1)}(2 \sqrt{2 \varepsilon r})$, como el conjunto de nodos radiales a pesar de que esta selección pueda dar lugar a una convergencia lenta, ya que no toma en cuenta propiamente la singularidad irregular en el origen.

De esta forma, el cambio de variable (4.2) produce un nuevo operador diferencial cuya forma matricial es

$$
\mathbf{H}^{\prime}=\mathbf{H}_{0}+\frac{\alpha^{2}}{2}\left[\mathbf{R}^{-3} \overrightarrow{\mathbf{L}} \cdot \vec{S}-\left(\mathbf{H}_{0}+\mathbf{R}^{-1}\right)^{2}-\frac{1}{2} \mathbf{R}^{-2}\left(\mathbf{D}_{r}-\sqrt{2 \varepsilon} \mathbf{1}\right)\right]
$$

donde $\mathbf{H}_{0}=I_{2} \otimes \mathbf{H}, \mathbf{H}$ dado por (4.6), $\mathbf{R}=I_{2 N M} \otimes R, \mathbf{D}_{r}=I_{2 N M} \otimes D_{r}, \mathbf{1}$ es la matriz identidad de dimensión $2 \tilde{N}$, y $\vec{L} \cdot \vec{S}$ está dado por

$$
2 \overrightarrow{\mathbf{L}} \cdot \vec{S}=\sigma_{x} \otimes \mathbf{L}_{\mathbf{x}}+\sigma_{y} \otimes \mathbf{L}_{y}+\sigma_{z} \otimes \mathbf{L}_{z} .
$$

El último término de (4.24), es la forma que el término de Darwin toma después del cambio de variable (4.2). Por razones de tipo numérico y para identificar la dependencia 
de los eigenvalores sobre los números cuánticos $n, l$ y $j$, es más conveniente diagonalizar la versión reducida en una dimensión de (4.24)

$$
H^{\prime}=H_{0}+\frac{\alpha^{2}}{2}\left[\lambda_{S L} R^{-3}-\left(H_{0}+R^{-1}\right)^{2}-\frac{1}{2} R^{-2}\left(D_{r}-\sqrt{2 \varepsilon} I_{N}\right)\right]
$$

donde $H_{0}=H_{r}+l(l+1) R^{-2} / 2, H_{r}$ dado por (4.10) y $\lambda_{S L}$ toma el valor $l / 2$ si $j=l+1 / 2$ $o-(l+1) / 2 j==l-1 / 2$, respectivamente. En la Tabla IV se muestra la estrucutra fina obtenida mediante la diagonalización de (4.26) para los primeros tres valores de $n$. Ya que la singularidad irregular del Hamiltoniano no se ha tomado en cuenta propiamente y el término de Darwin conduce a dificultades numéricas, es necesario tomar valores grandes de $N$ para alcanzar convergencia a los estados $s$, tal como se muestra. Nótese que para $l \neq 0$, la convergencia a los valores dados por la teoría de perturbaciones se alcanza para $N$ pequeña.

TABLA IV. Estructura fina del átomo de Hidrógeno. Las energías de amarre producidas por el teoría de perturbaciones $-E_{n l j}$ se comparan con las obtenidas por el presente método $-E_{n l j}^{N}$, para $N=50,100,800$.

\begin{tabular}{ccccc}
\hline State & $-E_{n l j}$ & $-E_{n l j}^{50}$ & $-E_{n l j}^{100}$ & $-E_{n l j}^{800}$ \\
\hline $1 s_{1 / 2}$ & 0.50000665991795 & 0.50000613488955 & 0.50000639259060 & 0.50000662026219 \\
& & & & \\
$2 s_{1 / 2}$ & 0.12500208122436 & 0.12500195038954 & 0.12500201493321 & 0.12500207221205 \\
$2 p_{1 / 2}$ & 0.12500208122436 & 0.12500208132882 & 0.12500208132927 & 0.12500208132965 \\
$2 p_{3 / 2}$ & 0.12500041624487 & 0.12500041624486 & 0.12500041624487 & 0.12500041624488 \\
& & & & \\
$3 s_{1 / 2}$ & 0.05555629554644 & 0.05555623744838 & 0.05555626615153 & 0.05555629166215 \\
$3 p_{1 / 2}$ & 0.05555629554644 & 0.05555629557858 & 0.05555629557882 & 0.05555629557903 \\
$3 p_{3 / 2}$ & 0.05555580221918 & 0.05555580221948 & 0.05555580221949 & 0.05555580221949 \\
$3 d_{3 / 2}$ & 0.05555580221918 & 0.05555580222221 & 0.05555580222221 & 0.05555580222221 \\
$3 d_{5 / 2}$ & 0.05555563777676 & 0.05555563777706 & 0.055555637777706 & 0.05555563777706 \\
\hline
\end{tabular}

\subsection{El átomo de Hidrógeno en un campo magnético}

Consideremos ahora el caso de un electrón ligado por un núcleo atómico y un campo magnético de intensidad $B$ apuntando en la dirección $z$. Emplearemos las mismas unidades que en las secciones anteriores $\mathrm{y}$, como unidad de la intensidad magnética, usaremos $B_{0}=m_{e}^{2} e^{3} c / \hbar^{3}=2.35 \times 10^{9} \mathrm{G}\left(m_{e}\right.$ y e son la masa y carga del electrón respectivamente), de tal forma que $B=\beta B_{0}$, donde $\beta$ es el campo magnético adimensional. El problema se desarrollará en coordenadas esféricas con la norma donde el potencial vectorial tiene 
componentes $A_{r}=A_{\theta}=0, A_{\varphi}=(B r \sin \theta) / 2$. Así, la ecuación de Schrödinger toma la forma

$$
\left[-\nabla^{2}-i \beta \frac{\partial}{\partial \varphi}+\frac{1}{4} \beta^{2} r^{2} \sin ^{2} \theta-\frac{2}{r}-\beta\right] \Psi=2 E \Psi
$$

El espín del electrón se ha tomado antialineado con el campo magnético para calcular el estado base, que es nuestro principal interés.

De acuerdo a nuestro método, necesitamos conocer la forma límite $\Psi_{\infty}(r, \theta, \varphi)$ de $\Psi$ para los estados ligados [cf. (4.2)]. Escribiendo $-E$ como $\varepsilon$, y tomando la forma límite de esta ecuación cuando $r \rightarrow \infty$ (suponiendo que las derivadas angulares de la función de onda están bien definidas para todos los valores de los ángulos) se obtiene

$$
\left[\frac{\partial^{2}}{\partial r^{2}}+i \beta \frac{\partial}{\partial \varphi}-\frac{1}{4} \beta^{2} r^{2} \sin ^{2} \theta+\beta\right] \Psi_{\infty}=2 \varepsilon \Psi_{\infty}
$$

La dependencia de esta ecuación en $\varphi$ puede factorizarse para producir

$$
\Psi_{\infty}(r, \theta, \varphi)=\Gamma(3 / 4+a) U(a, x) e^{i m \varphi}
$$

donde

$$
a=\frac{2 \varepsilon-\beta(1-m)}{\beta \sin \theta}, \quad x=\sqrt{\beta \sin \theta} r
$$

son los argumentos de la función parabólica de Weber $U(a, x)$ (ver [1]). La función Gamma que aparece en (4.29) es un factor de normalización que hace a $\Psi_{\infty}$ independiente de $\theta$ en $r=0$.

Ya que nuestro interés es calcular las energías del estado base, tomemos $m=0$ y substituyamos $\Psi=\Psi_{\infty} f$ en (4.27) para producir

$$
\begin{aligned}
& \left(f_{r r}+2 \frac{\gamma_{r}}{\gamma} f_{r}\right)+\frac{1}{r^{2}}\left(f_{\theta \theta}+2 \frac{\gamma_{\theta}}{\gamma} f_{\theta}\right) \\
& +\left[\frac{1}{r^{2}}\left(\frac{u_{\theta \theta}}{u}+\cot \theta \frac{u_{\theta}}{u}\right)+\frac{2}{r}\left(\frac{u_{r}}{u}+1\right)+2 \varepsilon\right] f \\
& +\frac{1}{r^{2} \sin ^{2} \theta} f_{\varphi \varphi}+i \beta f_{\varphi}=-2 E f
\end{aligned}
$$

en donde hemos definido

$$
\gamma(r, \theta)=r \sqrt{\sin \theta} u(r, \theta)
$$

y $u(r, \theta)=\Gamma(3 / 4+a) U(a, x), a$ y $x$ dados en $(4.30)$ con $m=0$.

Supongamos ahora que $f(r, \theta, \varphi)$ puede ser aproximada por una función de la forma $g(r, \theta) h(\varphi)$, donde $h$ es un polinomio trigonométrico en $\varphi$ y $g$ es un polinomio en dos variables (de hecho, $f$ tiene esa forma cuando $\beta=0$ ). Esta suposición permite proyectar la parte de (4.31) correspondiente a la coordenada $\varphi$ de acuerdo a las líneas ya discutidas. Además, puesto que $g(r, \theta)$ genera $N$ diferentes polinomios trigonométricos en $\theta$ cuando $r=r_{j}, j=1,2, \cdots, N$, y $K$ diferentes polinomios en $r$ cuando $\theta=\theta_{k}, k=1,2, \cdots, K, \mathrm{y}$ la representación de una derivada parcial está dada por bloques (recuérdese la definición 
de un producto de Kroenecker), podemos pedir que el $k$-ésimo bloque de la matriz que ahora represente $\partial / \partial r$ (en dos variables) se construya con la $k$-ésima línea de nodos $r_{j}^{k}, j=1,2, \cdots, N$ de acuerdo a la fórmula genérica (2.5) y similarmente, que el $j-$ ésimo bloque de la matriz que represente a $\partial / \partial \theta$ se construya con la línea de nodos $\theta_{k}^{j}$, $k=1,2, \cdots, K$ de acuerdo a la fórmula genérica (3.22). Debe tenerse presente que existe un orden en la construcción de tales matrices (recuérdese sus definiciones en términos de productos de Kroenecker) y que el índice que corre más rápidamente es el que corresponde a la variable $r$. Esto produce que la representación de $\partial / \partial r$ sea una matriz diagonal por bloques, mientras que la representación de $\partial / \partial \theta$ resulta ser una matriz dispersa (sparse matrix). Denotemos a estas matrices $K N \times K N$ por $D_{r}^{(2)}$ y $D_{\theta}^{(2)}$, respectivamente. Por otra parte, la representación de $\partial / \partial \varphi$ no sufre cambio en su definición (también es una matriz dispersa). Así tenemos finalmente que las nuevas representaciones de las derivadas parciales son

$$
\mathbf{D}_{r}=I_{M} \otimes D_{r}^{(2)}, \quad \mathbf{D}_{\theta}=I_{M} \otimes D_{\theta}^{(2)}, \quad \mathbf{D}_{\varphi}=\tilde{D_{\varphi}} \otimes I_{K N}
$$

La forma matricial que adopta una función coeficiente de (4.31) resulta ser el producto tensorial de $I_{M}$ con la matriz diagonal $K N \times K N$ cuyos elementos no nulos están dados por la función dada evaluada en los nodos $\left(r_{j}^{k}, \theta_{k}^{j}\right), j=1,2, \cdots, N, k=1,2, \cdots, K$, donde $j$ corre más rápidamente que $k$.

Así, la forma discreta de (4.31) es

$$
\begin{aligned}
& {\left[\left(\mathbf{D}_{r}^{2}+2 \mathbf{g}_{\mathbf{r}} \mathbf{D}_{r}\right)+\mathbf{r}^{-2}\left(\mathbf{D}_{\theta}^{2}+2 \mathbf{g}_{\theta} \mathbf{D}_{\theta}\right)\right.} \\
& +\mathbf{r}^{-2}\left(\mathbf{u}_{\theta \theta}+\cot \Theta \mathbf{u}_{\theta}\right)+2 \mathbf{r}^{-1}\left(\mathbf{u}_{\mathbf{r}}+\mathbf{1}\right)+2 \varepsilon \mathbf{1} \\
& \left.+\mathbf{c D}_{\varphi}^{2}+i \beta \mathbf{D}_{\varphi}\right] \mathbf{F}^{*}=-2 E^{*} \mathbf{F}^{*}
\end{aligned}
$$

donde $\varepsilon$ es tomado como un parámetro de prueba, $E^{*}$ y $\mathbf{F}^{*}$ son los aproximantes a la energía $E$ y eigenfunciones $\Psi$ (dividida por $\Psi_{\infty}$ y evaluada en los nodos), $\mathbf{D}_{r}, \mathbf{D}_{\theta}$ y $\mathbf{D}_{\varphi}$ están dadas en (4.33), $\mathbf{1}$ es la $M K N \times M K N$ matriz identidad y $\mathbf{g}_{\mathbf{r}}, \mathbf{r}, \mathbf{g}_{\theta}, \mathbf{u}_{\theta \theta}, \cot \Theta$, $\mathbf{u}_{\theta}, \mathbf{u}_{\mathbf{r}}, \mathrm{y} \mathbf{c}$, son las matrices diagonales correspondientes a las funciones [cf. (4.32)] $\gamma_{r} / \gamma$, $r, \gamma_{\theta} / \gamma, u_{\theta \theta} / u, \cot \theta, u_{\theta} / u, u_{r} / u$, y $1 /\left(r^{2} \sin ^{2} \theta\right)$, respectivamente.

Los nodos en la coordenada $\varphi$ pueden escogerse como los puntos equidistantes

$$
\varphi_{l}=-\pi+\frac{2 \pi l}{M}, \quad l=1,2, \cdots, M,
$$

tal como se ha hecho anteriormente, así que sólo queda por decir cómo escoger el conjunto de nodos $\left(r_{j}^{k}, \theta_{k}^{j}\right)$. Para esto, podemos seguir las ideas dadas en la sección 4.2 para lograr que la proyección de la ecuación radial se convierta en una matriz hermitiana [cf. (4.16)]. Denotemos por $\mathrm{d}_{\mathbf{r}} \mathrm{y} \mathrm{d}_{\theta}$ las entradas diagonales de $\mathrm{D}_{r}, \mathrm{y} \mathrm{D}_{\theta}$, respectivamente. Si imponemos la condición

$$
\mathrm{d}_{\mathbf{r}}=-\mathbf{g}_{\mathbf{r}}, \quad \mathrm{d}_{\theta}=-\mathbf{g}_{\theta}
$$

entonces $-\mathrm{D}_{r}^{2}-2 \mathbf{g}_{\mathbf{r}} \mathrm{D}_{r} \mathrm{y}-\mathrm{D}_{\theta}^{2}-2 \mathbf{g}_{\theta} \mathrm{D}_{\theta}$ son matrices positivas semidefinidas (salvo por una transformación de similitud) y por lo tanto, los eigenvalores de (4.34) son reales. Escrita 
en detalle, (4.36) resulta ser el sistema de $2 K N$ ecuaciones no lineales

$$
\begin{aligned}
\sum_{l=1}^{N} \frac{1}{\left(r_{j}^{k}-r_{l}^{k}\right)}+\frac{\gamma_{r}\left(r_{j}^{k}, \theta_{k}^{j}\right)}{\gamma\left(r_{j}^{k}, \theta_{k}^{j}\right)}=0, \\
\sum_{l=1}^{K} \frac{1}{2} \cot \frac{\left(\theta_{k}^{j}-\theta_{l}^{j}\right)}{2}+\frac{\gamma_{\theta}\left(r_{j}^{k}, \theta_{k}^{j}\right)}{\gamma\left(r_{j}^{k}, \theta_{k}^{j}\right)}=0,
\end{aligned}
$$

donde $j=1,2, \cdots, N, k=1,2, \cdots, K$ y $\gamma(r, \theta)$ está dada por (4.32). Así, si $\beta$ y $\varepsilon$ están dados numericamente, el conjunto de nodos $\left(r_{j}^{k}, \theta_{k}^{j}\right)$ puede encontrarse resolviendo el sistema (4.37).

En la Figura 1 se muestran los nodos obtenidos resolviendo (4.37) con $N=K=8$ para los casos (a) $\beta=0$, (b) $\beta=0.5$ y (c) $\beta=1$. Cuando $\beta=0$, el problema es separable en las coordenadas y tenemos el caso ya resuelto del átomo de Hidrógeno (sin campo magnético). Los nodos reflejan este hecho en su simetría angular. A medida que $\beta$ crece, las líneas radiales que unen a los puntos con un mismo valor de $\theta$ en la Figura 1.(a), decrecen en longitud y sus extremos se doblan hacia valores grandes de $|z|$. Los nodos reflejan también en estos casos la composición del potencial en una parte radial y otra dependiente de $z$.

Una vez determinadas las ecuaciones para los nodos, podemos proceder a resolver el eigenproblema de (4.34). Para obtener un eigenvalor de (4.34) para un valor $\beta^{\prime}$ del parámetro $\beta$, seguiremos un procedimiento recursivo en los parámetros $\beta$ y $\varepsilon$ que consiste en los siguientes pasos. Comencemos con los nodos que producen resultados exactos para $\beta=0$ y $\varepsilon=1 / 2$. Estos nodos son usados como puntos iniciales para resolver (4.37) $\operatorname{con} \beta$ igual a un pequeño incremento. Los nuevos nodos son usados para construir (4.34). Al resolver este problema de eigenvalores con rutinas estándares, se obtiene un valor para la energía del estado base, la cual se usa como un valor de $\varepsilon$ en la siguiente ronda de cálculos. Incrementamos de nuevo $\beta$ y usamos el último conjunto de nodos como puntos iniciales para encontrar un nuevo conjunto de puntos a través de (4.37) con los valores actuales de $\beta$ y $\varepsilon$. Resolviendo nuevamente (4.34), encontramos un nuevo $\varepsilon$ y así sucesivamente. El procedimiento se detiene cuando $\beta=\beta^{\prime}$, produciendo un eigenvalor para el estado base. Ahora cambiamos el incremento de $\beta$ y el procedimiento se reinicia. Después de varios incrementos iniciales de $\beta$, se buscan los dígitos estables en los eigenvalores obtenidos mediante esta forma. Para incrementar un poco la precisión de los resultados (y poder admitir números pares $K$ ), podemos usar la representación matricial de $d / d \theta$

$$
\begin{gathered}
D_{\theta}^{*}=T^{*} \bar{D}\left(T^{*}\right)^{-1}, \\
\bar{D}_{i j}=\left\{\begin{array}{ll}
\sum_{l=1}^{K} \cot \left(\theta_{i}-\theta_{l}\right), & i=j, \\
\cot \left(\theta_{i}-\theta_{j}\right), & i \neq j,
\end{array} \quad S_{i j}=\delta_{i j} \prod_{l \neq j}^{K} \sin \left(\theta_{j}-\theta_{l}\right),\right.
\end{gathered}
$$


dada en [11] (cf. sección 3.3). En tal caso, la matriz $D_{\theta}^{(2)}$ cambiará en forma correspondiente [y así lo hará también (4.33)] y el sumando de la segunda ecuación de (4.37) cambiará a $\cot \left(\theta_{k}^{j}-\theta_{l}^{j}\right)$. Esto es lo que hemos hecho al calcular las energías del estado base mostradas en la Tabla $\mathrm{V}$ para $N=4,6,8, K=N$, y $M=3$. Allí se comparan con los valores dados en [37].
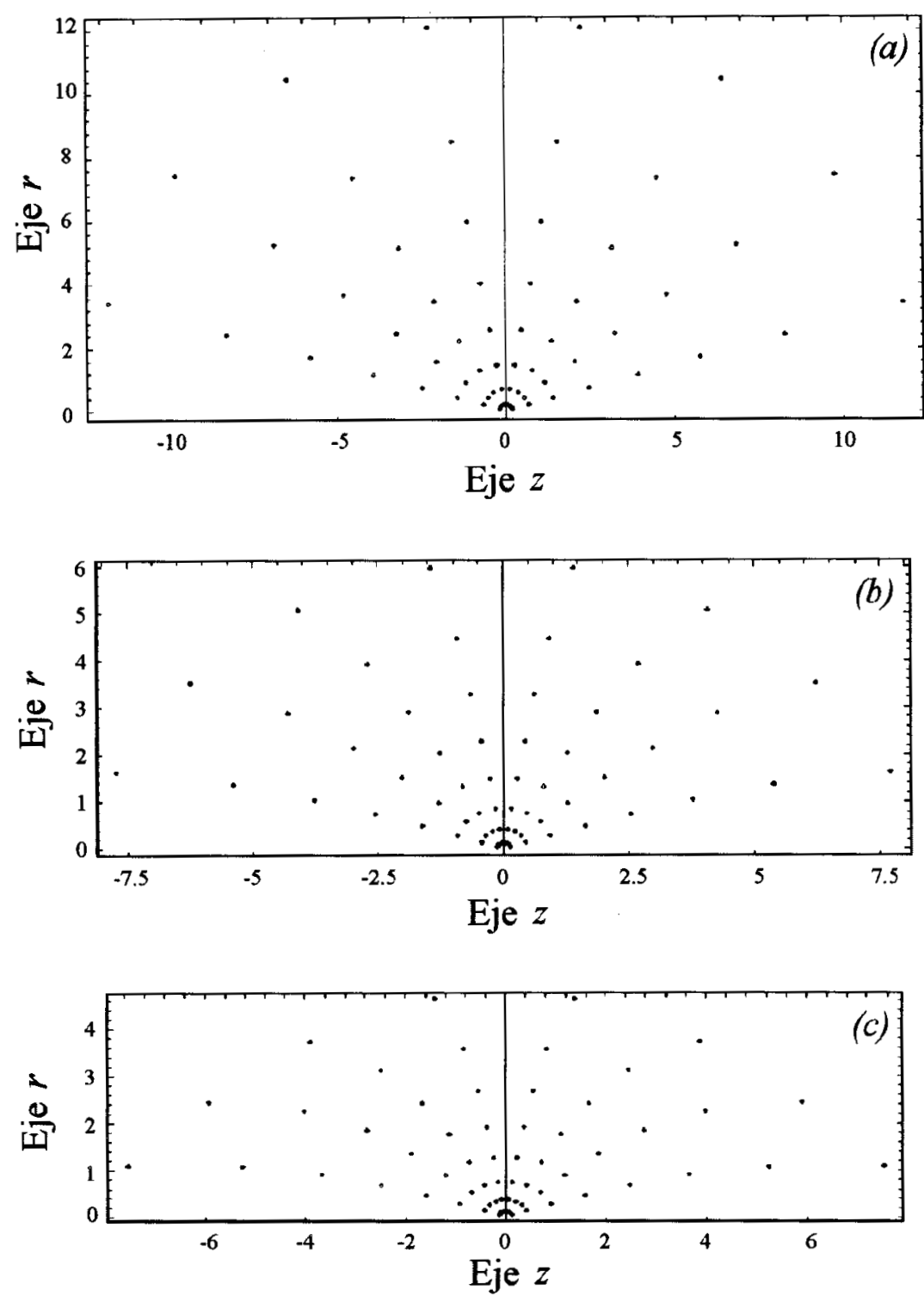

FIGURA 1. Ejemplos de nodos en $r$ y $\theta(N=K=8)$ para la discretización de la ecuación de onda del átomo de Hidrógeno en un campo magnético uniforme para tres valores de la intensidad magnética: $(a) \beta=0,(b) \beta=1 / 2,(c) \beta=1$. 
TABLA V. Energías de amarre $-E_{0}(M, K, N)$ del estado base de un tomo de Hidrógeno en un campo magnético uniforme calculadas de acuerdo al presente método. Sólo se dan los primeros dígitos estables y se comparan con las energías $-E_{0}$ dadas en [37].

\begin{tabular}{llllc}
\hline \multicolumn{1}{c}{$\beta$} & $-E_{0}(3,4,4)$ & $-E_{0}(3,6,6)$ & $-E_{0}(3,8,8)$ & $-E_{0}$ \\
\hline 0.0001 & 0.500049997500 & 0.500049997500 & 0.500049997500 & 0.500049997500 \\
0.001 & 0.50049975000 & 0.500499750000 & 0.500499750000 & 0.500499750000 \\
0.01 & 0.504961 & 0.504975 & 0.504975 & 0.504975002759 \\
0.1 & 0.547518 & 0.547527 & 0.547525 & 0.547526480401 \\
0.4 & 0.6647 & 0.6647 & 0.6646 & 0.664605379868 \\
0.6 & 0.727 & 0.7277 & 0.7273 & 0.727462287757 \\
0.8 & 0.78 & 0.782 & 0.782 & 0.782283393769 \\
1.0 & 0.83 & 0.83 & 0.83 & 0.831168896733 \\
\hline
\end{tabular}

Como puede verse, el método produce eigenvalores precisos y sus correspondientes eigenfunciones (obtenidas de los eigenvectores por medio de una interpolación) para valores no muy intensos del campo magnético $\left(\beta \approx 1\right.$, o bien, $\left.B \approx 10^{9} \mathrm{G}\right)$. El hecho de que esta técnica produzca resultados no aceptables cuando el campo es intenso $(\beta \gg 1)$ se debe a que (4.29) se vuelve una función oscilatoria cuando $a<0$ [o $\beta>2 \varepsilon$, cf. Eq. (4.30)] haciendo que la solución de (4.37) no pueda ser encontrada con alta precisión para $\beta \approx 1$. Para $\beta \gg 1$, la solución de (4.37) no es única, por lo que debe buscarse otra función $\Psi_{\infty}$ para este rango del parámetro. Sin embargo, nuestro interés aquí sólo ha sido mostrar un caso de importancia física donde el método pudiera aplicarse para darle credibilidad como un método de discretización de operadores diferenciales y así respaldar su aplicación a las ecuaciones de onda relativistas. 


\section{Capítulo 5}

\section{Ecuaciones relativistas discretas}

\subsection{Preliminares}

En este capítulo dejaremos aparte los aspectos computacionales de esta técnica para retomar su carácter de método de discretización para la Mecánica Cuántica Relativista. En esta parte no consideraremos ningún potencial, sólo casos libres, pues nuestro interés es sentar las bases de esta técnica de proyección para las ecuaciones de onda básicas y dejar para trabajos futuros su aplicación a problemas de mayor interés físico en la Electrodinámica Cuántica o de la Gravedad Cuántica. Primeramente tomaremos la ecuación de Klein-Gordon en $3+1$ dimensiones para proyectarla en el espacio de funciones $U_{\tilde{N}}$ (ver la sección 2.2) y resolveremos la ecuación matricial resultante usando la transformada de Fourier discreta dada por (2.60). Debido a la dificultad del problema, sólo tomaremos $1+1$ dimensiones para mostrar que la solución así obtenida puede aproximarse a la solución general de esta ecuación dada en términos de funciones de Green en el caso de una variable espacial. Algunas de las propiedades de esta técnica de solución serán usadas en el caso de la ecuación de Dirac, que por lo tanto se tratará enseguida. También en este caso sólo tomaremos una variable espacial. Mostraremos que el "modelo de ajedrez"de Feynmann para el propagador de una partícula relativista unidimensional [25] puede reobtenerse mediante esta técnica, además de otro tipo de soluciones.

\subsection{La ecuación de Klein-Gordon}

La notación que seguiremos aquí es aquella que se establece al final de la sección 2.2. Las variables $x, y$ y $z$ serán denotadas por $x^{1}, x^{2}$ y $x^{3}$ y el tiempo $t$ por $x^{4}$. La signatura será entonces $(-,-,-,+)$ y los impulsos asociados se denotarán en la forma usual $p^{\mu}$, $\mu=1,2,3,4\left(p^{4}=E\right) .{ }^{1}$ Las constantes $c$ y $\hbar$ serán tomadas como 1 y el espacio de

\footnotetext{
${ }^{1}$ Esta notación se usa por conveniencia ya que como se recordará, en los productos tensoriales del esquema discreto, la variable que corre más rápidamente es $x^{1}$, después $x^{2}$, y así sucesivamente, dejando al tiempo como la variable que corre más lentamente, para que en caso de
} 
proyección como el espacio $U_{\tilde{N}}$ formado por los productos tensoriales de los subespacios de la forma (2.20) para cada variable. $\tilde{N}=\prod_{1}^{4}\left(N_{j}-1\right)$ donde $N_{j}$ es el número de nodos que tomaremos en la coordenada $x^{\mu}$ para la discretización (ver la sección 2.2).

Como se sabe, la ecuación de Klein-Gordon

$$
\left(-\frac{\partial^{2}}{\partial t^{2}}+\nabla^{2}\right) \Psi=m^{2} \Psi
$$

aparece al substituir en la ecuación de energía-impulso de una partícula libre relativista

$$
E^{2}-p^{2}=m^{2}
$$

las reglas cuánticas

$$
\vec{p} \rightarrow-i \nabla, \quad E \rightarrow i \frac{\partial}{\partial t}
$$

Ya que nuestro esquema discreto reproduce dichas reglas con matrices finitas [ver Ec. (2.61)], la discretización de (5.1) produce la ecuación matricial

$$
\left[-\left(\mathbf{D}_{4}\right)^{2}+\left(\mathbf{D}_{1}\right)^{2}+\left(\mathbf{D}_{2}\right)^{2}+\left(\mathbf{D}_{3}\right)^{2}\right] \mathbf{f}=m^{2} \mathbf{f},
$$

salvo una transformación de similitud dada por

$$
\mathbf{S}=S_{4} \otimes S_{3} \otimes S_{2} \otimes S_{1}
$$

[que es la generalización de (2.44)] y que se incluye en el vector $\mathbf{f}$ por simplicidad, pero que su aplicación produce sólo un cambio de signo en nodos adyacentes en la forma asintótica de $\mathbf{f}$ [ver por ejemplo (2.24)]. Por lo tanto, dicho factor será ignorado en los resultados asintóticos que mostraremos más adelante.

Una primera observación sobre (5.4), es que corresponde a una ecuación de eigenvalores (en el caso continuo la ecuación de Klein-Gordon también es una ecuación de eigenvalores que acepta eigensoluciones para $m$ continua) Ya que en nuestro caso tenemos un operador matricial finito del lado izquierdo de la ecuación, con un espectro discreto por su misma naturaleza matricial, tendremos que aceptar una discretización en la masa de la partícula que represente dicha ecuación. Como contraparte, recordemos que existen problemas relativistas continuos que aceptan un espectro discreto para la masa (véase por ejemplo [2], donde se resuelve la ecuación de Klein-Gordon con un potencial de tipo electrostático). Así que (5.4) puede escribirse como

$$
\left[-\left(\mathbf{D}_{4}\right)^{2}+\left(\mathbf{D}_{1}\right)^{2}+\left(\mathbf{D}_{2}\right)^{2}+\left(\mathbf{D}_{3}\right)^{2}\right] \mathbf{f}_{q}=m_{q}^{2} \mathbf{f}_{q}
$$

donde $q=1,2, \cdots, N^{*}$ y $N^{*}=N_{1} N_{2} N_{3} N_{4}$. Un poco más adelante se verá que $m_{q}^{2}$ se aproxima a cualquier número real (positivo o negativo) cuando $N^{*} \rightarrow \infty$.

En la manera usual de calcular las soluciones Lorentz invariantes de la ecuación de KleinGordon (5.1) se emplea la transformada de Fourier y la teoría de distribuciones. Ver por requerir una función de onda en un tiempo dado, puedan manejarse facilmente los productos tensoriales. 
ejemplo [5] y [32] donde se obtienen la función de Pauli-Jordan y el propagador retrasado. En nuestro caso discreto, podemos resolver (5.6) usando también la transformada de Fourier pero en su forma discreta

$$
\mathbf{F}=\left(F^{4}\right)^{*} \otimes F^{3} \otimes F^{2} \otimes F^{1} .
$$

[dada en (2.60)] ya que la matriz $\mathbf{F}$ diagonaliza simultaneamente a todas las derivadas $\mathbf{D}_{\mu}$. Aplicando $\mathbf{F}$ a (5.6) y tomando en cuenta (2.62), se obtiene

$$
\left[\left(\mathbf{P}^{4}\right)^{2}-\left(\mathbf{P}^{3}\right)^{2}-\left(\mathbf{P}^{2}\right)^{2}-\left(\mathbf{P}^{1}\right)^{2}\right] \mathbf{g}_{q}=m_{q}^{2} \mathbf{g}_{q},
$$

donde

$$
\mathbf{g}_{q}=\mathbf{F f}_{q}
$$

es la transformada de Fourier discreta de $\mathbf{f}_{q}$ y las matrices diagonales $\mathbf{P}^{\mu}$ son las representaciones discretas de los impulsos $p^{\mu}$. Esta ecuación puede escribirse en la forma compacta (índices repetidos se suman)

$$
\left[\mathbf{P}_{\mu} \mathbf{P}^{\mu}\right] \mathbf{g}_{q}=m_{q}^{2} \mathbf{g}_{q},
$$

donde $\mathbf{P}_{\mu}=g_{\mu \nu} \mathbf{P}^{\nu}$ y $g_{\mu \nu}$ es la métrica asociada a la signatura empleada. Nótese que esta ecuación [o (5.8)] es la forma discreta de la ecuación de energía-impulso (5.2). De aquí se sigue que los eigenvalores $m_{q}^{2}$ están dados por las entradas diagonales de $\mathbf{P}_{\mu} \mathbf{P}^{\mu}$ de acuerdo a

$$
m_{q}^{2}=\left(p_{k}^{4}\right)^{2}-\left(p_{l}^{3}\right)^{2}-\left(p_{m}^{2}\right)^{2}-\left(p_{n}^{1}\right)^{2} .
$$

Aquí, el subíndice $q$ está relacionado con $k, l, m$ y $n$ a través del orden

$$
q=n+(m-1) N_{1}+(l-1) N_{1} N_{2}+(k-1) N_{1} N_{2} N_{3}
$$

donde primeramente se hace $n=1,2 \cdots, N_{1}$, y después $m=1,2 \cdots, N_{2}, l=1,2 \cdots, N_{3}$, y finalmente $k=1,2 \cdots, N_{4}$, de manera que $q=1,2, \cdots, N^{*}, N^{*}=N_{1} N_{2} N_{3} N_{4}$, generando los $N^{*}$ eigenvalores de (5.6). Ya que $p_{j}^{\mu}$ son raíces de polinomios de Hermite y éstos se vuelven densos en los números reales cuando el grado del polinomio crece hacia infinito, $m_{q}^{2}$ se aproxima a cualquier valor real cuando $N^{*} \rightarrow \infty$. Esto implica que la ecuación de Klein-Gordon así discretizada contiene soluciones de tipo taquiónico además de las soluciones con energía positiva o negativa. Los eigenvalores negativos son siempre mayores (o cuando menos iguales) en número que los positivos, dependiendo del número de variables espaciales que se tomen en cuenta. Por otra parte, puesto que las raíces de estos polinomios se encuentran simetricamente dispuestas alrededor de cero, el espectro de (5.6) es degenerado y esta degeneración depende de la naturaleza par o impar de cada $N_{\mu}$ y del número de variables consideradas, pues (5.11) produce distintas degeneraciones si tenemos por ejemplo, $1+1$ o $1+3$ variables. Este punto resulta importante al considerar la forma asintótica de la solución como se verá más adelante.

Para que (5.11) sea un eigenvalor de (5.8) se requiere que el eigenvector correspondiente $\mathbf{g}_{q}$ esté compuesto por ceros dondequiera excepto en el $q$-ésimo lugar donde deberá tener una 
componente distinta de cero que puede ser tomada igual a $1 .^{2}$ Debido a la degeneración existente, existen muchos valores de los índices $k, l, m$ y $n$ (y por lo tanto de $q$ ) para los cuales se obtiene el mismo eigenvalor $m_{q}^{2}$, por lo que la solución de (5.8) es

$$
\mathbf{g}_{m_{q}^{2}}=\sum_{\mathbf{P}_{\mu} \mathbf{P}^{\mu}=m_{q}^{2}} A_{q} \mathbf{g}_{q}
$$

donde $A_{q}$ es una constante y la suma se realiza sobre todo $q$ o sobre todos los $k, l, m$ y $n$ que hacen valer (5.11). Podemos obtener la solución de la ecuación de Klein-Gordon discreta (5.4) para un valor dado del eigenvalor $m_{q}^{2}$ antitransformando (5.13), es decir, aplicándole la matriz $\mathbf{F}^{\dagger}$ (recuérdese que $\mathbf{F}$ es unitaria), para obtener

$$
\mathbf{f}_{m_{q}^{2}}=\sum_{\mathbf{P}_{\mu} \mathbf{P}^{\mu}=m_{q}^{2}} A_{q} \mathbf{F}^{\dagger} \mathbf{g}_{q}
$$

Ya que $\mathbf{g}_{q}=\delta_{q s}$, el producto $\mathbf{F}^{\dagger} \mathbf{g}_{q}$ da como resultado la $q$-ésima columna de la matriz $\mathbf{F}^{\dagger}$ que denotaremos por $\left[\mathbf{F}^{\dagger}\right]_{q}$. Entonces

$$
\mathbf{f}_{m_{q}^{2}}=\sum_{\mathbf{P}_{\mu} \mathbf{P}^{\mu}=m_{q}^{2}} A_{q}\left[\mathbf{F}^{\dagger}\right]_{q}
$$

Si usamos la definición de $\mathbf{F}$ y la generalización de (2.50) se puede obtener el primer acercamiento a la forma asintótica de (5.15) cuando $N_{\mu} \rightarrow \infty$. Primeramente obsérvese que cualquier subíndice $s, s=1,2, \cdots, N^{*}$, puede reescibirse como una tétrada $k l m n$ pues estos cuatro índices determinan a $s$ de acuerdo a (5.12). Así, la componente $s$-ésima de $\mathbf{f}_{m_{q}^{2}}$ con $q$ escrito como $k^{\prime} l^{\prime} m^{\prime} n^{\prime}$ toma la forma asintótica

$$
\begin{aligned}
\lim _{N_{\mu} \rightarrow \infty}\left(\mathbf{f}_{m_{q}^{2}}\right)_{s} & =\Psi^{*}\left(x_{l}^{1}, x_{k}^{2}, x_{j}^{3}, x_{n}^{4}\right) \\
& =\sum_{\mathbf{P}_{\mu} \mathbf{P}^{\mu}=m_{q}^{2}} c_{k^{\prime} l^{\prime} m^{\prime} n^{\prime}} C_{N_{1}} C_{N_{2}} C_{N_{3}} C_{N_{4}} e^{i p_{n^{\prime}}^{4} x_{n}^{4}} e^{-i p_{j^{\prime}}^{3} x_{j}^{3}} e^{-i p_{k^{\prime}}^{2} x_{k}^{2}} e^{-i p_{l^{\prime}}^{1} x_{l}^{1}} \\
& =\sum_{\mathbf{P}_{\mu} \mathbf{P}^{\mu}=m_{q}^{2}} A_{q} e^{i\left(p_{\mu}\right)_{q}\left(x^{\mu}\right)_{s}}
\end{aligned}
$$

donde hemos definido $\Psi^{*}\left(x_{j}^{\mu}\right)$ como esta forma límite para enfatizar el hecho de que esta expresión es una proyección de la solución continua. La constante $C_{N_{\mu}}$ está dada por la forma genérica (2.25). Como puede verse, esta solución discreta contiene la forma funcional apropiada para conectarla con soluciones continuas Lorentz invariantes. Sin embargo, hay algunos puntos que deben tratarse con cuidado. Para obtener esa forma funcional, el proceso límite se realiza en forma simultánea para los cuatro parámetros $N_{\mu}$ y la suma se hace sobre los índices correspondientes a los nodos definidos por la degeneración

\footnotetext{
${ }^{2}$ Este tipo de vector corresponde a la proyección de una distribución centrada en un punto en nuestro espacio finito. Esto era de esperarse ya que las soluciones de la ecuación de Klein-Gordon en el espacio de impulsos son justamente distribuciones (ver por ejemplo [32])
} 
existente, la cual es independiente de que $N_{\mu}$ crezca o disminuya, ya que la degeneración depende sólo del carácter par-impar de ese parámetro y de la simetría de los ceros de Hermite, haciendo que aunque $N_{\mu}$ tienda hacia infinito, la dimensión del eigenespacio degenerado se mantiene constante (cambiando sólo si $N_{\mu}$ es par o impar), lo que implica que aunque $N_{\mu} \rightarrow \infty$, en la suma (5.16) se debe tomar siempre un mismo número finito de sumandos. Si recordamos que en el caso continuo el número de componentes de Fourier es infinito, esto puede parecer una falla en la aproximación del método discreto. Sin embargo, esto no quiere decir que las soluciones discretas y continuas no puedan conectarse entre sí puesto que los ceros de Hermite se vuelven densos en los reales cuando $N_{\mu} \rightarrow \infty$, de manera que en este límite hay muchos puntos muy cercanos entre sí siendo posible aproximar la suma de (5.16) por una integral, tal como se verá enseguida.

Con el objeto de simplificar los resultados, consideraremos sólo $1+1$ variables $x^{1}=x \mathrm{y}$ $x^{4}=t$ con sus impulsos $p^{1}=p$ y $p^{4}=E$. Además, tomaremos el mismo número de nodos en las dos coordenadas, $N_{1}=N$ y $N_{4}=N$, de manera que la ecuación (5.11) toma la forma

$$
m_{q}^{2}=E_{n}^{2}-p_{l}^{2}, \quad q=l+(n-1) N
$$

con $l, n=1,2, \cdots, N$ (hemos cambiado la notación de los índices para no confundir con la masa). Nuevamente $m_{q}^{2}$ se aproxima a cualquier valor real cuando $N \rightarrow \infty$ y presenta degeneración. Ilustremos esto con $N$ par y para $m_{q}^{2} \geq 0$. En este caso el eigenvalor $m^{2}=0$ es $2 N$-degenerado mientras que los demás son sólo 4-degenerados. Por lo tanto, las sumas que componen la solución (5.15) de la ecuación de Klein-Gordon sólo contienen cuatro sumandos, excepto en el caso nulo donde hay $2 N$ de ellos, de tal forma que ajustándonos estrictamente a estos resultados, la forma límite de nuestra ecuación discreta (5.15) sólo consiste en una suma de 4 exponenciales. Sin embargo, debido al adensamiento de los ceros de Hermite en los reales, existe una infinidad de puntos suficientemente cercanos a las hipérbolas $E= \pm\left(m_{q}^{2}+p^{2}\right)^{1 / 2}$ en el plano $p-E$ haciendo posible substituir la degeneración discreta por todo el conjunto de puntos de esas curvas. Este hecho se muestra esquematicamente en la Figura 2.

De esta manera, en la medida que $N \rightarrow \infty$, podemos substituir la suma

$$
\mathbf{f}_{m_{q}^{2}}=\sum_{E_{n}^{2}-p_{l}^{2}=m_{q}^{2}} A_{q}[\mathbf{F}]_{q} .
$$

por una integral, pero antes es necesario examinar el comportamiento de las constantes $C_{N}$ 's que aparecen en (5.16). Nótese que el $j$-ésimo cero de $H_{N}(x)$ puede aproximarse por la fórmula de puntos equidistantes

$$
x_{j} \approx\left(\frac{2 j-N-1}{\sqrt{2 N}}\right) \frac{\pi}{2},
$$

así que de acuerdo a su definición (2.25), la constante $C_{N}$ resulta proporcional a la diferencia entre dos ceros adyacentes:

$$
C_{N}=\frac{1}{\sqrt{2 \pi}} \Delta x
$$




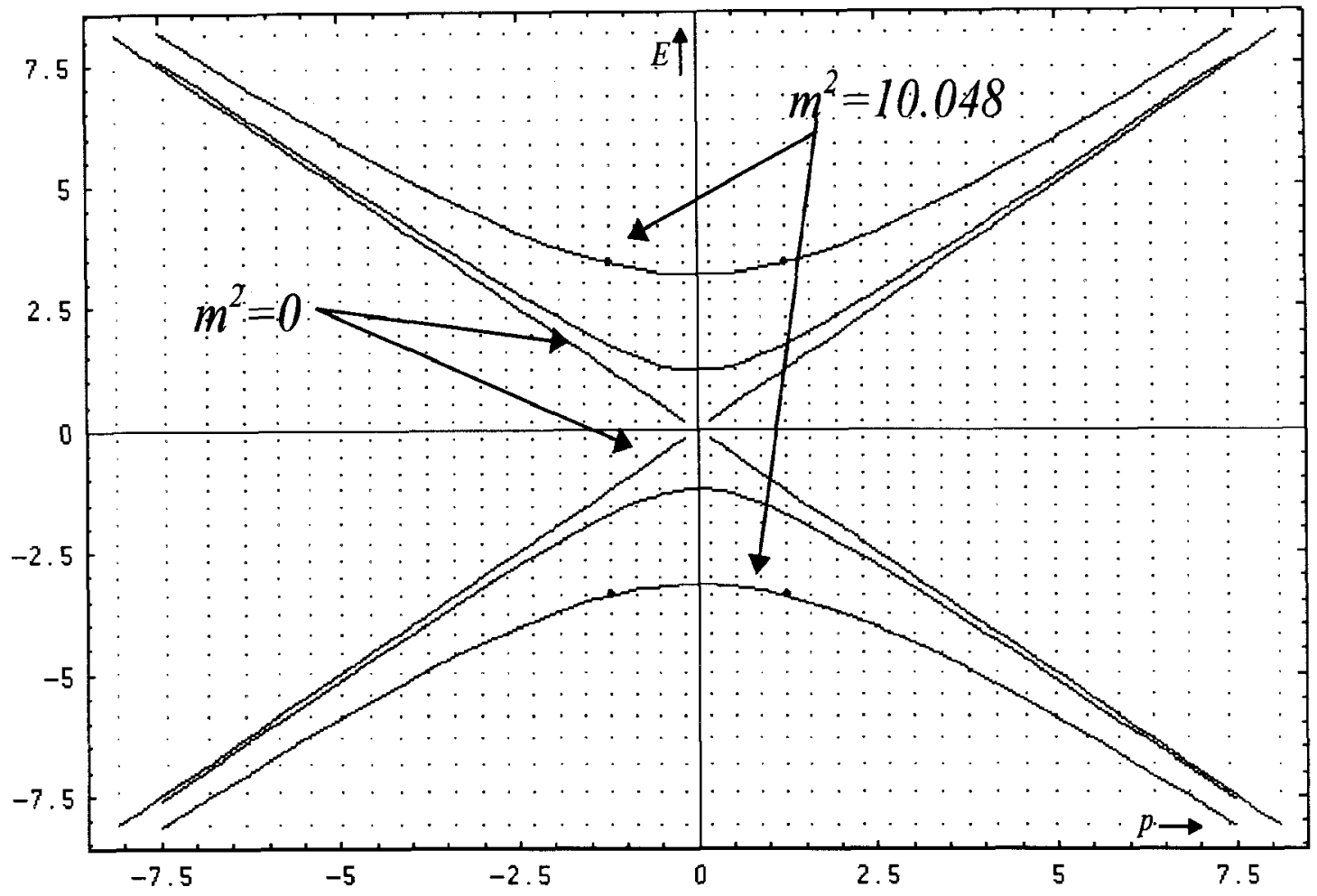

FIGURA 2. Puntos del espacio discreto de Energía-Impulso en $1+1$ variables con $N=$ $M=40$. Se muestran las hipérbolas que pasan por los puntos que hacen $m^{2}=10.048$ y $m^{2}=0$. En este caso el eigenvalor nulo es $2 N$-degenerado, mientras que los demás eigenvalores $\left(m^{2} \neq 0\right)$ son 4-degenerados.

donde $\Delta x=x_{j+1}-x_{j}$ es el paso o diferencia espacial. Ya que estamos usando el mismo número de nodos para $x$ y $t$, tanto el paso espacial como el temporal se escalan en la misma manera. Para aligerar la notación eliminaremos los subíndices $m_{q}^{2}, n$ y $l$ de $f_{m_{q}^{2}}$, $E_{n}^{2}$ y $p_{l}^{2}$, respectivamente. Así, la forma límite de $\mathbf{f}$ para energías positivas es

$$
\lim _{N \rightarrow \infty} \mathbf{f}=\Psi^{*}\left(x_{j}, t_{k}\right)=\frac{1}{2 \pi} \sum_{E=\left(m^{2}+p^{2}\right)^{1 / 2}} A(p) e^{i\left(-E t_{k}+p x_{j}\right)} \Delta E \Delta p
$$

Ahora estamos en condiciones de aproximar esta suma por la integral doble

$$
\begin{aligned}
\Psi\left(x_{j}, t_{k}\right) & =\frac{1}{2 \pi} \int_{-\infty}^{\infty} d E \int_{-\infty}^{\infty} d p \delta\left(E-\sqrt{m^{2}+p^{2}}\right) A(p) e^{i\left(-E t_{k}+p x_{j}\right)} \\
& =\frac{1}{2 \pi} \int_{-\infty}^{\infty} A(p) e^{i\left(-t_{k} \sqrt{m^{2}+p^{2}}+p x_{j}\right)} d p .
\end{aligned}
$$


La última integral puede calcularse en términos de las condiciones iniciales

$$
\xi(x)=\Psi(x, 0), \quad \eta(x)=\left.\frac{\partial \Psi}{\partial t}\right|_{t=0},
$$

si separamos la exponencial que depende de $\sqrt{m^{2}+p^{2}}$ y usamos las fórmulas para transformadas de Fourier y de Hankel dadas en [23] y algunos teoremas de convolución. De esta forma se obtiene

$$
\begin{aligned}
\Psi(x, t) & =\frac{1}{2} \xi(x+t)+\frac{1}{2} \xi(x-t)+\frac{1}{2} \int_{x-t}^{x+t} \eta(s) J_{0}\left(m \sqrt{t^{2}-(x-s)^{2}}\right) d s \\
& -\frac{m t}{2} \int_{x-t}^{x+t} \xi(s) \frac{J_{1}\left(m \sqrt{t^{2}-(x-s)^{2}}\right)}{\sqrt{t^{2}-(x-s)^{2}}} d s,
\end{aligned}
$$

donde hemos suprimido los índices de $x$ y $t$ para clarificar la notación. $J_{0}(x)$ y $J_{1}(x)$ son funciones de Bessel. Ésta es la bien conocida solución general de la ecuación de KleinGordon en $1+1$ coordenadas (ver por ejemplo [38]) escrita en términos de funciones de Green.

Esto muestra que nuestra técnica reproduce las soluciones de esta ecuación relativista con matrices de dimensión finita que preservan las relaciones cuánticas entre impulsos y derivadas, siempre que consideremos un subespacio de funciones degeneradas "cercano" pero mucho más grande que el subespacio producido por el mismo método.

\subsubsection{Ecuación de continuidad}

Una de las principales razones por la que la ecuación de Klein-Gordon para una partícula no es bien aceptada, es la dificultad de definir una densidad de probabilidad positiva definida. Por lo tanto resulta de interés examinar la forma que la ecuación de continuidad adopta en este esquema discreto. Para este fin seguiremos la forma estándar de derivación de dicha ecuación, que escrita en las unidades usadas en esta sección es

$$
\frac{\partial}{\partial t}\left(\Psi^{*} \frac{\partial}{\partial t} \Psi-\Psi \frac{\partial}{\partial t} \Psi^{*}\right)-\nabla \cdot\left(\Psi^{*} \nabla \Psi-\Psi \nabla \Psi^{*}\right)=0 .
$$

Como se sabe, esta ecuación se obtiene restando el producto de $\Psi$ por el conjugado del operador de Klein-Gordon del producto de $\Psi^{*}$ por dicho operador. Nuestro objetivo será obtener una ecuación matricial que sea en cierto modo el equivalente de (5.25) y averiguar si en nuestro esquema discreto tampoco es posible definir una probabilidad que sea positiva definida.

Por simplicidad, usaremos sólo $1+1$ coordenadas con $N_{1}$ nodos para la coordenada $x$ y $N_{2}$ para $t$. Obsérvese que por (5.15) una solución básica de la ecuación de Klein-Gordon discreta

$$
\left[-\left(\mathbf{D}_{t}\right)^{2}+\left(\mathbf{D}_{x}\right)^{2}\right] \mathbf{f}_{q}=m_{q}^{2} \mathbf{f}_{q}
$$


es justamente la columna $q$-ésima de $\mathbf{F}^{\dagger}$, es decir, las columnas de la matriz $\mathbf{F}^{\dagger}$ forman una base para cualquier solución de Klein-Gordon. Entonces basta considerar la transformación de (5.26) con una de esas columnas. Sin embargo, es conveniente reescribir (5.26) como la relación matricial

$$
\left[-\left(\mathbf{D}_{t}\right)^{2}+\left(\mathbf{D}_{x}\right)^{2}\right] \mathbf{F}^{\dagger}=\mathbf{F}^{\dagger} \mathbf{M}^{2}
$$

donde $\mathbf{M}^{2}$ es la matriz diagonal formada por los eigenvalores $m_{q}^{2}, q=1,2, \cdots, N_{1} N_{2}$, para usar las relaciones

$$
\mathbf{F D}_{t}=\mathbf{D}_{t} \mathbf{F}+i(\mathbf{F E}+\mathbf{E F}) \quad \mathbf{F}^{\dagger} \mathbf{D}_{t}=\mathbf{D}_{t} \mathbf{F}^{\dagger}-i\left(\mathbf{F}^{\dagger} \mathbf{E}+\mathbf{E} \mathbf{F}^{\dagger}\right)
$$

y

$$
\mathbf{F} \mathbf{D}_{x}=\mathbf{D}_{x} \mathbf{F}-i(\mathbf{F P}+\mathbf{P F}) \quad \mathbf{F}^{\dagger} \mathbf{D}_{x}=\mathbf{D}_{x} \mathbf{F}^{\dagger}+i\left(\mathbf{F}^{\dagger} \mathbf{P}+\mathbf{P F}^{\dagger}\right)
$$

que se pueden obtener fácilmente transponiendo y/o conjugando las ecuaciones (2.61) y usando el hecho de que $\mathbf{D}_{\mu}$ es antisimétrica y $\mathbf{F}$ es simétrica. Las matrices diagonales $\mathbf{E}$ y $\mathbf{P}$ de (5.28) y (5.29), han sido ya definidas en las ecuaciones (2.61). Restando el producto de $\mathbf{F}$ por (5.27) del producto de $\mathbf{F}^{\dagger}$ por la conjugada de (5.27), se obtiene

$$
\left[\mathbf{F}\left(\mathbf{D}_{t}\right)^{2} \mathbf{F}^{\dagger}-\mathbf{F}^{\dagger}\left(\mathbf{D}_{t}\right)^{2} \mathbf{F}\right]+\left[\mathbf{F}^{\dagger}\left(\mathbf{D}_{x}\right)^{2} \mathbf{F}-\mathbf{F}\left(\mathbf{D}_{x}\right)^{2} \mathbf{F}^{\dagger}\right]=0 .
$$

Mediante las relaciones (5.28) y (5.29), esta ecuación puede reescribirse como

$$
\mathbf{D}_{t}\left(\mathbf{F}^{\dagger} \mathbf{D}_{t} \mathbf{F}-\mathbf{F D}_{t} \mathbf{F}^{\dagger}\right)-\mathbf{D}_{x}\left(\mathbf{F}^{\dagger} \mathbf{D}_{x} \mathbf{F}-\mathbf{F D}_{x} \mathbf{F}^{\dagger}\right)+\mathbf{Q},
$$

donde $\mathbf{Q}=\mathbf{F E F}{ }^{\dagger} \mathbf{E}-\mathbf{F}^{\dagger} \mathbf{E F E}+\mathbf{F}^{\dagger} \mathbf{P F P}-\mathbf{F P F}^{\dagger} \mathbf{P}$.

Para hace contacto con la ecuación de continuidad, debemos observar que al reescribir la ecuación de Klein-Gordon discreta en la forma (5.27), hemos incluído todos los vectores de onda (en la matriz $\mathbf{F}^{\dagger}$ ) en lugar de tomar uno sólo de ellos (pues la probabilidad debe estar definida en términos de una función de onda y su conjugada). Por lo tanto, deberemos fijar en (5.30) una de las columnas de $\mathbf{F}^{\dagger}$, dando como consecuencia que sean las componentes diagonales de esa ecuación [o de (5.31)] las que buscamos. Analizando la forma de la matriz $\mathbf{Q}$, puede mostrarse que sus elementos diagonales son siempre cero, por lo que la relación que podríamos asociar en este esquema discreto con la ecuación de continuidad aplicada a, digamos, el vector $\mathbf{f}_{q}$, es el elemento diagonal $q q$ de

$$
\mathbf{D}_{t}\left(\mathbf{F}^{\dagger} \mathbf{D}_{t} \mathbf{F}-\mathbf{F D}_{t} \mathbf{F}^{\dagger}\right)-\mathbf{D}_{x}\left(\mathbf{F}^{\dagger} \mathbf{D}_{x} \mathbf{F}-\mathbf{F D}_{x} \mathbf{F}^{\dagger}\right)
$$

ecuación que muestra parecido (bajo las substituciones usuales hechas en este trabajo) con (5.25) para $1+1$ variables. El término

$$
\left(\Psi^{*} \frac{\partial}{\partial t} \Psi-\Psi \frac{\partial}{\partial t} \Psi^{*}\right)
$$

que usualmente se relaciona a la densidad de probabilidad, tiene su homónimo discreto dado por

$$
\mathbf{F}^{\dagger} \mathbf{D}_{t} \mathbf{F}-\mathbf{F D}_{t} \mathbf{F}^{\dagger}
$$

cuyos elementos diagonales no son positivos definidos. Así vemos que nuestra técnica produce una ecuación de Klein-Gordon discreta que hereda la imposibilidad de definir una densidad de probabilidad positiva. 


\subsubsection{Una raíz de la ecuación de Klein-Gordon discreta}

Otro aspecto importante que tratar antes de concluír esta sección (por la relación que pudiera tener con la ecuación de Dirac), es investigar la posibilidad de encontrar una raíz cuadrada de la ecuación de Klein-Gordon discreta que sea lineal en las derivadas.

Nuevamente tomaremos sólo $1+1$ coordenadas como en la subsección anterior y después generalizaremos a $1+3$.

La matriz $D^{0}$ definida a través de (2.5) usando ceros de Hermite y que representa la derivada unidimensional en el espacio de funciones (2.20), en cuyos términos se encuentran definidas las matrices de derivación parcial $\mathbf{D}_{\mu}$ a través de (2.54), posee una propiedad clave para el objetivo de esta subsección. Denotemos por $U$ la matriz $N \times N$ que tiene 1 's en su antidiagonal y ceros en sus otras entradas. Es decir, si $U_{j k}=\delta_{j, N-k+1}$, entonces se tiene que

$$
U^{-1} D^{0} U=U D^{0} U=-D^{0}
$$

En el caso de dos variables, cuando se han seleccionado $N_{1}$ nodos para $x$ y $N_{2}$ para $t$, defínase la matriz

$$
\mathbf{U}=1 \otimes U, \quad\left(\mathbf{U}^{-1}=\mathbf{U}\right)
$$

donde 1 es la matriz identidad de $N_{2} \times N_{2}$, mientras que ahora la dimensión de $U$ es $N_{1}$. Entonces tendremos las relaciones

$$
\mathbf{U D}_{t} \mathbf{U}=\mathbf{D}_{t}, \quad \mathbf{U D}_{x} \mathbf{U}=-\mathbf{D}_{x} .
$$

Usando estas ecuaciones no es difícil mostrar que una raíz lineal en las derivadas del operador discreto

$$
\mathbf{C}^{2}=\left(\mathbf{D}_{t}\right)^{2}-\left(\mathbf{D}_{x}\right)^{2}
$$

es la matriz

$$
\mathbf{C}=\mathbf{U D}_{t}-\mathbf{U D}_{x}
$$

En efecto, multiplicando (5.39) consigo mismo y tomando en cuenta (5.37) y que $\mathbf{D}_{t} \mathrm{y}$ $\mathbf{D}_{x}$ conmutan, se tiene

$$
\mathbf{U}\left(\mathbf{D}_{t}-\mathbf{D}_{x}\right) \mathbf{U}\left(\mathbf{D}_{t}-\mathbf{D}_{x}\right)=\left(\mathbf{U D}_{t} \mathbf{U}-\mathbf{U D}_{x} \mathbf{U}\right)\left(\mathbf{D}_{t}-\mathbf{D}_{x}\right)=\mathbf{C}^{2} .
$$

Otra raíz se obtiene cambiando el signo - en (5.39) por +. Por lo tanto, dos raíces de la ecuación de Klein-Gordon discreta en $1+1$ variables son

$$
\mathbf{U}\left(\mathbf{D}_{t}-\mathbf{D}_{x}\right) \mathbf{f}=i m \mathbf{f}, \quad \mathbf{U}\left(\mathbf{D}_{t}+\mathbf{D}_{x}\right) \mathbf{g}=i m \mathbf{g} .
$$

que pueden escribirse en la forma

$$
\left(\mathbf{D}_{t}-\mathbf{D}_{x}\right) \mathbf{f}=i m \mathbf{g}, \quad\left(\mathbf{D}_{t}+\mathbf{D}_{x}\right) \mathbf{g}=i m \mathbf{f} .
$$

donde hemos definido

$$
\mathbf{f}=\mathbf{U g}
$$


Estas dos raíces pueden interpretarse como las componentes de la ecuación de Dirac discreta en $1+1$ variables en cierta representación [ver (5.50)] en el caso particular en que (5.43) se mantenga, es decir, en en caso en que las componentes se encuentren desacopladas hasta la trasformación dada por la matriz $\mathbf{U}$.

Para extender el procedimiento de raíz cuadrada a $1+3$ variables, definamos las matrices

$$
\begin{aligned}
& \mathrm{U}^{4}=I_{4} \otimes U_{3} \otimes U_{2} \otimes U_{1} \\
& \mathrm{U}^{3}=I_{4} \otimes U_{3} \otimes U_{2} \otimes U_{1} \\
& \mathrm{U}^{2}=I_{4} \otimes I_{3} \otimes U_{2} \otimes U_{1} \\
& \mathrm{U}^{1}=I_{4} \otimes I_{3} \otimes I_{2} \otimes U_{1}
\end{aligned}
$$

(nótese que $\mathbf{U}_{4}=\mathbf{U}_{3}$ ), donde $I_{k}$ y $U_{k}$ son las matrices identidad y la de $1^{\prime} s$ en la antidiagonal, respectivamente, de dimensión $N_{k}$. Entonces, usando las propiedades del producto tensorial de matrices, las definiciones de las matrices $\mathbf{D}_{\mu}$ en términos de las matrices individuales $D_{\mu}^{0}$, la conmutatividad de las matrices $\mathbf{D}_{\mu}$ y la propiedad (5.35) que ahora se lee $U_{k} D_{k}^{0} U_{k}=-D_{k}^{0}, k=1,2,3$, se puede mostrar que la matriz

$$
\mathbf{C}=\mathbf{U}^{4} \mathbf{D}_{4}-\mathbf{U}^{3} \mathbf{D}_{3}-\mathrm{U}^{2} \mathbf{D}_{2}-\mathbf{U}^{1} \mathbf{D}_{1},
$$

es una raíz de la representación discreta del Dalambertiano

$$
\mathbf{C}^{2}=\mathbf{D}^{\mu} \mathbf{D}_{\mu}=\left(\mathbf{D}_{4}\right)^{2}-\left(\mathbf{D}_{3}\right)^{2}-\left(\mathbf{D}_{2}\right)^{2}-\left(\mathbf{D}_{1}\right)^{2} .
$$

Otras raíces de esta matriz se obtienen cambiando cualquiera de los signos en (5.45). Finalizaremos esta subsección generalizando la propiedad (5.35) a este caso. Un poco de álgebra muestra que ahora tendremos

$$
\left\{\mathbf{U}^{\mu} \mathbf{D}_{\mu}, \mathbf{U}^{\nu} \mathbf{D}_{\nu}\right\}=2 g_{\mu \nu}\left(\mathbf{D}_{\mu}\right)^{2},
$$

donde no hay suma entre índices repetidos y $\{$,$\} denota el anticonmutador entre matrices.$ En la siguiente sección tratamos la discretización de la ecuación de Dirac. Ya que las herramientas usadas han sido establecidas aquí, el tratamiento del caso de Dirac será dado en forma más concisa.

\subsection{La ecuación de Dirac}

Para establecer las bases de discretización en el caso de la ecuación de Dirac sólo usaremos $1+1$ variables y dejaremos como problema abierto la extensión de esta técnica a problemas de mayor interés físico.

Para aplicar nuestro método en este caso, requerimos de una representación específica de la ecuación de Dirac. En esta sección usaremos las dos representaciones dadas en [31] y denotadas por RI y RII. Así, si la función de onda está dada por

$$
\Psi=\left(\begin{array}{l}
\psi \\
\phi
\end{array}\right)
$$


la ecuación de Dirac (en las unidades usadas en la sección anterior) toma la forma

$$
\left(\begin{array}{c}
\frac{\partial \psi}{\partial t}-\frac{\partial \psi}{\partial x} \\
\frac{\partial \phi}{\partial t}+\frac{\partial \phi}{\partial x}
\end{array}\right)=m\left(\begin{array}{c}
-\phi \\
\psi
\end{array}\right),
$$

en la primera representación (RI), y

$$
\left(\begin{array}{l}
\frac{\partial \psi}{\partial t}-\frac{\partial \psi}{\partial x} \\
\frac{\partial \phi}{\partial t}+\frac{\partial \phi}{\partial x}
\end{array}\right)=-i m\left(\begin{array}{c}
\phi \\
\psi
\end{array}\right),
$$

en la segunda representación (RII), en la cual comenzamos a trabajar. De acuerdo al método, la forma discreta de (5.50) está dada por las ecuaciones

$$
\left(\mathbf{D}_{t}-\mathbf{D}_{x}\right) \mathbf{f}_{q}=-i m_{q} \mathbf{g}_{q}, \quad\left(\mathbf{D}_{t}+\mathbf{D}_{x}\right) \mathbf{g}_{q}=-i m_{q} \mathbf{f}_{q},
$$

que, como escritas como ecuaciones matriciales (lo cual resultará más conveniente), son

$$
\left(\mathbf{D}_{t}-\mathbf{D}_{x}\right) \mathbf{F}=-i \mathbf{G M}, \quad\left(\mathbf{D}_{t}+\mathbf{D}_{x}\right) \mathbf{G}=-i \mathbf{F M},
$$

donde $\mathbf{M}$ es una matriz diagonal con los valores $m_{q}$ de la masa discretizada a lo largo de su diagonal principal y $\mathbf{F}$ y $\mathrm{G}$ denotan las matrices cuyas columnas son $[\mathbf{f}]_{q}$, $[\mathbf{g}]_{q}$, $q=1,2, \cdots, N^{2}$.

Denotemos ahora por $C_{x}$ y $S_{x}$ la parte real e imaginaria de la transformada de Fourier discreta $F_{x}$ unidimensional dada en (2.21), i.e., $F_{x}=C_{x}+i S_{x}$. De (2.23) se ve que $D_{x}^{0}$ intercambia una parte en la otra de acuerdo a

$$
D_{x}^{0} C_{x}=-S_{x} P, \quad D_{x}^{0} S_{x}=C_{x} P .
$$

Por lo tanto, este hecho sugiere buscar una solución de (5.52) en la forma

$$
\mathbf{F}=\left(C_{t} \otimes C_{x}\right) \mathbf{A}_{1}-\left(S_{t} \otimes S_{x}\right) \mathbf{A}_{2}, \quad \mathbf{G}=-i\left[\left(S_{t} \otimes C_{x}\right) \mathbf{B}_{1}+\left(C_{t} \otimes S_{x}\right) \mathbf{B}_{2}\right],
$$

donde las A's y B's son matrices incógnitas a determinarse. Nótese que el significado de F ha cambiado. La substitución de (5.54) en (5.52), produce las condiciones

$$
\mathbf{A}_{1} \mathbf{E}-\mathbf{A}_{2} \mathbf{P}=\mathbf{B}_{1} \mathbf{M}, \quad \mathbf{A}_{2} \mathbf{E}-\mathbf{A}_{1} \mathbf{P}=\mathbf{B}_{2} \mathbf{M},
$$

y

$$
\mathbf{B}_{1} \mathbf{E}+\mathbf{B}_{2} \mathbf{P}=\mathbf{A}_{1} \mathbf{M}, \quad \mathbf{B}_{2} \mathbf{E}+\mathbf{B}_{1} \mathbf{P}=\mathbf{A}_{2} \mathbf{M},
$$

donde $\mathbf{E}$ y $\mathbf{P}$ son las matrices diagonales de energía y impulso usadas en la sección anterior. Para obtener (5.55) y (5.56) hemos supuesto que $\mathbf{A}_{j}$ y $\mathbf{B}_{j}$ son matrices diagonales y para asegurar la consistencia de (5.55) y (5.56), deberemos tener que

$$
\mathbf{E}^{2}-\mathbf{P}^{2}=\mathbf{M}^{2}
$$


(una relación ya encontrada antes), y $\mathbf{A}_{j}$ y $\mathbf{B}_{j}$ deben ser matrices no singulares. Para que esta última condición se satisfaga, se requiere que el número $N$ de nodos sea par, como se mostrará enseguida.

Una solución de (5.55) [o (5.56)] es

$$
\mathbf{A}_{1}=1, \quad \mathbf{A}_{2}=\mathbf{P E}^{-1}, \quad \mathbf{B}_{1}=\mathbf{M E}^{-1}, \quad \mathbf{B}_{2}=\mathbf{0},
$$

donde 0 and 1 son las $N^{2} \times N^{2}$ matrices nula e identidad respectivamente. Ya que las entradas diagonales de $\mathbf{E}$ y $\mathbf{P}$ son ceros de $H_{N}(x)$ y estos puntos se encuentran simétricamente localizados alrededor de cero, esas matrices son no singulares sólo cuando $N$ es par. Así, (5.54) resulta en

$$
\mathbf{F}=\left(C_{t} \otimes C_{x}\right)-\left(S_{t} \otimes S_{x}\right) \mathbf{P E}^{-1}, \quad \mathbf{G}=-i\left(S_{t} \otimes C_{x}\right) \mathbf{M E}^{-1} .
$$

Los razonamientos hechos en la sección anterior al considerar los valores de $E$ y $P$ que deben ser tomados en cuenta debido a la degeneración existente en los eigenvalores $m^{2}$ se aplican nuevamente. Así, podemos considerar que cuando $N \rightarrow \infty$, muchas columnas de $\mathbf{F}$ y $\mathbf{G}$ corresponden al mismo valor de $m=m_{q}$ [cf. (5.17)]. Sean $\mathbf{f}$ y $\mathbf{g}$ tales sumas, escritas como

$$
\mathbf{f}=\sum_{E_{n}^{2}-p_{l}^{2}=m_{q}^{2}}[\mathbf{F}]_{q} \quad \mathbf{g}=\sum_{E_{n}^{2}-p_{l}^{2}=m_{q}^{2}}[\mathbf{G}]_{q} .
$$

Así, para energías positivas, las formas límites de $\mathbf{f}$ y $\mathbf{g}$ están dadas por

$$
\psi\left(x_{j}, t_{k}\right)=\frac{1}{2 \pi} \sum_{E=\sqrt{m^{2}+p^{2}}}\left[\cos \left(E t_{k}\right) \cos \left(p x_{j}\right)-(p / E) \sin \left(E t_{k}\right) \sin \left(p x_{j}\right)\right] \Delta E \Delta p,
$$

y

$$
\phi\left(x_{j}, t_{k}\right)=-\frac{i m}{2 \pi} \sum_{E=\sqrt{m^{2}+p^{2}}}(1 / E) \sin \left(E t_{k}\right) \cos \left(p x_{j}\right) \Delta E \Delta p,
$$

respectivamente. Por lo tanto, siguiendo el procedimiento de aproximación de la sección previa, encontramos que estas expresiones convergen a los valores de las integrales

$\psi\left(x_{j}, t_{k}\right)=\frac{1}{2 \pi} \int_{0}^{\infty} d E \int_{-\infty}^{\infty} d p \delta\left(E-\sqrt{m^{2}+p^{2}}\right)\left[\cos \left(E t_{k}\right) \cos \left(p x_{j}\right)-(p / E) \sin \left(E t_{k}\right) \sin \left(p x_{j}\right)\right]$

y

$$
\phi\left(x_{j}, t_{k}\right)=-\frac{i m}{2 \pi} \int_{0}^{\infty} d E \int_{-\infty}^{\infty} d p \delta\left(E-\sqrt{m^{2}+p^{2}}\right)(1 / E) \sin \left(E t_{k}\right) \cos \left(p x_{j}\right),
$$

es decir,

$$
\psi(x, t)=\frac{1}{\pi} \int_{0}^{\infty} \cos \left(t \sqrt{m^{2}+p^{2}}\right) \cos \left(p x_{j}\right) d p-\frac{1}{\pi} \int_{0}^{\infty} \frac{p \sin \left(t \sqrt{m^{2}+p^{2}}\right)}{\sqrt{m^{2}+p^{2}}} \sin (p x) d p
$$




$$
\phi(x, t)=-\frac{i m}{\pi} \int_{0}^{\infty} \frac{\sin \left(t \sqrt{m^{2}+p^{2}}\right)}{\sqrt{m^{2}+p^{2}}} \cos \left(p x_{j}\right) d p
$$

donde hemos eliminado los índices por simplicidad en la notación. Calculando estas dos integrales mediante transformadas de Hankel [23], obtenemos así que las sumas (5.61) y (5.62) convergen a dos de las componentes del propagador continuo ${ }^{3}$

$$
\begin{gathered}
\psi(x, t)= \begin{cases}\frac{m}{2}(-t+x) \frac{J_{1}\left(m \sqrt{t^{2}-x^{2}}\right)}{\sqrt{t^{2}-x^{2}}}, & 0<|x|<t, \\
0, & t<|x|,\end{cases} \\
\phi(x, t)= \begin{cases}-\frac{i m}{2} J_{0}\left(m \sqrt{t^{2}-x^{2}}\right), & 0<|x|<t, \\
0, & t<|x| .\end{cases}
\end{gathered}
$$

Antes de continuar, es importante aclarar qué función de Green estamos obteniendo con la selección (5.58). Desafortunadamente, el estado actual de la técnica que estamos presentando para discretizar y obtener soluciones de la ecuación de Dirac, no permite saber cuál es el tipo de función que obtendremos (si es la causal, la retardada o alguna otra utilizada comúnmente), como se ilustra con los valores dados a las A's y B's al final de este capítulo. Sin embargo, a posteriori, podríamos argüir que el caso de (5.67)-(5.68) corresponde a dos de las componentes espinoriales de la función de Pauli-Jordan, pues si bien $t>0$ en esas ecuaciones, podemos obtener el par correspondiente a $t<0$, tal como se verá enseguida, haciendo que este propagador sea distinto de cero sólo dentro del cono de luz.

Para considerar las amplitudes de las antipartículas debemos realizar las sumas (5.61) y (5.62) sobre la hipérbola inferior $E=-\sqrt{m^{2}+p^{2}}$ (energías negativas), pero haciendo el cambio $t_{k} \rightarrow-t_{k}$, pues así estaremos considerando la contribución al propagador debida a los positrones de energía positiva, ya que estas partículas pueden interpretarse como electrones de energía negativa viajando al pasado. Una simple inspección de (5.61) y (5.62) muestra que el único cambio en este caso, es el signo negativo de (5.61) por un +. Sin embargo, las expresiones resultantes para $\psi$ y $\phi$ no satisfacen la ecuación de Dirac a menos que se intercambien $\psi \rightarrow \phi, \phi \rightarrow \psi$ (esta solución puede obtenerse con una selección adecuada de las matrices A's y B's, tal como lo haremos un poco más adelante en coordenadas nulas). Así tenemos las cuatro componentes del propagador

$$
K(x, t ; 0,0)=\frac{m}{2} \theta(t-|x|)\left(\begin{array}{cc}
(-t+x) J_{1}(m \tau) / \tau & -i J_{0}(m \tau) \\
-i J_{0}(m \tau) & (-t-x) J_{1}(m \tau) / \tau
\end{array}\right)
$$

donde $\theta(x)$ es la función escalón y $\tau=\left(t^{2}-x^{2}\right)^{1 / 2}$. Si la representación de la ecuación de Dirac que se da en [31] se escoge en lugar de la que estamos utilizando, se obtiene

\footnotetext{
${ }^{3}$ Recuérdese que el propagador o función de Green puede ser interpretado como una función de onda [25]. Por otra parte, puede concluírse que (5.67) y (5.68) son efectivamente componentes de una función de Green del hecho de que las sumas (5.60) se realizan sin constantes [a diferencia del caso de Klein-Gordon (5.21), donde los kerneles aparecen de manera explícita].
} 
un cambio de signo en la parte imaginaria y una transposición de las componentes del propagador.

Además, si removemos el factor $-i$ de la segunda matriz en (5.54), obtenemos una solución de la ecuación discreta en la primera representación RI dada en [36]. Por lo tanto, en RI las soluciones son reales e iguales a las de RII (salvo el factor $-i$ de la segunda componente.

Por otra parte, si tomamos la hipérbola derecha o izquierda para realizar las sumas (5.61)(5.62), podemos obtener soluciones taquiónicas de la ecuación de Dirac.

Para mostrar que nuestra solución discreta puede interpretarse en términos de sumas pesadas sobre trayectorias en redes (lattice paths), o en otras palabras, conectarse con el modelo de ajedrez de Feynman del propagador unidimensional, encontramos más conveniente trabajar en las coordenadas nulas $r=(t+x) / 2, s=(t-x) / 2$, en las cuales la ecuación de Dirac discretizada (usando la representación RII) toma la forma

$$
\mathrm{D}_{s} \mathrm{~F}=-i \mathrm{GM}, \quad \mathrm{D}_{r} \mathrm{G}=-i \mathbf{F M} .
$$

Nuevamente, la solución puede escribirse en la forma (5.54) con las substituciones $t \rightarrow s$ y $x \rightarrow r$, junto con $\mathbf{A}_{1}=\mathbf{A}_{2}=\mathbf{A}$ y $\mathbf{B}_{1}=\mathbf{B}_{2}=\mathbf{B}$. La condición sobre $\mathbf{A}$ y $\mathbf{B}$ es ahora

$$
\mathbf{B}=\mathbf{M V ^ { - 1 } \mathbf { A }}
$$

(o $\left.\mathbf{A}=\mathbf{M W}^{-1} \mathbf{B}\right)$, junto con $\mathbf{W V}=\mathbf{M}^{2}$. $\mathbf{W}$ y $\mathbf{V}$ son matrices diagonales con eigenvalores $w_{n}$ y $v_{l}$ correspondiendo a los impulsos discretos asociados a las coordenadas $s$ y $r$ respectivamente. Por lo tanto, un eigenvalor de la masa está dado por

$$
m_{q}^{2}=w_{n} v_{l}, \quad q=l+(n-1) N .
$$

Así, la selección $\mathbf{A}=1, \mathbf{B}=\mathbf{M V}^{-1}$, produce

$$
\mathbf{F}=\left(C_{s} \otimes C_{r}\right)-\left(S_{s} \otimes S_{r}\right), \quad \mathbf{G}=-i\left[\left(S_{s} \otimes C_{r}\right)+\left(C_{s} \otimes S_{r}\right)\right] \mathbf{M V}^{-1} .
$$

La forma límite de $\mathbf{f}=\sum_{q}[\mathbf{F}]_{q}$ y $\mathbf{g}=\sum_{q}[\mathbf{G}]_{q}$, para $m=m_{q}$ y energías positivas, es

$$
\psi(r, s)=\frac{1}{2 \pi} \sum_{w=m^{2} / v} \cos (w s+v r) \varepsilon^{2}, \quad \phi(r, s)=-\frac{i m}{2 \pi} \sum_{w=m^{2} / v}(1 / v) \sin (w s+v r) \varepsilon^{2},
$$

respectivamente (nótese que hemos suprimido los índices). Aquí, $\varepsilon=\Delta w=\Delta v$. Aproximando las sumas de (5.74) por integrales obtenemos

$$
\psi(r, s)=-\frac{m z}{2} \frac{J_{1}(2 m \sigma)}{\sigma}, \quad \phi(r, s)=-\frac{i m}{2} J_{0}(2 m \sigma),
$$

donde $z$ puede ser $r$ o $s$ y $\sigma=(r s)^{1 / 2}, r, s>0$, [cf. (5.67)]. El hecho de que $z$ pueda ser $r$ o $s$, se debe a la relación $w v=m^{2}$ y a la dependencia funcional del integrando correspondiente en $r$ y $s$ (se puede integrar sobre $w$ o sobre $v$ ). Es posible escribir las sumas de (5.74) en una forma más conveniente para nuestro propósito si notamos que 
los ceros de $H_{N}(x)$ son puntos equiespaciados con incremento $\varepsilon$ cuando $N \rightarrow \infty$ [cf. (5.19)] y si usamos algunos resultados del cálculo de diferencias finitas para desarrollar los sumandos de (5.74) en una serie de Newton. Para ilustrar el procedimiento, tomemos la segunda función. Así, de acuerdo a [34],

$$
\sin (s w+r v)=\sum_{n=0}^{\infty} \sum_{m=0}^{\infty}\left(\begin{array}{c}
s \\
n
\end{array}\right)_{\varepsilon}\left(\begin{array}{c}
r \\
m
\end{array}\right)_{\varepsilon} w^{n} v^{m} \sin [(n+m) \pi / 2]
$$

donde $\left(\begin{array}{l}j \\ k\end{array}\right)_{\varepsilon}$ denota el coeficiente binomial generalizado con incremento $\varepsilon$. Puesto que los términos que contribuyen a la suma son aquellos para los cuales $n+m$ es un número impar, podemos escribir $n+m=2 k+1, k=0,1, \cdots$, y $n-m=2 l-1, l$ entero, así que (5.76) toma la forma

$$
\sin (s w+r v)=\sum_{k=0}^{\infty} \sum_{l=-\infty}^{\infty}(-1)^{k}\left(\begin{array}{c}
s \\
k+l
\end{array}\right)_{\varepsilon}\left(\begin{array}{c}
r \\
k-l+1
\end{array}\right)_{\varepsilon} w^{k+l} v^{k-l+1} .
$$

La substitución de este desarrollo en (5.74) da

$$
\phi(r, s)=-i \frac{\pi}{4 N} \sum_{v w=m^{2}} \sum_{k, l}(-1)^{k} m^{2 k+1}\left(\begin{array}{c}
s \\
k+l
\end{array}\right)_{\varepsilon}\left(\begin{array}{c}
r \\
k-l+1
\end{array}\right)_{\varepsilon}(w / v)^{l},
$$

donde hemos usado que $\varepsilon^{2}=\pi^{2} / 2 N$ y la igualdad $w v=m^{2}$. En vista de esta última relación, la suma sobre $l$ en (5.78) produce una serie divergente, a menos que $l=0$. Con $l=0$, la suma sobre $v m=m^{2}$ da $N / 2$ términos (correspondientes a los $N / 2$ ceros positivos de Hermite), haciendo que

$$
\phi(r, s)=\frac{\pi}{8} \sum_{k=0}^{\infty} N_{+-}(-i m \varepsilon)^{2 k+1} .
$$

donde $N_{+-}=C_{k}^{S} C_{k+1}^{R}=\left(\begin{array}{c}S \\ k\end{array}\right)\left(\begin{array}{c}R \\ k+1\end{array}\right)$. Aqí hemos extraído el incremento $\varepsilon$ del coeficiente binomial definiendo los enteros $S$ y $R$ por medio de $s=S \varepsilon$ y $r=R \varepsilon$. De acuerdo al modelo de ajedrez de Feynman del propagador, el entero $N_{+-}$enumera las trayectorias que sigue una partícula relativista moviéndose primero hacia la derecha y hacia la izquierda al final de la trayectoria (ver [36]). En la Figura 3 se da un ejemplo de dos trayectorias: una que comienza y termina yendo hacia la derecha, denotada por trayectoria de tipo $R R$ y la otra, una que comienza yendo hacia la derecha y termina yendo hacia la izquierda, denotada por trayectoria de tipo $R L$. No es difícil ver que una trayectoria de tipo $R L$ involucra $k$ puntos sobre sobre el eje $r$ y $k+1$ sobre el eje $s$, por lo que el número de trayectorias tipo $R L$ que parten del origen y llegan a $(r, s)$ con $k$ puntos en $r$ y $k+1$ en $s$ está dado por $C_{k}^{S} C_{k+1}^{R}$. De igual manera puede verse que el número de trayectorias tipo $R R$ que parten del origen y llegan a $(r, s)$ con $k$ puntos en $r$ y $k$ en $s$ está dado por $C_{k}^{S} C_{k}^{R}$. Por lo tanto, salvo por una constante de normalización (5.79) es la componente no diagonal $K_{+-}$del propagador del electrón cuya forma continua está dada por (5.75). El 
signo de la unidad imaginaria en (5.79) se debe a la representación usada en este cálculo. La simetría en $s$ y $r$ que produce que aparezca $z$ en (5.75) tiene su analogía aquí: $\mathbf{V}$ y $\mathbf{W}$ tienen los mismos elementos diagonales salvo por una permutación. Por lo tanto, podemos substituir $v$ por $w$ en la segunda suma de (5.74) para obtener la componente $K_{-+}$del propagador. Por otro lado, si desarrollamos $\cos (s w+r v)$ siguiendo las mismas líneas, la primera función de (5.74) puede reescribirse en la forma

$$
\psi(r, s)=\frac{\pi}{8} \sum_{k=0}^{\infty} N_{++}(-i m \varepsilon)^{2 k}
$$

donde $N_{++}=\left(\begin{array}{l}S \\ k\end{array}\right)\left(\begin{array}{l}R \\ k\end{array}\right)$ enumera las trayectorias que se mueven a la derecha al principio

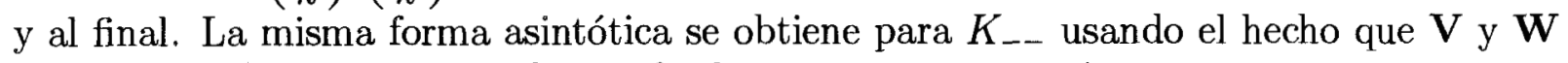
tienen los mismos elementos diagonales hasta una permutación.

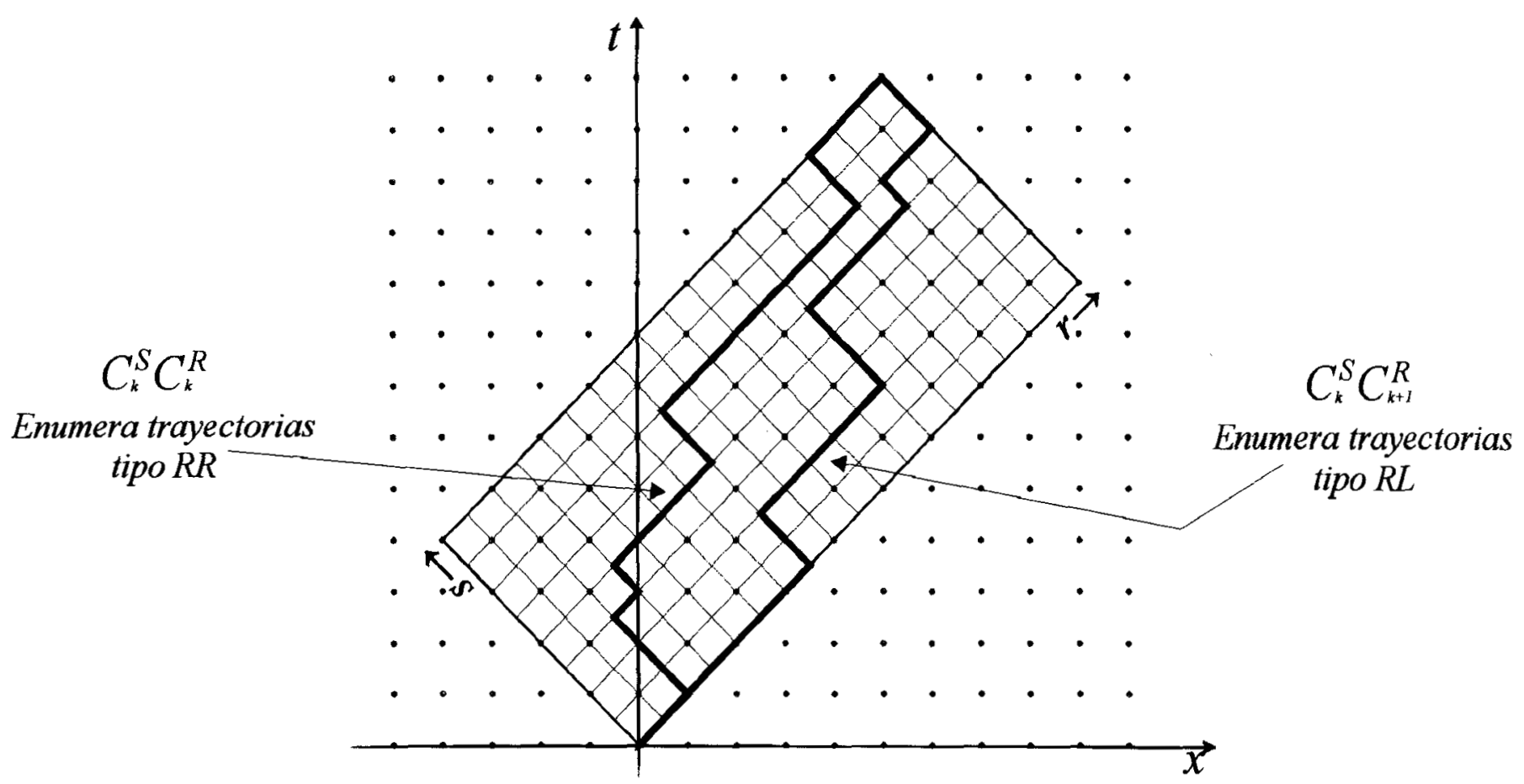

FIGURA 3. Ejemplo de dos trayectorias que comienzan y terminan en puntos coincidentes de una malla uniforme sobre coordenadas nulas $r-s$. Las trayectorias de tipo $R L$ (principio a la derecha, final a la izquierda) están enumeradas por el producto $C_{k}^{S} C_{k+1}^{R}$, mientras que las de tipo $R R$ lo son por $C_{k}^{S} C_{k}^{R}$.

Puesto que la suma sobre $v m=m^{2}$ produce solamente un factor común en (5.79) y (5.80), la forma funcional de estas ecuaciones se debe esencialmente a la parte real e imaginaria 
de la función de onda de la partícula libre exp $[-i(w s+v r)]$. Finalizamos remarcando que este método puede ser usado para obtener más soluciones de la ecuación de Dirac. Como ejemplo final, si escogemos $\mathbf{A}=\mathbf{M} \mathbf{W}^{-1}, \mathbf{B}=1$, se obtiene

$$
\psi(r, s)=-\frac{m}{2} Y_{0}(2 m \sigma), \quad \phi(r, s)=-\frac{m z}{2} \frac{Y_{1}(2 m \sigma)}{\sigma},
$$

donde $Y_{0}(x)$ y $Y_{1}(x)$ son funciones de Bessel de segunda clase. 


\section{Capítulo 6}

\section{Conclusiones}

Los resultados que se han obtenido a lo largo de este trabajo muestran que es posible formular los principios básicos de la Mecánica Cuántica en un espacio lineal de dimensión finita, en contraparte a los espacios de Hilbert normalmente usados, y donde las observables físicas adoptan formas matriciales finitas y los estados de un sistema vienen a ser vectores cuyos elementos son proyecciones de las funciones de onda continuas en ese espacio lineal de dimensión finita. Algunos principios cuánticos, como la forma diferencial de la representación en coordenadas de los impulsos, la interpretación del impulso angular como generador de rotaciones, las relaciones de conmutación, las álgebras asociadas, y las ecuaciones de onda, adoptan en el espacio lineal finito las mismas formas funcionales cuánticas del caso continuo pero con operadores matriciales de dimensión finita (salvo por matrices de proyección en algunos casos) que reproducen o aproximan a los resultados continuos cuando la dimensión del espacio lineal tiende a infinito. Las componentes de los vectores que resuelven las ecuaciones matriciales obtenidas por la proyección de las ecuaciones de onda también reproducen o aproximan a las funciones de onda del sistema que se trate, siempre que éstas se evalúen en ciertos puntos específicos (en una malla o lattice) del espacio-tiempo que son determinados por el tipo de problema en cuestión. Esta última afirmación debe tomarse con cierto cuidado en el caso relativista, pues como se ha mostrado en el último capítulo, los resultados continuos pueden obtenerse a partir de los discretos si en éstos se incluyen muchos más puntos de la malla de energía-impulso de los que realmente corresponden (aunque pueda arguírse, tal como lo hicimos, que esos puntos adicionales satisfacen las condiciones necesarias para añadirlos).

Además de que este esquema de proyección puede usarse como método numérico de solución a problemas cuánticos (como se ha mostrado en el capítulo 4), puede usarse como método de aproximación o de discretización para resolver de forma alternativa algunos sistemas cuánticos, como es el caso de la conexión entre el propagador continuo asociado a la ecuación de Dirac para una partícula libre y su propagador discreto obtenido a través de integrales de trayectoria (sección 5.3), así como el caso no relativista del oscilador para-Bose ([15]). Sin embargo, esta técnica aún no está bien establecida. Basta notar que su aplicación al problema relativista libre en $1+3$ variables no ha sido tratado. Incluso en el caso de $1+1$ variables hay situaciones que aclarar, como la relación de las matrices 
A's y B's de (5.54) y la causalidad del propagador obtenido. Los casos con interacción y su extensión a la Teoría Cuántica de Campos todavía están fuera del alcance del estado actual del método, pero los resultados presentados en este trabajo pueden servir de base para el desarrollo futuro de esta técnica. 


\section{Apéndice A}

\section{Producto de Kronecker}

Si bien el producto tensorial entre matrices (también llamado producto directo o de Kronecker) puede definirse para matrices de distinto rango, la definición que damos enseguida sólo vale para matrices cuadradas de diferente dimensión. Sin embargo, la generalización hacia matrices de distinto rango es inmediata.

Sea $A$ una matriz de $n \times n$ y $B$ una de $m \times m$, ambas definidas sobre los complejos. Entonces su producto directo lo definimos por

$$
A \otimes B=\left(\begin{array}{cccc}
A_{11} B & A_{12} B & \cdots & A_{1 n} B \\
A_{21} B & A_{22} B & \cdots & A_{2 n} B \\
\vdots & \vdots & \ddots & \vdots \\
A_{n 1} B & A_{n 2} B & \cdots & A_{n n} B
\end{array}\right)
$$

La nueva matriz $A \otimes B$ es $n m \times n m$ y como se ve, tiene una partición de $n \times n$ bloques; el bloque $(i, j)$ corresponde a la matriz $A_{i j} B$. Para expresar la componente $(A \otimes B)_{l m}$ en términos de las componentes individuales de las matrices $A$ y $B$, es conveniente primero denotar por $[A]_{i}$ la $i$-ésima columna de $A$. De esta forma una matriz $A$ puede reescribirse como el vector de columnas $A=\left([A]_{1},[A]_{2}, \cdots,[A]_{n}\right)$, de tal forma que las columnas de $A \otimes B$ tienen la forma

$$
\left(A_{1 l}[B]_{j}, A_{2 l}[B]_{j}, \cdots, A_{n l}[B]_{j}\right)^{t}
$$

donde $(\cdot)^{t}$ significa transpuesta y aquí se usa sólo para ahorrar espacio vertical. Así,

$$
[A \otimes B]_{s}=[A]_{l} \otimes[B]_{j}, \quad s=j+(l-1) m
$$

con $j=1,2, \cdots, m$ y $l=1,2, \cdots, n$, y por lo tanto,

$$
(A \otimes B)_{r s}=\left([A]_{l} \otimes[B]_{j}\right)_{r}=A_{k l} B_{i j}, \quad r=i+(k-1) m \quad s=j+(l-1) m,
$$

con $i=1,2, \cdots, m$ y $k=1,2, \cdots, n$. Los índices asociados a la matriz $B$ cambian más rapidamente. En forma similar, el elemento uv del producto

$A \otimes B \otimes C \otimes D$ 
donde $A$ es $n \times n, B$ es $m \times m, C$ es $n^{\prime} \times n^{\prime}$, y $D$ es $m^{\prime} \times m^{\prime}$, puede escribirse como

$$
(A \otimes B \otimes C \otimes D)_{u v}=(A \otimes B)_{r s}(C \otimes D)_{r^{\prime} s^{\prime}}
$$

donde $u=r^{\prime}+(r-1) n^{\prime} m^{\prime}, v=s^{\prime}+(s-1) n^{\prime} m^{\prime}$ y $r^{\prime}, s^{\prime}=1,2, \cdots, n^{\prime} m^{\prime}$ y $r, s=1,2, \cdots, n m$. Continuando el desarrollo,

$$
(A \otimes B \otimes C \otimes D)_{u v}=A_{k l} B_{i j} C_{k^{\prime} l^{\prime}} D_{i^{\prime} j^{\prime}},
$$

con $u=i^{\prime}+\left(k^{\prime}-1\right) m^{\prime}+(i-1) n^{\prime} m^{\prime}+(k-1) n^{\prime} m^{\prime} m$ y $v=j^{\prime}+\left(l^{\prime}-1\right) m^{\prime}+(j-1) n^{\prime} m^{\prime}+(l-$ 1) $n^{\prime} m^{\prime} m$. Los subíndices $i^{\prime}, j^{\prime}, k^{\prime}, l^{\prime}, i, j, k$ y $l$ corren desde 1 hasta el número de entradas de cada una de las matrices correspondientes. Esta propiedad será utilizada más adelante. De las propiedades de este tipo de producto entre matrices, sólo enumeraremos las de más uso en este trabajo y las demostraciones, que no son complicadas, pueden consultarse en las referencias [40] y [41].

Sean las matrices $A, B, C$, y $D$, tales que los productos $A C$ y $B D$ existan; entonces el producto $(A \otimes B)(C \otimes D)$ tiene sentido y

$$
\begin{aligned}
(A \otimes B)(C \otimes D) & =A C \otimes B D, \\
(A \otimes B)^{t} & =A^{t} \otimes B^{t}, \\
(A \otimes B)^{\dagger} & =A^{\dagger} \otimes B^{\dagger} .
\end{aligned}
$$

Si $A$ y $B$ son no singulares entonces $A \otimes B$ es no singular y

$$
(A \otimes B)^{-1}=A^{-1} \otimes B^{-1} .
$$

De (A.8) se puede concluír un corolario importante: un producto tensorial entre dos matrices puede interpretarse como un producto ordinario entre otras dos matrices de mayor dimensión. Con el fin de hacer claro el uso de esta propiedad, tomaremos $A=I_{n}$ y $D=I_{m}$ (el subíndice indica la dimensión de cada matriz). Para poder aplicar la propiedad, las dimensiones de $B$ y $C$ deberán ser $m$ y $n$ respectivamente. De este modo, tendremos que

$$
C_{n} \otimes B_{m}=\left(C_{n} \otimes I_{m}\right)\left(I_{n} \otimes B_{m}\right) .
$$

Es también de interés revisar la estructura de las matrices $\left(I_{n} \otimes B_{m}\right)$ y $\left(C_{n} \otimes I_{m}\right)$. Ya que $I_{n}$ y $I_{m}$ son las matrices identidad de dimensión $n$ y $m$ respectivamente, tenemos que

$$
I_{n} \otimes B_{m}=\left(\begin{array}{cccc}
B_{m} & 0 & \cdots & 0 \\
0 & B_{m} & \cdots & 0 \\
\vdots & \vdots & \ddots & \vdots \\
0 & 0 & \cdots & B_{m}
\end{array}\right)_{n m \times n m}
$$

y

$$
C_{n} \otimes I_{m}=\left(\begin{array}{cccc}
c_{11} I_{m} & c_{12} I_{m} & \cdots & c_{1 n} I_{m} \\
c_{21} I_{m} & c_{22} I_{m} & \cdots & c_{2 n} I_{m} \\
\vdots & \vdots & \ddots & \vdots \\
c_{n 1} I_{m} & c_{n 2} I_{m} & \cdots & c_{n n} I_{m}
\end{array}\right)_{n m \times n m}
$$


y por lo tanto

$$
\left(I_{n} \otimes B_{m}\right)\left(C_{n} \otimes I_{m}\right)=\left(C_{n} \otimes I_{m}\right)\left(I_{n} \otimes B_{m}\right),
$$

es decir, $C_{n} \otimes B_{m}$ puede escribirse como un producto ordinario de matrices que conmutan, aunque el propio producto directo no sea conmutativo en general.

Otra propiedad importante se deriva del siguiente hecho. Un vector $w$ que se construye como el producto tensorial de dos vectores $u$ y $v, u$ de dimensión $n$ y $v$ de dimensión $m$ puede escribirse como

$$
w^{t}=(u \otimes v)^{t}=\left(u_{1} v^{t}, u_{2} v^{t}, \cdots, u_{n} v^{t}\right)
$$

La dimensión de este vector es $n m$ y puede reescribirse en la forma de una matrix $m \times n$, lo cual indicaremos por medio del símbolo $\Leftrightarrow$ en la forma siguiente:

$$
(u \otimes v)=w_{n m} \Leftrightarrow w_{m \times n}=\left(\begin{array}{cccc}
u_{1} v_{1} & v_{1} u_{2} & \cdots & v_{1} u_{n} \\
v_{2} u_{1} & v_{2} u_{2} & \cdots & v_{2} u_{m} \\
\vdots & \vdots & \ddots & \vdots \\
v_{m} u_{1} & v_{m} u_{2} & \cdots & v_{m} u_{n}
\end{array}\right)_{m \times n}
$$

De hecho, cualquier vector de dimensión $N$ puede reescribirse como una matriz de orden $n \times m$ en esa forma si existen $n$ y $m$ tales que $N=n m$. Tomando en cuenta la propiedad (A.12) y esta manera de reescribir vectores tensoriales, no es difícil ver que si $u$ es un vector de dimensión $n m$, y $u_{m \times n}$ es el mismo vector pero reescrito como matriz $m \times n$, entonces

$$
\left(A_{n \times n} \otimes B_{m \times m}\right) u_{n m} \Leftrightarrow B_{m \times m} u_{m \times n} A_{n \times n}^{t} .
$$

Es decir, el producto tensorial de dos matrices, $A$ y $B$, por un vector $u$ en el espacio de dimensión $n m$ puede reescribirse como el producto ordinario de $B$ por $u$ (reescrito en forma matricial) por la transpuesta de $A$. Para mostrar esta propiedad, obsérvese que de acuerdo a (A.12) se tiene $A_{n} \otimes B_{m}=\left(A_{n} \otimes I_{m}\right)\left(I_{n} \otimes B_{m}\right)$. Ahora bien, de acuerdo a la estructura de $\left(A_{n} \otimes I_{m}\right)$, tendremos que para cualquier vector $v$ de dimensión $n m$,

$$
\left(A_{n} \otimes I_{m}\right) v \Leftrightarrow\left(A_{n \times n}\left(v_{m \times n}\right)^{t}\right)^{t}=v_{m \times n} A_{n \times n}^{t},
$$

y la otra parte del producto, el vector $\left(I_{n} \otimes B_{m}\right) u_{n m}$, que jugaría el papel de $v$ en la ecuacón anterior cuando se reescribe en forma matricial, toma la forma $B_{m \times m} u_{m \times n}$.

Este resultado puede reescribirse en una forma más conocida. Sea $u_{n m}^{\prime}=\left(A_{n \times n} \otimes\right.$ $\left.B_{m \times m}\right) u_{n m}$ y sea $u^{\prime}$ su reescritura como matriz, esto es $u^{\prime}=B u A^{t}$ (en donde hemos omitido los subíndices para mayor simplicidad). Tomando la igualdad entre las componentes $(i, j)$ obtenemos

$$
u_{i j}^{\prime}=\sum_{k=1}^{n} \sum_{l=1}^{m} B_{i k} A_{j l} u_{k l} .
$$

Obsérvese que todo vector de $n m$ componentes acepta una reescritura en la forma (A.17), pero cuando dicho vector se forma como un producto tensorial podemos darle otra interpretación que resulta conveniente. Pensemos en dos funciones $f(x)$ y $g(y)$, que toman valores sobre los conjuntos $\left\{x_{1}, x_{2}, \cdots, x_{n}\right\}$ y $\left\{y_{1}, y_{2}, \cdots, y_{m}\right\}$, formando los vectores 
$f=\left(f\left(x_{1}\right), f\left(x_{2}\right), \cdots, f\left(x_{n}\right)\right)$ y $g=\left(g\left(y_{1}\right), g\left(y_{2}\right), \cdots, g\left(y_{m}\right)\right.$. El producto directo $u=g \otimes f$ tiene $n m$ componentes que pueden relacionarse a las componentes individuales de $f$ y $g$ por medio de

$$
u_{r}=f\left(x_{i}\right) g\left(y_{j}\right)
$$

donde

$$
r=1,2, \cdots, n m, \quad r=i+(j-1) n, \quad i=1,2, \cdots, N, \quad j=1,2, \cdots, m .
$$

El índice que corre más rapidamente es el de la coordenada $x$, y como se ve, en este caso la interpretación del vector producto $u$ como una matriz $n \times m$ cuya componente $(i, j)$ es $f\left(x_{i}\right) g\left(y_{j}\right)$, es natural y corresponde al valor de la función de dos variables $u(x, y)=f(x) g(y)$ evaluada en el punto $\left(x_{i}, y_{j}\right)$. Nótese que esta interpretación tiene sentido aún cuando $u(x, y)$ no se pueda escribir como producto de funciones de $x$ y de $y$. En otras palabras, un vector de $n m$ componentes puede interpretarse como la matriz de los $n \times m$ valores que cierta función de dos variables toma en el conjunto de nodos $\left(x_{i}, y_{j}\right)$. Una interpretación similar puede darse para el caso de un número mayor de variables. Tomemos el caso de cuatro variables $x_{1}, x_{2}, x_{3}, x_{4}$, y consideremos dos vectores $f$ y $g$ de $n^{\prime} m^{\prime} n m$ componentes obtenidos a partir de sendas funciones $f\left(x^{1}, x^{2}, x^{3}, x^{4}\right)$ y $g\left(x^{1}, x^{2}, x^{3}, x^{4}\right)$ bajo el ordenamiento (igual para ambos vectores)

$$
f_{v} \equiv f_{j^{\prime} l^{\prime} j l}=f\left(x_{j^{\prime}}^{1}, x_{l^{\prime}}^{2}, x_{j}^{3}, x_{l}^{4}\right) \quad v=j^{\prime}+\left(l^{\prime}-1\right) m^{\prime}+(j-1) n^{\prime} m^{\prime}+(l-1) n^{\prime} m^{\prime} m
$$

donde $j^{\prime}=1,2, \cdots, m^{\prime}, l^{\prime}=1,2, \cdots, n^{\prime}, j=1,2, \cdots, m$, y $l=1,2, \cdots, n$. Entonces el producto $g=(A \otimes B \otimes C \otimes D) f$, puede escribirse en términos de sus componentes como

$$
g_{u}=\sum_{v=1}^{n^{\prime} m^{\prime} n m}(A \otimes B \otimes C \otimes D)_{u v} f_{v}
$$

y utilizando (A.7),

$$
g_{i^{\prime} k^{\prime} i k}=\sum_{j^{\prime}=1}^{m^{\prime}} \sum_{l^{\prime}=1}^{n^{\prime}} \sum_{j=1}^{m} \sum_{l=1}^{n} A_{k l} B_{i j} C_{k^{\prime} l^{\prime}} D_{i^{\prime} j^{\prime}} f_{j^{\prime} l^{\prime} j l}
$$

con $i^{\prime}=1,2, \cdots, m^{\prime}, k^{\prime}=1,2, \cdots, n^{\prime}, i=1,2, \cdots, m$, y $k=1,2, \cdots, n$. 


\section{Apéndice B}

\section{Derivadas parciales discretas}

En este apéndice mostraremos cómo se proyectan las derivadas parciales en espacios polinomiales. Comencemos por el caso de dos variables cartesianas $x$ y $y$ y después generalizaremos estos resultados a un mayor número de ellas.

Sea $\Pi_{(N-1)(M-1)}=\pi_{M-1}(y) \otimes \pi_{N-1}(x)$ el espacio de polinomios tensoriales bivariados, de grado $N-1$ en la variable $x$ y $M-1$ en $y$, que por simplicidad denotaremos por $\Pi$ en este apéndice. Así, si $f \in \Pi$, entonces

$$
f(x, y)=\sum_{j=0}^{N-1} \sum_{k=0}^{M-1} a_{j k} x^{j} y^{k}
$$

Notemos que el espacio de proyección (usado en la sección 2.2) $U_{(N-1)(M-1)}=U_{N-1} \otimes U_{M-1}$ puede escribirse en la forma

$$
U_{(N-1)(M-1)}=e^{-\left(x^{2}+y^{2}\right) / 2} \Pi_{(N-1)(M-1)} .
$$

Usaremos por el momento el espacio de funciones (B.1) en vez de (B.2), por ser ese el espacio donde las derivadas de cualquier orden pueden ser representadas por potencias de matrices con forma de $D_{x}$ [cf. (2.4)]. Después volveremos a usar (B.2) y a representar derivadas en términos de matrices de la forma de $D_{x}^{0}$, aunque esto nos lleve a cuidar el orden de la derivación [ver la discusión sobre esto que está después de la ecuación (2.20)]. Derivando parcialmente (B.1) $n$ veces con respecto a $x, m$ veces con respecto a $y$ y evaluando el resultado en los nodos cartesianos $\left(x_{j}, y_{k}\right)$, obtenemos el vector $\mathbf{F}^{(n, m)}$ de dimensión $N M$ cuyas entradas están dadas por

$$
f_{\alpha}^{(n, m)}=\left.\frac{\partial^{n+m} f(x, y)}{\partial x^{n} \partial y^{m}}\right|_{\left(x_{j}, y_{k}\right)}
$$

donde los índices $\alpha, j \mathrm{y} k$ están relacionados por medio de

$$
\alpha=j+(k-1) N, \quad j=1,2, \cdots, N, \quad k=1,2, \cdots, M,
$$

de tal manera que $\alpha=1,2, \cdots, N M$. Es conocido que una interpolación bivariada única sobre los nodos $\left(x_{j}, y_{k}\right)$ es posible llevarla a cabo en $\Pi$ (ver [20]), así que $f$ puede tomarse 
como una función bivariada no polinomial pero que sea suficientemente diferenciable, y a (B.1) como su polinomio de Taylor asociado. Si escogemos a $j$ (el índice de $x$ ) como el índice de cambio más rápido, y usamos (2.4) tanto para la variable $x$ como para $y$, podemos escribir la proyección de $f^{(n, m)}(x, y)$ como $\mathbf{F}^{(n, m)}$ en téminos de $\mathbf{F}=\mathbf{F}^{(0,0)}$, generalizando $(2.4)$

$$
\mathbf{F}^{(n, m)}=\left(D_{y}{ }^{m} \otimes D_{x}{ }^{n}\right) \mathbf{F}
$$

Esta ecuación es exacta siempre que $f \in \Pi$. Si $f(x, y)$ es una función no polinomial pero derivable, entonces (B.5) deberá contener un término de error que ha sido estimado en [10] para este tipo de casos. Sean $I_{x}$ and $I_{y}$ las matrices de dimensión $N$ y $M$ respectivamente. Definamos las matrices $\mathbf{D}_{x}$ and $\mathbf{D}_{y}$ por medio de

$$
\mathbf{D}_{x}=1_{y} \otimes D_{x}, \quad \mathbf{D}_{y}=D_{y} \otimes 1_{x} .
$$

Por las propiedades del producto de Kronecker, estas matrices conmutan

$$
\mathbf{D}_{x} \mathbf{D}_{y}=\mathbf{D}_{y} \mathbf{D}_{x}=D_{y} \otimes D_{x} .
$$

Más generalmente,

$$
\mathbf{D}_{x}^{n} \mathbf{D}_{y}^{m}=\left(1_{y} \otimes D_{x}^{n}\right)\left(D_{y}^{m} \otimes 1_{x}\right)=D_{y}^{m} \otimes D_{x}^{n}
$$

y por lo tanto, (B.5) toma la forma

$$
\mathbf{F}^{(n, m)}=\mathrm{D}_{x}^{n} \mathrm{D}_{y}^{m} \mathbf{F}
$$

indicando que las derivadas parciales $\partial / \partial x$ y $\partial / \partial y$ adoptan en $\Pi$ las formas tensoriales dadas en (B.6).

Para aplicar estos resultados a una ecuación diferencial parcial y obtener soluciones aproximadas, es necesario conocer la forma que adoptan las funciones coeficientes del operador diferencial a tratar. No es difícil ver que resultan ser matrices diagonales ya que el producto de una función $a(x, y)$ por la derivada parcial de $f(x, y)$ evaluado en los nodos es

$$
a_{r} f_{r}^{(n, m)}=a\left(x_{j}, y_{k}\right) f^{(n, m)}\left(x_{j}, y_{k}\right)
$$

$\left(a\left(x_{j}, y_{k}\right)\right.$ debe estar bien definida en los nodos). Los índices pueden ordenarse de acuerdo a (B.4) produciendo que la función coeficiente $a(x, y)$ pueda representarse por la matriz diagonal con elementos dados por $a_{\alpha}=a\left(x_{j}, y_{k}\right)$, donde $\alpha, j$ y $k$ están relacionados por (B.4). Si denotamos a esta matriz coeficiente por $\mathbf{A}$, la parte del operador diferencial que consiste en el producto $a(x, y) f^{(m, n)}(x, y)$ toma la forma matricial

$$
\mathrm{AD}_{x}^{m} \mathrm{D}_{y}^{n} \mathrm{~F}
$$

Si $a(x, y)$ acepta una expansión de Taylor (esta condición es muy restrictiva y puede no exigirse tanto, pero es adecuada para nuestros propósitos ilustrativos), entonces $\mathbf{A}$ puede definirse por la misma función a través de

$$
\mathbf{A}=a(\mathbf{X}, \mathbf{Y})
$$


donde $\mathbf{X}$ y $\mathbf{Y}$ son las matrices diagonales que representan a las variables $x$ y $y$, y dadas por

$$
\mathbf{X}=I_{y} \otimes X, \quad \mathbf{Y}=Y \otimes I_{x}
$$

donde, como es de esperarse, la matrix $X$ es $N \times N$ y tiene a los nodos $x_{j}, j=1,2 \cdots, N$ a lo largo de su diagonal principal, mientras que $Y$ es $M \times M$ y su diagonal principal contienen a los nodos $y_{y}$.

Si empleamos ahora el espacio de proyección (B.2) no es difícil reescribir estos resultados en términos de las matrices definidas por (2.43) de la sección 2.2 .

La generalización al caso de $q$ variables $x_{1}, x_{2}, \cdots, x_{q}$ es inmediata (ver [12]). Ahora escogeremos $N_{j}$ nodos sobre el eje $x_{j}$ y el espacio de proyección II será seleccionado como el producto tensorial de subespacios de polinomios de grado a lo más $N_{j}-1$ en $x_{j}$, i.e.,

$$
\Pi=\stackrel{q}{j=1}_{j}^{q} \pi_{N_{j}-1} .
$$

Los nodos se ordenarán en tal forma que una función $f\left(x_{1}, \cdots, x_{q}\right)$ evaluada en los nodos produzca el vector $\mathbf{F}$ cuyas entradas sean

$$
f_{r}=f\left(x_{j_{1}}^{1}, x_{j_{2}}^{2}, \cdots, x_{j_{q}}^{q}\right), \quad j_{k}=1,2, \cdots, N_{k}, \quad k=1,2, \cdots, q,
$$

y los índices $r$ y $j_{k}$ estén relacionados por

$$
r=j_{1}+\left(j_{2}-1\right) N_{1}+\left(j_{3}-1\right) N_{1} N_{2} \cdots+\left(j_{q}-1\right) N^{*} / N_{q},
$$

con $j_{k}=1,2, \cdots, N_{k}, k=1,2, \cdots, q$ y donde hemos definido $N^{*}=\prod_{k=1}^{q} N_{k}$. El índice que corre más rapidamente es $j_{1}$, depués $j_{2}$ y así sucesivamente, haciendo que $r=1,2, \cdots, N^{*}$. La representación de $N^{*} \times N^{*}$ de $\partial / \partial x^{k}$, es ahora

$$
\mathbf{D}_{k}=I_{q} \otimes \cdots \otimes I_{k+1} \otimes D_{k} \otimes I_{k-1} \cdots \otimes I_{1},
$$

donde $I_{j}$ es la $N_{j} \times N_{j}$ matriz identidad y $D_{k}$ es una matriz de dimensión $N_{k}$ que tiene la estructura dada por (2.5). Similarmente, la representación de la variable $x^{k}$ es

$$
\mathbf{X}_{k}=I_{q} \otimes \cdots \otimes I_{k+1} \otimes X^{k} \otimes I_{k-1} \cdots \otimes I_{1} .
$$

La forma discreta de las funciones coeficientes de un operador diferencial parcial, se obtienen como matrices diagonales cuyos elementos distintos de cero son los valores de dichas funciones evaluadas en los nodos de acuerdo al orden (B.16). 


\section{Apéndice $\mathbf{C}$}

\section{Convergencia del esquema de proyección}

En este apéndice obtendremos una expresión asintótica para el error que se produce al substituir la derivada de una función dada (evaluada en cierto conjunto de puntos) por el vector obtenido al multiplicar la matriz $\tilde{D}_{x}$ [definida en (2.5) y que en este apéndice denotaremos simplemente como $\tilde{D}$ ] por el vector cuyos elementos son los valores de la función dada en el conjunto de puntos definido de antemano. Es decir, trataremos con más rigor el problema de aproximación definido por nuestro esquema de discretización, y encontraremos una expresión para el término de error $e_{f}$ de la fórmula (2.4)

$$
f^{\prime}=\tilde{D} f+e_{f}
$$

(escrita aquí para $k=1$ ), y mostraremos que este esquema de discretización converge, es decir, el error va a cero cuando $N$, el número de nodos, va a infinito, para un conjunto de funciones bastante amplio, que no necesariamente son lisas.

Comenzaremos por dar algunos resultados ya conocidos (ver [9] y [11]) para funciones totalmente lisas (con derivadas de todo orden) en el intervalo ${ }^{1}(-1,1)$, y cambiaremos ligeramente la notación empleada en 2.1 .

Considérese un conjunto ordenado de $N$ nodos arbitrarios en $(-1,1)$ tal que $-1<x_{1}<$ $x_{2}<\cdots<x_{N}<1$. Sea $L_{N-1}(x)$ el polinomio interpolatorio Lagrangiano de grado $(N-1)$ de una función $f(x)$. Su derivada puede escribirse como

$$
L_{N-1}^{\prime}(x)=\sum_{k=1}^{N} \frac{f\left(x_{k}\right)}{P^{\prime}\left(x_{k}\right)} P_{k}^{\prime}(x)
$$

donde $P_{k}(x)=P(x) /\left(x-x_{k}\right)$ es el $k$-ésimo polinomio fundamental no normalizado de esta interpolación. Consideremos ahora la derivada de $P_{k}(x)$. Sumando y restando la

${ }^{1}$ El hecho de seleccionar $(-1,1)$ como el dominio de funciones, no quita generalidad a los resultados, pues una trasformación lineal lleva $(-1,1) \rightarrow(a, b)$, con $a$ y $b$ arbitrarios 
cantidad $P_{k}(x) \sum_{\substack{l=1 \\ l \neq k}}^{N}\left[1 /\left(x_{k}-x_{l}\right)\right]$ a $P_{k}^{\prime}(x)$ obtenemos

$$
P_{k}^{\prime}(x)=\sum_{\substack{l=1 \\ l \neq k}}^{N} \frac{P_{l}(x)}{\left(x_{l}-x_{k}\right)}+P_{k}(x) \sum_{\substack{l=1 \\ l \neq k}}^{N} \frac{1}{\left(x_{k}-x_{l}\right)}=\sum_{l=1}^{N} P_{l}(x) D_{l k}
$$

donde $D_{l k}, l, k=1,2, \cdots, N$ son los elementos de la matriz $D$, definida como $D_{x}$ en (2.5). Así,

$$
L_{N-1}^{\prime}(x)=\sum_{l, k=1}^{N} P_{l}(x) D_{l k} \frac{f\left(x_{k}\right)}{P^{\prime}\left(x_{k}\right)}
$$

Evaluando esta derivada en los nodos, se obtiene la ecuación matricial $L_{N-1}^{\prime}=P D P^{-1} f=$ $\tilde{D} f$, donde $L_{N-1}^{\prime}$ y $f$ denotan vectores de $R^{N}$ con componentes $L_{N-1}^{\prime}\left(x_{j}\right)$ y $f\left(x_{j}\right), j=$ $1,2 \cdots, N$, respectivamente, y $P$ es la matriz definida como $Q$ en (2.5). Las derivadas de mayor orden se manejan en la misma forma y resultan en potencias de la matriz $\tilde{D}$ del mismo orden. Así, definiendo el vector de residuo o error $E(f, N, k) \equiv E$ por medio de

$$
E_{j}(f, N, k)=f^{(k)}\left(x_{j}\right)-L_{N-1}^{(k)}\left(x_{j}\right), \quad j=1,2 \cdots, N, \quad k=0,1, \cdots,
$$

llegamos a la ecuación (2.4), con la notación ligeramente distinta. En esta expresión se supone que $f$ es suficientemente diferenciable. Nótese que si $f$ es un polinomio de grado $N-1$ a lo más, $E_{j}(f, N, k)=0$, y la interpolación de $L_{N-1}^{(k)}$ da $f^{(k)}(x)$ exactamente. Por otra parte, si $f \in C^{N-1}[-1,1]$ y $f^{(N)}$ existe en $(-1,1)$, entonces vale el residuo de tipo Cauchy (ver [9]):

$$
E_{j}(f, N, k)=\frac{1}{N !} f^{(N)}\left(\xi_{j}\right) P^{(k)}\left(x_{j}\right) \quad j=1,2 \cdots, N, \quad k=1, \cdots, N,
$$

donde $\xi_{j}$ es un punto de $\left(x_{1}, x_{N}\right)$ dependiente de $k$ y $N$.

Ahora calcularemos una fórmula asintótica para el error para cierta clase de funciones no lisas. Encontraremos que el uso de ceros de polinomios de Jacobi como nodos en este esquema de diferenciación conduce a aproximantes precisos y convergentes.

Sean $\alpha$ y $\beta$ parámetros reales, $\alpha, \beta>-1$, y $\gamma(x)$ cierta función relacionada a la función peso $\omega(x)$ de los polinomios de Jacobi $P_{N}^{(\alpha, \beta)}(x)$ dadas por

$$
\gamma(x)=(1-x)^{\frac{\alpha+1}{2}}(1+x)^{\frac{\beta+1}{2}} \quad \omega(x)=(1-x)^{\alpha}(1+x)^{\beta} .
$$

Sea $W^{1}[-1,1]$ el espacio de Sóbolev de todas las funciones reales $f$ absolutamente continuas en $[-1,1]$ tales que su derivada $f^{\prime} \in L_{2}[-1,1]$. Tomemos $f \in W^{1}[-1,1]$ y definamos el núcleo

$$
F(x, y)= \begin{cases}\frac{f(x)-f(y)}{x-y}, & x \neq y, \\ f^{\prime}(y), & x=y .\end{cases}
$$

Entonces podemos reescribir la componente $j$-ésima del vector residuo como

$$
E_{j}=P_{N}^{\prime}\left(x_{j}\right) \sum_{k=1}^{N} \frac{F\left(x_{j}, x_{k}\right)}{P_{N}^{\prime}\left(x_{k}\right)} \quad j=1,2 \cdots, N
$$


Esta suma puede ser aproximada por cierta integral como se muestra enseguida. Considérese la integral

$$
\int_{-1}^{1} \gamma^{2}(x) P_{N}^{(\alpha, \beta) \prime}(x) F\left(x_{j}, x\right) d x=\int_{-1}^{1} \omega(x)\left(1-x^{2}\right) P_{N}^{(\alpha, \beta) \prime}(x) F\left(x_{j}, x\right) d x .
$$

Si $N, \alpha$ y $\beta$ están fijos y $f \in W^{1}[-1,1]$, esta integral existe y puede entonces desarrollarse en una cuadratura de tipo Gauss-Jacobi [49]

$$
\int_{-1}^{1} \omega(x)\left(1-x^{2}\right) P_{N}^{(\alpha, \beta) \prime}(x) F\left(x_{j}, x\right) d x=\lim _{M \rightarrow \infty} \sum_{k=1}^{M} \lambda_{k}\left(1-y_{k}^{2}\right) P_{N}^{(\alpha, \beta) \prime}\left(y_{k}\right) F\left(x_{j}, y_{k}\right)
$$

donde $y_{1}, y_{2}, \cdots, y_{M}$ son los ceros del polinomio de Jacobi $P_{M}^{(\alpha, \beta)}(x)$. Haciendo uso de las expresiones para los números de Christoffel [49]

$$
\lambda_{k}=\frac{C_{M}^{(\alpha, \beta)}}{\left(1-y_{k}^{2}\right)\left[P_{M}^{(\alpha, \beta) \prime}\left(y_{k}\right)\right]^{2}}, \quad C_{M}^{(\alpha, \beta)}=2^{\alpha+\beta+1} \frac{\Gamma(M+\alpha+1) \Gamma(M+\beta+1)}{\Gamma(M+1) \Gamma(M+\alpha+\beta+1)},
$$

donde $k=1,2, \cdots, M$ y $\Gamma(x)$ es la función gamma, se tiene

$$
\int_{-1}^{1} \omega(x)\left(1-x^{2}\right) P_{N}^{(\alpha, \beta) \prime}(x) F\left(x_{j}, x\right) d x=\lim _{M \rightarrow \infty} C_{M}^{(\alpha, \beta)} \sum_{k=1}^{M} \frac{P_{N}^{(\alpha, \beta) \prime}\left(y_{k}\right) F\left(x_{j}, y_{k}\right)}{\left[P_{M}^{(\alpha, \beta) \prime}\left(y_{k}\right)\right]^{2}}
$$

Ahora, si $x_{1}, x_{2}, \cdots, x_{N}$ son los ceros de $P_{M}^{(\alpha, \beta)}(x)$ y $N$ es suficientemente grande,

$$
\int_{-1}^{1} \omega(x)\left(1-x^{2}\right) P_{N}^{(\alpha, \beta) \prime}(x) F\left(x_{j}, x\right) d x \approx C_{N}^{(\alpha, \beta)} \sum_{k=1}^{N} \frac{F\left(x_{j}, y_{k}\right)}{P_{N}^{(\alpha, \beta) \prime}\left(x_{k}\right)}, \quad N \rightarrow \infty .
$$

Por lo tanto, usando (C.9) obtenemos que

$$
E_{j} \approx \frac{P_{N}^{(\alpha, \beta) \prime}\left(x_{j}\right)}{C_{N}^{(\alpha, \beta)}} \int_{-1}^{1} \gamma^{2}(x) P_{N}^{(\alpha, \beta) \prime}(x) F\left(x_{j}, x\right) d x, \quad j=1,2, \cdots, N, \quad N \rightarrow \infty
$$

Definamos ahora una función continua de variable $t \in[-1,1]$ a través de

$$
G(x, t)=\int_{x_{j}}^{t} F(x, y) d y, \quad x \in(-1,1) .
$$

Entonces, una integración por partes produce

$$
E_{j} \approx-\frac{P_{N}^{(\alpha, \beta) \prime}\left(x_{j}\right)}{C_{N}^{(\alpha, \beta)}} \int_{-1}^{1} G\left(x_{j}, x\right)\left[\gamma^{2}(x) P_{N}^{(\alpha, \beta) \prime}(x)\right]^{\prime} d x
$$

Usando ahora la ecuación diferencial de los polinomios de Jacobi obtenemos

$$
E_{j} \approx K P_{N}^{(\alpha, \beta) \prime}\left(x_{j}\right) \times \int_{-1}^{1} \omega(x) P_{N}^{(\alpha, \beta)}(x) G\left(x_{j}, x\right) d x, \quad j=1,2, \cdots, N, \quad N \rightarrow \infty
$$


donde

$$
K=\frac{N(N+\alpha+\beta+1)}{C_{N}^{(\alpha, \beta)}} .
$$

La integral de (C.18) está relacionada directamente al $N$-ésimo coeficiente de la serie de Fourier convergente

$$
G\left(x_{j}, x\right)=\sum_{l=0}^{\infty} g_{j l} P_{l}^{(\alpha, \beta)}(x), \quad j=1,2, \cdots, N
$$

a través de

$$
g_{j l}=\frac{(2 N+\alpha+\beta+1)}{C_{N}^{(\alpha, \beta)}} \int_{-1}^{1} \omega(x) P_{N}^{(\alpha, \beta)}(x) G\left(x_{j}, x\right) d x .
$$

Por lo tanto, la substitución de esta igualdad en (C.18) produce

$$
E_{j} \approx \frac{N(N+\alpha+\beta+1)}{(2 N+\alpha+\beta+1)} g_{j N} P_{N}^{(\alpha, \beta) \prime}\left(x_{j}\right) \approx N g_{j N} P_{N}^{(\alpha, \beta) \prime}\left(x_{j}\right)
$$

para $j=1,2, \cdots, N, \quad N \rightarrow \infty$.

Ya que la integral $\int_{-1}^{1} \omega(x)\left[G\left(x_{j}, x\right)\right]^{2} d x$ existe y es finita, la serie de Fourier de $G\left(x_{j}, x\right)$ converge en espacio pesado de funciones $L_{2}[-1,1 ; \omega]$ y por lo tanto, $g_{j N} \rightarrow 0$ cuando $N \rightarrow \infty$. Esto muestra la convergencia de este esquema para una clase bastante amplia de funciones. Así, tenemos el siguiente

Teorema. Sea $f \in W^{1}[-1,1]$ y sean $x_{1}, x_{2}, \cdots x_{N}$ los $N$ ceros del polinomio de Jacobi $P_{N}^{(\alpha, \beta)}(x)$. Sea también

$$
E(x)=f^{\prime}(x)-L_{N-1}^{\prime}(x), x \in(-1,1)
$$

el residuo entre la derivada de $f$ y la del polinomio interpolatorio de Lagrange que coincide con $f$ en los ceros de $P_{N}^{(\alpha, ß)}(x)$. Entonces, la expresión asintótica de $E(x)$ en $x_{j}, j=1,2, \cdots, N$ cuando $N \rightarrow \infty$ está dada por (C.22), donde $g_{j N}$ es el $N$-ésimo coeficiente de Fourier de la función $G\left(x_{j}, x\right)$ dado por (C.21).

De acuerdo a este resultado, el esquema de derivación discreta presentado en este trabajo converge en los nodos a los valores exactos de la derivada de una función $f$, si los coeficientes de la serie de Fourier de la función $G\left(x_{j}, x\right)$, definida en términos de $f$ [ver (C.8) y (C.16)], decaen a cero rápidamente.

Es posible precisar más este resultado si se define más a la función $f$. Supongamos que $f$ es cóncava (o convexa) en $[-1,1]$. Entonces el cociente $(f(y)-f(x)) /(y-x)$ está entre $f^{\prime}(y)$ y $f^{\prime}(x)$, por lo que

$$
\left[f(x)-f\left(x_{j}\right)\right] \leq G\left(x_{j}, x\right) \leq\left(x-x_{j}\right) f^{\prime}\left(x_{j}\right), \quad x \in[-1,1] .
$$

Si $f$ es convexa las desigualdades se invierten. En cualquier caso tenemos que

$$
\left[G\left(x_{j}, x\right)\right]^{2} \leq\left[f(x)-f\left(x_{j}\right)\right]^{2}+\left[\left(x-x_{j}\right) f^{\prime}\left(x_{j}\right)\right]^{2}, \quad x \in[-1,1],
$$


y así, las normas cuadradas de $G\left(x_{j}, x\right), f(x)-f\left(x_{j}\right)$ y $\left(x-x_{j}\right) f^{\prime}\left(x_{j}\right)$ en $L_{2}[-1,1 ; \omega]$, satisfacen desigualdades similares. Por lo tanto, la fórmula de Parseval produce

$$
\sum_{l=0}^{\infty} g_{j l}^{2} \leq \sum_{l=0}^{\infty} f_{j l}^{2}+\sum_{l=0}^{1} q_{j l}^{2}
$$

donde $q_{j 0}$ y $q_{j 1}$ son los dos coeficientes de la expansión de Fourier en términos de los polinomios de Jacobi de la función lineal $\left(x-x_{j}\right) f^{\prime}\left(x_{j}\right)$ y los valores $f_{j l}$ son los coeficientes que corresponden a $f(x)-f\left(x_{j}\right)$. Por lo tanto, $\left|g_{j N}\right| \approx\left|f_{j N}\right|, N \rightarrow \infty$. Ya que $f\left(x_{j}\right)$ es una constante, los términos de orden grande en la expansión de $f(x)$ y $f(x)-f\left(x_{j}\right)$ coinciden, por lo que

$$
\left|g_{j N}\right| \approx\left|f_{j N}\right|=\left|f_{N}\right|, \quad N \rightarrow \infty,
$$

donde $f_{N}$ denota el $N$-ésimo coeficiente de la expansión de Jacobi

$$
f(x)=\sum_{l=0}^{\infty} f_{l} P_{l}^{(\alpha, \beta)}(x)
$$

dado por

$$
f_{N}=\frac{(2 N+\alpha+\beta+1)}{C_{N}^{(\alpha, \beta)}} \int_{-1}^{1} \omega(x) P_{N}^{(\alpha, \beta)}(x) f(x) d x .
$$

Así, la substitución de (C.27) en (C.22) produce

$$
\left|E_{j}\right| \approx N\left|f_{N} P_{N}^{(\alpha, \beta) \prime}\left(x_{j}\right)\right| \quad j=1,2, \cdots, N, \quad N \rightarrow \infty .
$$

Nótese el parecido de esta expresión con (C.6): ambos son proporcionales a $P_{N}^{(\alpha, \beta) \prime}\left(x_{j}\right)$ y al coeficiente $N$-ésimo de la expansión de $f$ en la base correspondiente. Es posible simplificar más (C.30) si tomamos en cuenta la estimación (ver [49])

$$
P_{N}^{(\alpha, \beta) \prime}(x)=O\left(N^{q}\right), \quad q=\max (\alpha+2, \beta+2),
$$

válida uniformemente en $x, x \in[-1,1]$. Con el objeto de dar una expresión para (C.30) que dependa sólo de $N$, tomaremos un ejemplo.

Considérese la función convexa

$$
f(x)=(1+x)^{s}, \quad s>0 .
$$

La expansión de Fourier de esta función en términos de polinomios de Jacobi está dada por [22]:

$$
(1+x)^{s}=2^{s} \sum_{n=0}^{\infty} f_{n} P_{N}^{(\alpha, \beta)}(x)
$$

con los coeficientes dados por

$$
f_{n}=(-1)^{n} \frac{(2 n+\alpha+\beta+1) \Gamma(n+\alpha+\beta+1) \Gamma(\beta+s+1)(-s)_{n}}{\Gamma(n+\beta+1) \Gamma(n+\alpha+\beta+s+2)}
$$


donde $(a)_{n}=\Gamma(a+n) / \Gamma(a)$ es el símbolo de Pochhammer. Para simplificar, tomemos $\alpha=\beta=0$ y escribamos la potencia $s$ como $s=m+\rho$, donde $0 \leq \rho<1$ y $m$ es la parte entera de $s$. Así, fijando $s$, una estimación asintótica produce $\left|f_{N}\right| \approx K(m, \rho) / N^{2 m+2 \rho+1}$, donde la constante $K(m, \rho)$ no depende de $N$. Por lo tanto,

$$
f_{n}=O\left(N^{-2 s-1}\right) .
$$

Finalmente, substituyendo esta expresión y (C.31) (con $\alpha=\beta=0$ ) en (C.30), obtenemos la norma (máxima) del vector de error que nuestro esquema de derivación produce para (C.32)

$$
\|E\|=O\left(N^{-2(s-1)}\right),
$$

lo que muestra convergencia para $s \geq 1$ [en el caso $s=1$ el esquema de derivación produce resultados exactos porque entonces (C.32) es un polinomio. 


\section{Bibliografía}

[1] M. Abramowitz and I.A. Stegun (eds.), Handbook of Mathematical Functions (Dover Publications, New York, 1972)

[2] R. Arshansky and L.P. Horwitz, The quantum relativistic two-body bound state. I. The spectrum, J. Math. Phys. 30 (1989) 66

[3] P.G. Bergmann and J.H.Brunning, On the connection between Phase Shifts and Scattering Potential, Rev. Mod. Phys. 21 (1949) 480

[4] N.N. Bogoliubov, y D.V. Shirkov, Intoduction to the theory of quantized fields, Intersc. Publ. Inc., New York (1959)

[5] N.N. Bogoliubov, A.A. Logunov y I.T. Todorov, Introduction to axiomatic quantum field theory, Benjamin/Cummings Publ. Co., Massachusetts (1978), second printing.

[6] L. Bracci and G. Fiorentini, Bounds on a hypothetical fundamental length, IFUP TH-83/18 Pisa, Italy (1983)

[7] J.C. Breckenridge, V. Elias and T.G. Steele, Massless scalar field theory in a quantized space-time, preprint 9501108, Los Alamos Preprint Server.

[8] M. Bruschi, R.G. Campos and E. Pace, On a method for computing eigenvalues and eigenfunctions of linear differential operators, Il Nuovo Cimento 105 B (1990) pp. 131-163.

[9] F. Calogero, Lagrangian interpolation and differentiation, Lett. Nuovo Cimento 35, 273 (1982).

[10] F. Calogero, Interpolation, differentiation and solution of eigenvalue problems in more than one dimension, Lett. Nuovo Cimento 38 (1983) pp. 453-459

[11] F. Calogero, Interpolation, differentiation and solution of eigenvalue problems for periodic functions, Lett. Nuovo Cimento 39, 305 (1984).

[12] F. Calogero, Interpolation in multidimensions, a convenient finite-dimensional matrix representation of the (partial) differential operators, and some applications, J. Math. Phys. 34, 4704 (1993) 
[13] R.G. Campos, A nonlocal equation for the wave function of the harmonic oscillator when the position spectrum is.to be made discrete, Rev. Mex. Fis., 29, 217-236 (1983)

[14] R.G. Campos, A non-perturbative method for the $\alpha x^{2}+\beta x^{4}$ interaction, Rev. Mex. Fis., 32, 379-400 (1986)

[15] R.G. Campos, The para-Bose oscillator in a finite linear space, Il Nuovo Cimento $100 \mathrm{~B}, 485-492$ (1987)

[16] R.G. Campos y R. Muñoz B., On Certain Numerical Technique to Solve SturmLiouville Systems (I), Rev. Mex. Fís. 36 (1990) pp. 1-5.

[17] R.G. Campos and L. Juárez Z, A discretization of the continuous Fourier transform, Il Nuovo Cimento 107 B, 703-711 (1992)

[18] R.G. Campos, A quadrature formula for the Hankel transform, Numer. Alg., 9, 343354 (1995)

[19] R.G. Campos, Solving singular nonlinear two point boundary value problems, Bol. Soc. Mat. Mex., 3, 279 (1997)

[20] E.W. Cheney, Multivariate approximation theory: selected topics, SIAM Publications, Philadelphia, Pennsylvania, (1986)

[21] P.A.M. Dirac, , Canadian J. Math. 2 (1950) 129

[22] A. Erdélyi (Editor), Higher Transcendental Functions, Vol. II, McGraw-Hill Book Co., Inc., New York (1954)

[23] A. Erdélyi (Editor), Tables of Integral Transforms, Vols.I, II, McGraw-Hill Book Co., Inc., New York (1954)

[24] G. Feinberg, Possibility of faster-than-ligth particles, Phys. Rev. 159 (1967) 1089

[25] R.P. Feynman and A.R. Hibbs, Quantum Mechanics and path integrals, McGraw-Hill Inc., New York, (1965)

[26] K.W. Ford, Electrodynamics with a quantum of length, Phys. Rev. 175 (1968) 20482053

[27] R. Friedberg and T.D. Lee, Discrete Quantum Mechanics, Nucl. Phys. B225 (1983) $1-52$

[28] L. Garay, Quantum gravity and minimum length, Int. J. Mod. Phys. A10 (1995) $145-166$

[29] Phil Gibbs, The Small Scale Structure of Space-Time: A Bibliographical Review, HEP-TH/9506171 
[30] I.S. Gradshteyn and I.M. Ryzhik, Table of Integrals, Series, and Products, (fifth ed) Academic Press, Inc., London (1994)

[31] T. Jacobson and L.S. Schulman, Quantum stochastics: the passage from a relativistic to a non-relativistic path integral, J. Phys. A 17 375-383 (1984)

[32] E.M. de Jager, The Lorentz invariant solutions of the Klein-Gordon equation, I, II y III, Indag. Math. 25, pp 515-558 (1963)

[33] A. Jannussis, Small-distance derivative model in relativistic mechanics, Hadronic J. 10 (1987) 79-82

[34] Ch. Jordan, Calculus of finite differences, Chelsea Publishing Co., New York, (1960)

[35] V.A. Karmanov, On the derivation of the electron propagator from a random walk, Phys. Lett. A 174 371-376 (1993)

[36] L.H. Kauffman and H.P. Noyes, Discrete Physics and the Dirac equation, Phys. Lett. A 218 139-146 (1996)

[37] Y.P. Kravchenko, M.A. Liberman and B. Johansson, Exact solution for a hydrogen atom in a magnetic field of arbitrary strength, Phys. Rev. A, 54, 287 (1996).

[38] N.N. Lebedev, I.P. Skalskaya y Y.S. Uflyand, Worked problems in applied mathematics, Dover Publications Inc., New York (1965)

[39] T.D. Lee, Can time be a discrete dynamical variable?, Phys. Lett. 122B (1983) 21722

[40] Charles Van Loan, Computational Frameworks for the Fast Fourier Transform, SIAM Publications, Frontiers in Applied Mathematics, Philadelphia (1992)

[41] M. Marcus and H. Minc, A survey of matrix theory and matrix inequalities, Dover Publications, Inc., New York (1992)

[42] N. Mukunda, C.G. Sudarshan, J.K. Sharma and G.L. Metha, Representations and properties of para-Bose oscillator operators I. Energy position and momentum eigenstates, J. Math. Phys. 21 (1980) 2386-2394

[43] Y. Ohnuki and S. Kamefuchi, On the wave-mechanical representation of a Bose-like oscillator, J. Math. Phys. 19 (1978) 67-78

[44] W. Pauli, Comunicación privada a Snyder, ([Sny47]).

[45] F.A.E. Pirani and Schild, On the quantization of Einstein's Gravitational Field Equations, Phys. Rev. 79 (1950) 986

[46] T. Popoviciu, Sur certains problèmes de maximum de Stieltjes, Bull. Math. Soc. Roumaine Sci. 38, (1936) 73. 
[47] G. Prugovecki, Principles of Quantum General Relativity, World Scientific (1995)

[48] H.S. Snyder, Quantized space-time, Phys. Rev., 71 (1947) p. 38.

[49] G. Szegö, Orthogonal Polynomials, 4th ed., American Mathematical Society, Providence, RI 1976.

[50] A.F. Timan, Theory of Approximation of functions of a real variable, Dover Publications, Inc., New York (1994).

[51] I. Watanabe, On the quantization of physical space-time operators, Prog. Theor. Phys., 24 (1960) pp. 465-483

[52] J. Wess, Quantum groups and q-lattices in phase space, Lecture given at the 5th Hellenic School and Workshop on Elementary Particle Physics, CORFU (1995)

[53] E.P. Wigner, Do the Equations of Motion determine the Quantum Mechanical Relations?, Phys. Rew. 77 (1950) 711-712

[54] B.S. de Witt and C.M. De Witt, The Quantum Theory of Interacting Gravitational and Spinor Fields, Phys. Rev. 79 (1952) 116

[55] H. Yukawa, Quantum Theory of Non-Local Fields. Part I. Free Fields, Phys. Rev. 77 (1950) 219 